\title{
Physicochemical modulation of huntingtin aggregation on lipid membranes: Implications for Huntington's disease
}

Maxmore Chaibva

Follow this and additional works at: https://researchrepository.wvu.edu/etd

\section{Recommended Citation}

Chaibva, Maxmore, "Physicochemical modulation of huntingtin aggregation on lipid membranes: Implications for Huntington's disease" (2016). Graduate Theses, Dissertations, and Problem Reports. 5332.

https://researchrepository.wvu.edu/etd/5332

This Dissertation is protected by copyright and/or related rights. It has been brought to you by the The Research Repository @ WVU with permission from the rights-holder(s). You are free to use this Dissertation in any way that is permitted by the copyright and related rights legislation that applies to your use. For other uses you must obtain permission from the rights-holder(s) directly, unless additional rights are indicated by a Creative Commons license in the record and/ or on the work itself. This Dissertation has been accepted for inclusion in WVU Graduate Theses, Dissertations, and Problem Reports collection by an authorized administrator of The Research Repository @ WVU.

For more information, please contact researchrepository@mail.wvu.edu. 
Physicochemical modulation of huntingtin aggregation on lipid membranes:

Implications for Huntington's disease

\title{
Maxmore Chaibva
}

\author{
Dissertation submitted \\ to the Eberly College of Arts and Sciences \\ at West Virginia University
}

in partial fulfillment of the requirements for the degree of

Doctor of Philosophy in

Physical Chemistry

\author{
Justin Legleiter, Ph.D., Chair \\ Terry Gullion, Ph.D. \\ Fabien Goulay, Ph.D. \\ Visvanathan Ramamurthy, Ph.D. \\ Blake Mertz, Ph.D. \\ C. Eugene Bennett Department of Chemistry \\ Morgantown, West Virginia \\ 2016
}

Keywords: atomic force microscopy, scanning probe acceleration microscopy, mechanical properties, lipid bilayers , protein aggregation, acetylation, membrane curvature

\section{Copyright 2016 Maxmore Chaibva}




\begin{abstract}
Physicochemical modulation of huntingtin aggregation on lipid membranes: Implications for Huntington's disease
\end{abstract}

\title{
Maxmore Chaibva
}

Huntington disease is a genetic, neurodegenerative disease caused by an expanded polyglutamine (polyQ) in the first exon of the huntingtin (htt) protein, facilitating its aggregation, leading to the formation of a diverse population of potentially toxic aggregate species, such as oligomers, fibrils, and annular aggregates. Htt interacts with a variety of membranous structures within the cell, and the first seventeen amino acids $\left(\mathrm{Nt}^{17}\right)$ of htt directly flanking the polyQ domain is an amphipathic $\alpha$-helix (AH) lipid-binding domain. AHs are also known to detect membrane curvature. $\mathrm{Nt}^{17}$ also promotes diverse aggregate species of htt and undergoes a number of posttranslational modifications that can modulate htt's toxicity, subcellular localization, and trafficking of vesicles. To get in-depth mechanistic insights of huntingtin aggregation, both chemical and physical modulators of the aggregation process were explored. Specifically, the importance of htt acetylation and the role of membrane curvature on htt aggregation were investigated using atomic force microscopy (AFM), which has become a robust technique to obtain physical insights into the formation of toxic protein aggregates associated with amyloid diseases on lipid membranes. Acetylation of htt exon 1, and synthetic truncated htt exon1 mimicking peptide $\left(\mathrm{Nt}^{17} \mathrm{Q}_{35} \mathrm{P}_{10} \mathrm{KK}\right)$ was achieved using a selective covalent label sulfo-Nhydroxysuccinimide (NHSA) in molar ratios of $1 \mathrm{x}, 2 \mathrm{x}$, and $3 \mathrm{x}$ NHSA per peptide. Htt acetylation was found to decrease fibril formation in solution and promoted the formation of larger globular aggregates. Acetylation strongly altered htt's ability to bind lipid membranes. 
However, one of the several limitations associated with using these current flat, supported bilayers as model surfaces is the absence of membrane curvature, which can heavily influence the interaction of proteins at lipid interfaces. Using an AFM force reconstruction technique, silicon substrate, and silica nanobeads, model lipid bilayer system was developed and validated in which the underlying solid support is comprised of flat and curved regions to induce regions of curvature in the bilayer. This model bilayer system was exposed to $\mathrm{Nt}^{17} \mathrm{Q}_{35} \mathrm{P}_{10} \mathrm{KK}$ peptide, and this peptide preferentially bound and accumulated to curved membranes, consistent with the ability of AHs to sense membrane curvature. 


\section{Dedication}

I dedicate this Ph.D. dissertation to my wife, Faith Ruzvidzo Chaibva, my two boys Donnel Tavonga , and Shiloh Nenyasha Chaibva. You were my inspiration.

Faith, thank you for your unconditional support, prayers, and for taking care of the kids during these five years. You always stood by me. You left your professional and came to USA with me. You were a stay home mum for 5 years while I was studying. You are awesome.

"Whatever you vividly imagine, ardently desire, sincerely believe and enthusiastically act upon must inevitably come to pass."

- $\underline{\text { Paul J. Meyer }}$ 


\section{Acknowledgements}

My deepest gratitude goes to my Ph.D advisor, Dr. Justin Legleiter for his support, encouragement, and scientific freedom during my graduate studies in his research lab. He gave me invaluable opportunities to troubleshoot technical and experimental questions that arose during my experiments, as well as opportunities for developing lab protocols. He also presented me with challenges and opportunities to write research reviews and book chapters so that I get credit which would otherwise have gone to him alone. If I am given a second chance to choose a Ph.D advisor, I would still go with Justin Legleiter. I am truly grateful for his patience, motivation, and mentorship.

I would like to thank West Virginia University, C. Eugene Bennett Department of Chemistry for providing me with a doctoral scholarship, and the additional funding agencies, including NIH, WVU NanoSafe who funded this work. I would also like to thank my committee members; particularly Dr. Visvanathan Ramamurthy and his graduate student, Abigail Hayes, for their help and guidance in reconstructing the new huntingtin protein constructs to fix the mutation on protein. Special mention goes to my collaborator Dr. Silvina Matysiak for performing computational studies to help obtain mechanistic details on my acetylation project.

A heartfelt thank you to all past and present Legleiter lab members, we have always had a fantastic, encouraging and supportive group. Special thanks goes to Dr. Kathleen .A. Burke who initially trained me on how to purify huntingtin protein and to run PDA assays. I would also like to thank Dr. Elizabeth Yates for patiently teaching me most aspects of AFM when I joined the lab. Special thanks go to Dr. Nicole ShamtikoKlingensmith for being a special friend and more like a sister, and for teaching me other 
important aspects of AFM simulations, and Photoshop. Additional thanks to one of the undergraduate student, Pranav Jain. I also thank Dr. James Arndt for helping me with mass spectrometry analysis on one of my projects. Lastly, I would like to thank other lab members (Xiang Gao, Pilkington IV, Sharon Groover, Adewale Adegbuyiro, Faezeh Sedighi for your love and support

Finally, I thankful for such a loving and caring wife, Faith Ruzvidzo Chaibva, who is always able to put a smile on my face. She always believed in me, even when I didn't believe in myself. I would not have made it through the graduate school without her unwavering and unconditional love, and support. I am also thanking my parents (Ishmael and Junior Chaibva) who raised me as a hard worker and sent me to school with their hard earned money. My siblings (Robina, Xavier (late), Aaron, Darlington and Luxton) also played an important role in my education.

Above all, I would like to thank God almighty who gave me the opportunity to come to WVU to purse a funded Ph.D program and gave me creative ideas in my graduate studies. 


\section{Table of Contents}

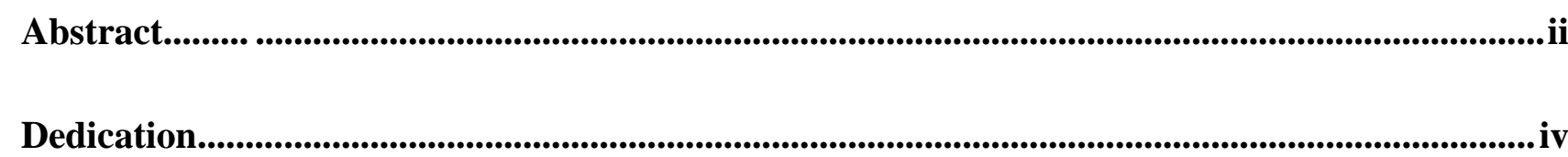

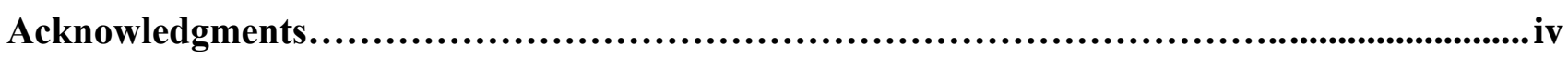

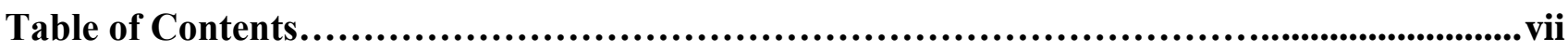

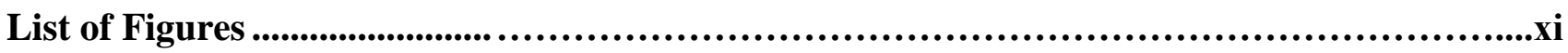

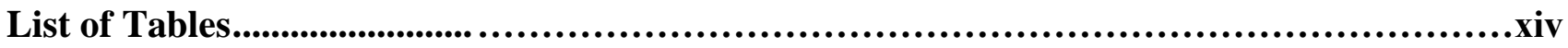

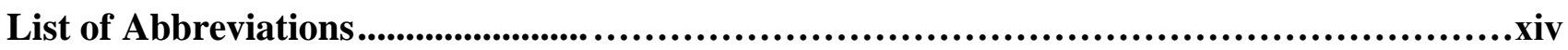

1. Protein aggregation and Huntington's disease ...................................................................................... 1

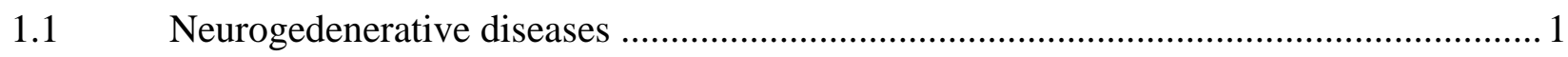

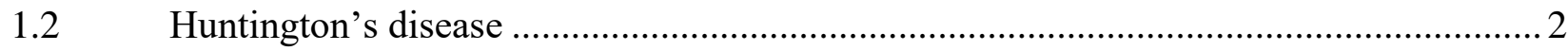

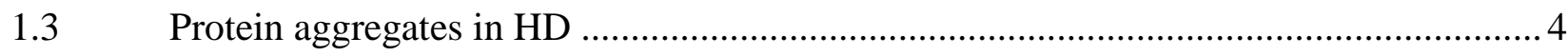

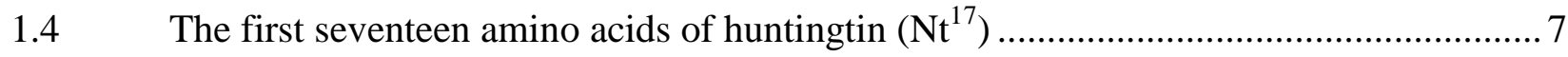

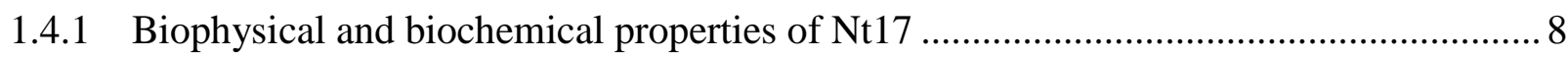

1.4.2 Nt17 as a potential therapeutic target on htt exon 1 ...............................................

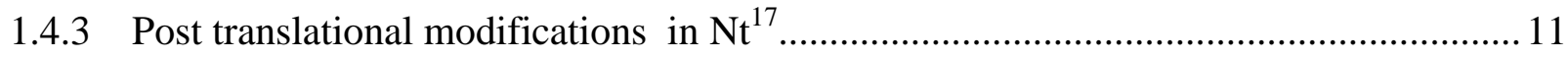

1.4.4 $\mathrm{Nt}^{17}$ mediates huntingtin/lipid interactions with potential implications for toxicity. 14

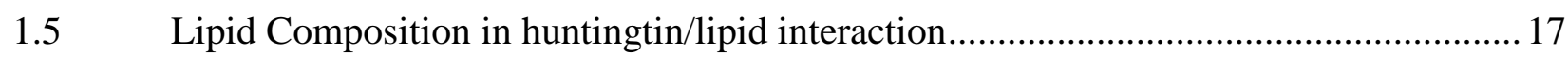

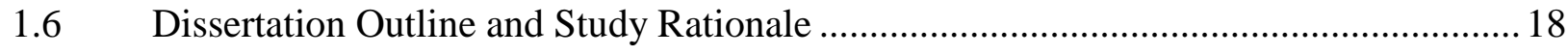


1.7 Atomic Force Microscopy (AFM)

1.8 References

\section{Scanning probe acceleration microscope and the development of supported bilayers}

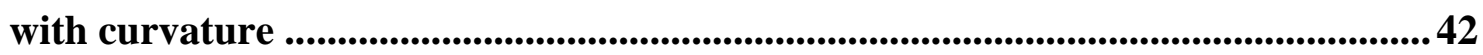

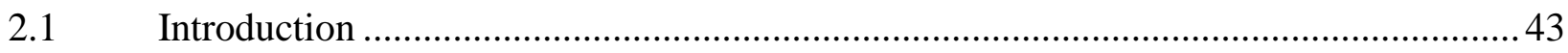

2.2 Numerical model of the entire imaging process of TMAFM in solution.....................46

2.3 Basic principles underlying SPAM …....................................................... 50

2.4 Features of the time-resolved tip/sample force are independent of surface

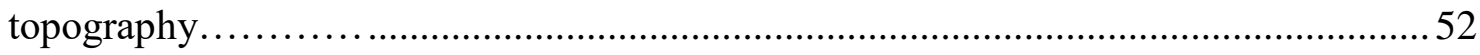

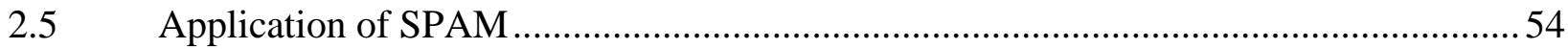

2.6 SPAM in the development of supported lipid bilayers with curvature .......................56

2.6.1 Preparation of total brain lipid extract ........................................................... 56

2.6.2 Preparation of poly-L-lysine coated silicon substrate .........................................5 57

2.6.3 Nanoparticles deposition on silicon substrate ...............................................5

2.6.4 In situ AFM and SPAM imaging conditions ..................................................58

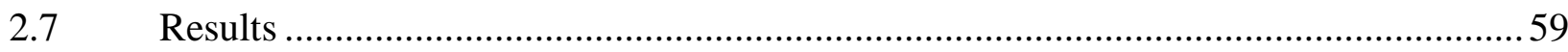

2.7.1 Development of a system of supported flat and curved lipid bilayers .......................59

2.7.2 Validating bilayer formation on beads using imaging forces.................................62

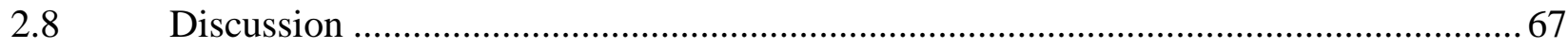

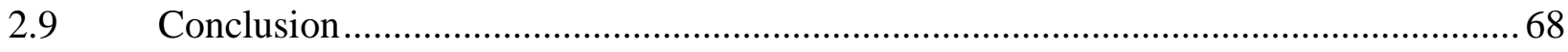


2.10 References

3. Curvature enhances binding aggregation of huntingtin in lipid membranes.............................75

3.1 Introduction:Huntington's disease and importance of membrane curvtaure ..................76

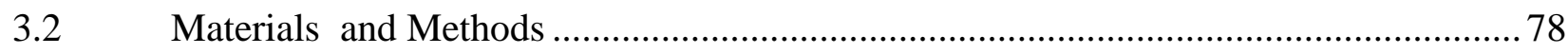

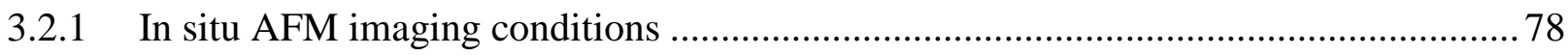

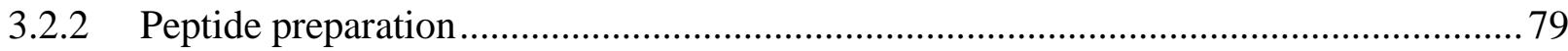

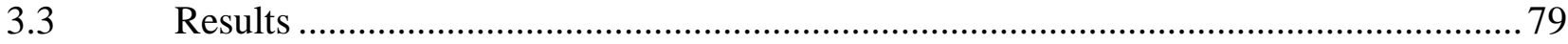

3.3.1 Htt peptides preferentially bind to curved bilayers .................................................... 79

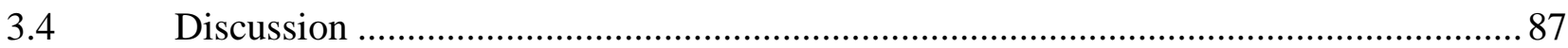

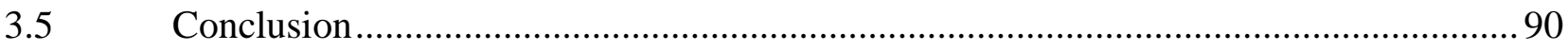

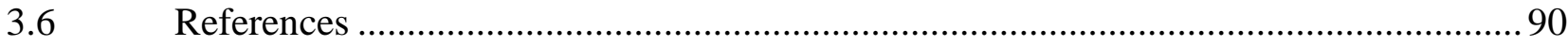

4. Acetylation within the first 17 amino acids of htt exon1 alters aggregation and binding to lipid membranes ...........................................................................................................99

4.1 Introduction: Huntingtin disease and post translational modifications ......................... 100

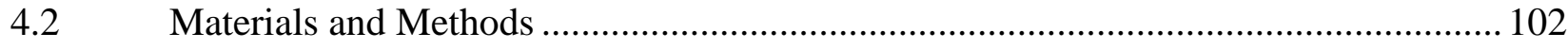

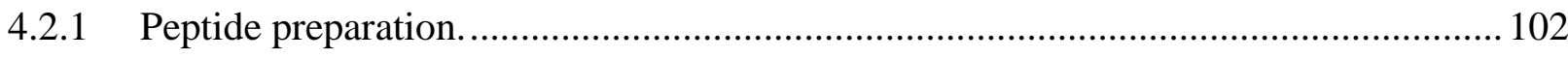

4.2.2 Liquid Chromatography/Tandem Mass Spectrometry ………………........................ 103

4.2.3 Thioflavin T (ThT) assay ……………………………............................................ 104

4.2.4 Purification of GST-htt-exon1 fusion proteins............................................................. 105

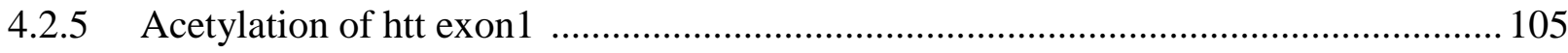




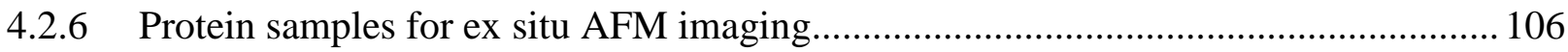

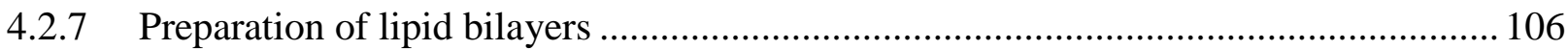

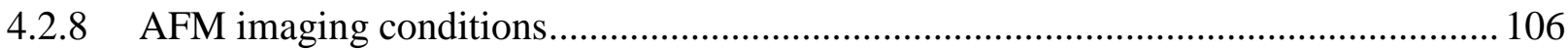

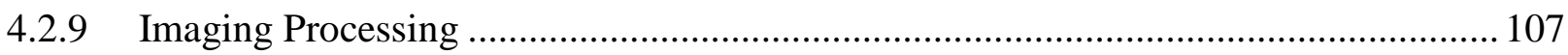

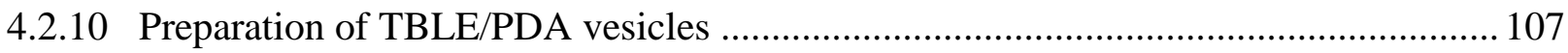

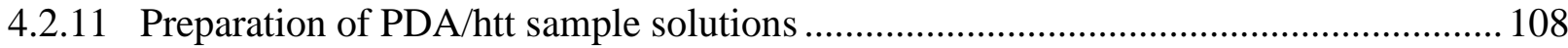

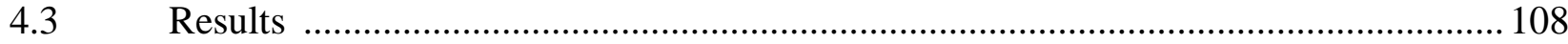

4.3.1 Chemically-induced acetylation occurs at all lysines in $\mathrm{Nt}^{17}$ and inhibits

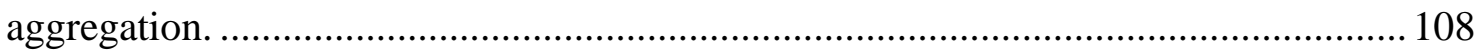

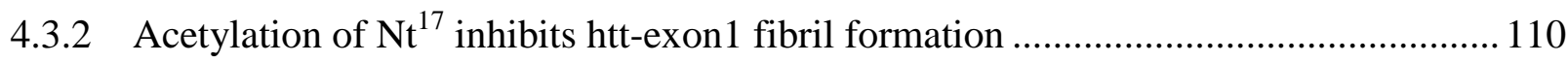

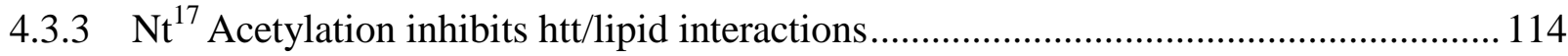

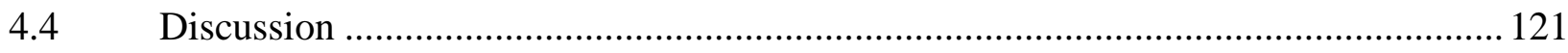

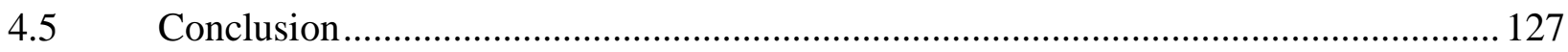

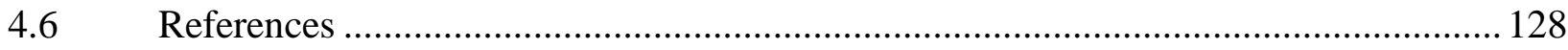

\section{Other relevant post translational modifications and small molecules as potential} modulators of huntingtin aggregation ....................................................................... 144

5.1 Work in progress: GM1 modulates huntingtin aggregation on lipid membranes ........ 145

5.1.1 Cholesterol and GM1 alters the extent of htt-exon1 binding to lipid vesicles as determined by TBLE/PDA binding assay acetylation occurs at all ........................ 146

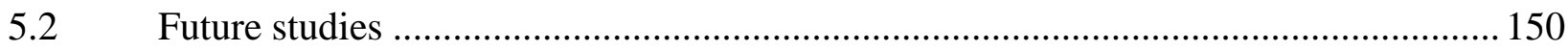


5.2.1 Post translational modifications on huntingtin aggregation

5.2.2 Targeting huntingtin Nt17 with small molecules ......................................................... 151

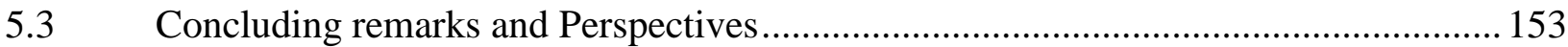

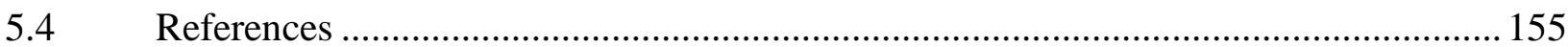




\section{List of figures}

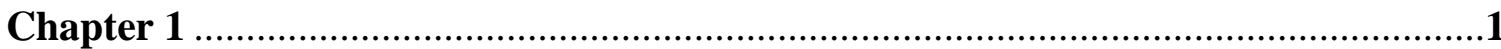

Figure 1. A schematic model for htt aggregation ...............................................

Figure 2. Schematic of important features of of htt exon 1 .......................................4

Figure 3. Basic schematic illustration of an AFM .................................................20

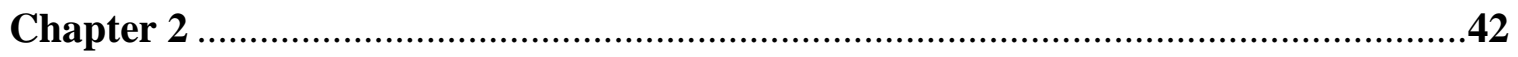

Figure 1. SPAM is a method for reconstructing the time-resolved tip/sample forces associated with TMAFM imaging in solution ....................................................45

Figure 2. Comparison of simulated cantilever position and deflection trajectories in

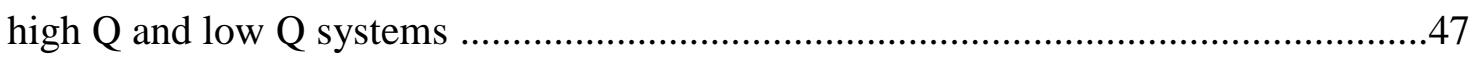

Figure 3. Simulations to determine the effect of surface topography on tip/sample

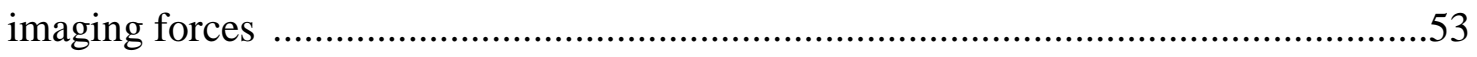

Figure 4. SPAM imaging of a supported lipid bilayer patch on mica ........................55

Figure 5. Time-lapse AFM images taken in solution of a TBLE bilayer forming over silica beads immobilized on silicon

Figure 6. Schematics demonstrating two potential ways the lipid bilayer could be arranged with respect to the beads

Figure 7. Tapping mode AFM simulations demonstrate the relationship between imaging forces and surface mechanical properties

Figure 8. Tapping mode AFM simulations demonstrate the effect of Young's modulus on the measured height of a $50 \mathrm{~nm}$ bead

Figure 9. Maximum forces associated with imaging the surfaces confirm the presence of lipid bilayers covering the beads .65 
Chapter 3

Figure 1. Time lapse AFM images taken in solution of a TBLE bilayer exposed to a synthetic htt exon1 mimicking peptide

Figure 2. AFM image shows that a synthetic htt exon1 mimicking peptide aggregates on and disrupts the flat regions of the lipid bilayer

Figure 3 Three dimensional renderings of model objects with increasingly larger volume are presented

Figure 4. Volume of deposited synthetic htt exon 1-mimicking peptide on the curved or flat regions of the bilayer

Chapter 4

Figure 1. Lysines within $\mathrm{Nt}^{17}$ are accessible for acetylation and impact fibril formation 110

Figure 2. Addition of NHSA to GST-htt-exon1 does not inhibit cleavage of the GST

tag by Factor Xa

Figure 3. Chemical acetylation of $\mathrm{Nt}^{17}$ in htt-exon1 suppresses aggregation

Figure 4. Acetylation of $\mathrm{Nt}^{17}$ in htt-exon1 influences the size of aggregates .114

Figure 5. Acetylation of $\mathrm{Nt}^{17}$ modulates the interaction between htt-exon1 and lipid membranes 115

Figure 6. Exposure to htt-exon1disrupts TBLE bilayers morphologically

Figure 7. Acetylation of $\mathrm{Nt}^{17}$ does not alter the size of oligomers of htt-exon1 on lipid bilayers .120

Figure 8. Acetylation-mimicking mutations reduce the affinity of $\mathrm{Nt}^{17}$ for lipid vesicles .121 
Chapter 5

Figure 1. Percent colorimetric response (\%CR) of TBLE/PDA vesicles containing various amounts of cholesterol upon exposure to htt-exon1

Figure 2. Percent colorimetric response (\%CR) of TBLE/PDA vesicles containing various amounts of GM1 upon exposure to htt-exon1 .......................................148

Figure 3. GM1 alters the interaction of htt with supported lipid bilayers

Figure 4. Representative AFM images of htt exon 1 at different type points after incubation with polpP and $\mathrm{Nt}^{17}$ 


\section{List of tables}

Table 1. Estimation of the \% error associated with tip convolution

\section{List of symbols and abbreviations}

A

$A_{\mathrm{o}}$

$a_{D M T}$

$a_{0}$

AFM

$b$

CD

$\% \mathrm{CR}$

$D_{0}$

DLVO

DMT

E

E. coli

$F_{\text {ext }}$

$F_{\max }$

$F_{\text {total }}$

GST

$H$

HCD

HD cantilever tapping amplitude

cantilever free tapping amplitude,

interatomic distance

drive oscillation amplitude

atomic force microscopy

damping coefficient

circular dichroism

percent colorimetric response

resting position of the cantilever base

Derjaguin-Landau-Verway-Overbeek

Derjaguin-Muller-Toporov

Young's modulus

Escherichia coli

tip/sample tapping force

maximum tapping force

total tapping force

glutathione S-transferase

Hamaker constant

Higher-energy collisional dissociation

Huntington's disease 


\begin{tabular}{|c|c|}
\hline HFIP & hexafluoroisopropanol \\
\hline $\mathrm{Htt}$ & huntingtin \\
\hline htt exon1(51Q) & huntingtin exon1 with 51 glutamines \\
\hline IPTG: & Isopropyl $\beta$-D-1-thiogalactopyranoside \\
\hline$k$ & spring constant \\
\hline $\mathrm{kDa}$ & KiloDalton \\
\hline$\kappa_{\text {eff }}$ & effective indentation constant \\
\hline KK-Q35-P10-KK & $\begin{array}{l}35 \text { glutamines followed by } 10 \text { prolines flanked by } 2 \text { lysines } \\
\text { on each side }\end{array}$ \\
\hline K6Q & lysine mutated to glutamine \\
\hline$m_{\text {eff }}$ & mass of the cantilever \\
\hline MS & mass spectrometry \\
\hline NHSA & sulfo-N-hydroxysuccinimide \\
\hline $\mathrm{Nt}^{17}$ & the first $17 \mathrm{~N}$-terminal amino acids of the huntingtin \\
\hline PBS & phosphate-buffered saline \\
\hline polyP & polyproline \\
\hline polyQ & polyglutamine \\
\hline PTMs & post translational modifications \\
\hline PDA & polydiacetylene \\
\hline$Q$ & quality factor of the cantilever \\
\hline$R_{t i p}$ & radius of the cantilever tip \\
\hline RMS & root mean square \\
\hline$S$ & set-point ratio \\
\hline
\end{tabular}




$\begin{array}{ll}\text { SPAM } & \text { scanning probe acceleration microscopy } \\ \text { TBLE } & \text { total brain lipid extract } \\ \text { TFA } & \text { trifluoroacetic acid } \\ \text { ThT } & \text { thioflavin T } \\ \text { TMAFM } & \text { tapping mode atomic force microscopy } \\ \text { Tris } & \text { Tris(hydroxymethyl)aminomethane } \\ \Omega & \text { drive frequency } \\ \omega_{o} & \text { resonance frequency } \\ \omega_{\text {oper }} & \text { operating frequency } \\ x & \text { cantilever deflection } \\ y & \text { cantilever deflection } \\ z & \text { position of the cantilever with respect to the surface }\end{array}$




\section{Protein aggregation and Huntington's disease ${ }^{1}$}

\subsection{Neurodegenerative diseases}

The molecular pathway associated with a vast number of neurodegenerative diseases, including Huntington's disease (HD), Alzheimer's disease (AD) and Parkinson's disease (PD), is characterized by protein misfolding, resulting in the aggregation of the misfolded protein, and its deposition, and accumulation in cellular compartments as nanoscale aggregates $[1,2]$. Proteins are synthesized as linear chains of several amino acids, and most properties adopt a well-defined three dimensional compact structure during folding for them to be functional. The main driving force for protein folding is the tendency for hydrophobic residues within the protein surface to interact together rather than with water, a phenomenon known as the hydrophobic effect. This leads to the burial of nonpolar side chains in the interior of proteins, leading to the "collapse" of the protein from an extended coil to a more defined, globular structure. Proteins are at risk of misfolding in which a protein is trapped in a local energy minimum and become misfolded. These misfolded proteins can follow a complex pathway that leads to the formation of fibrils rich in $\beta$-sheet structures via intermediate species such as oligomers (Fig. 1), and these aggregates will eventually accumulate as inclusions in the brain of diseased victims. The deposition of these inclusion bodies is the hallmark of many neurodegenerative diseases. It remains controversial whether the small, potentially diffusible assemblies, or mature amyloid fibrils, are primary culprits in neuronal dysfunction and death [3-5].

\footnotetext{
${ }^{1}$ This chapter includes the review that was co-authored with James Arndt with equal contribution: The Emerging Role of the First 17 Amino Acids of Huntingtin in Huntington's disease.

1. Introduction
} 


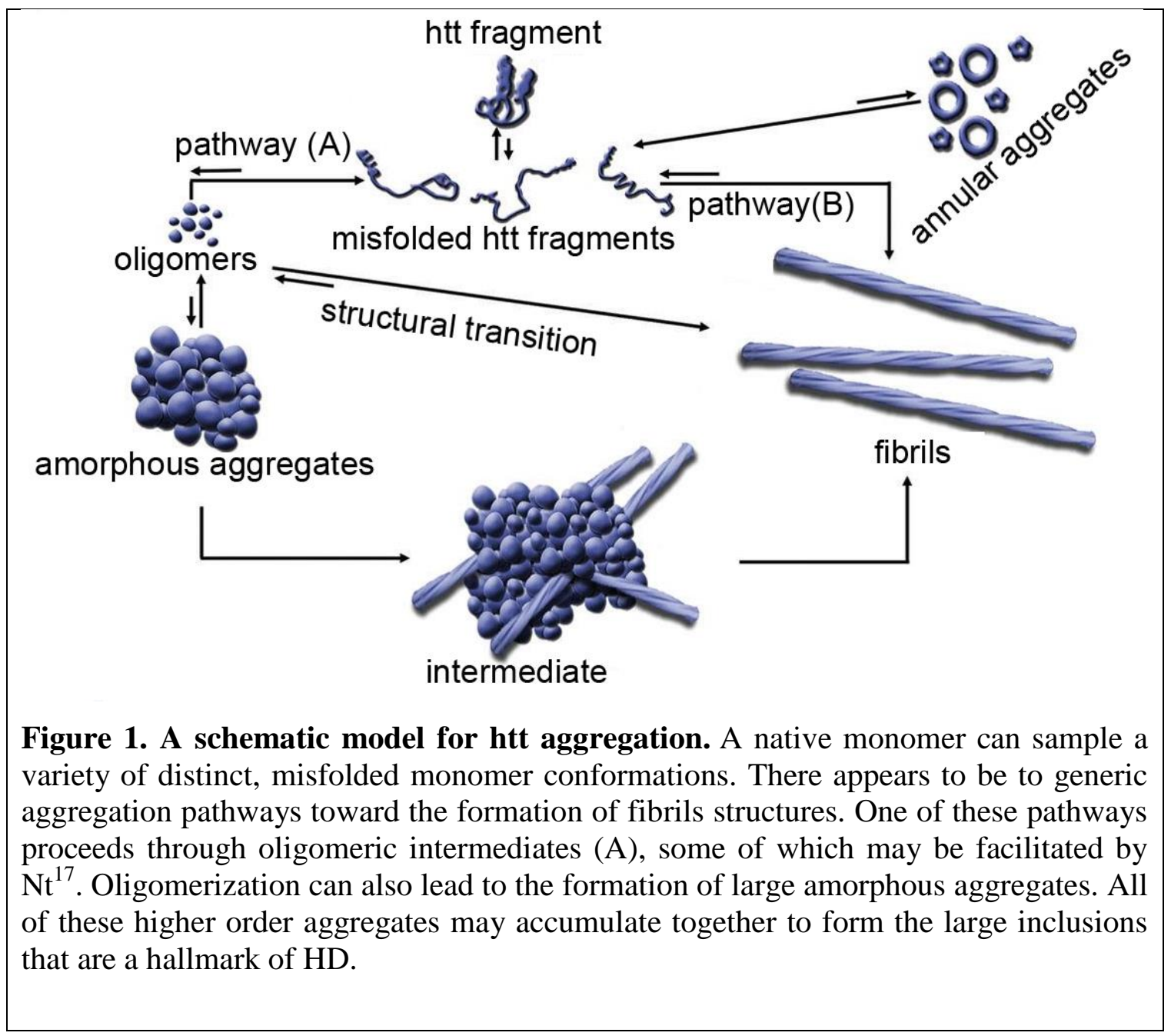

In this introductory chapter, the Huntington's disease protein will be discussed, including the role of the protein's first seventeen amino acids on modulating its aggregation, kinetically and morphologically on lipid membranes.

\subsection{Huntington's disease (HD)}

HD is a fatal, progressive neurodegenerative disorder with well-established origins and affects specific neurons in the brain. This monogenic disorder is caused by a single mutation in the IT15 gene that code for Huntington protein, huntingtin (htt). The

\section{Introduction}


mutation occurs as a CAG trinucleotide expansion in the gene. CAG codes for the amino acid glutamine, and therefore, the mutation leads to an expansion of a polyglutamine (polyQ) tract near the $\mathrm{N}$-terminus of the protein htt [6]. This polyQ expansion directly leads to htt aggregation into fibrils and a variety of other aggregates [7-9], as shown in Fig. 1. While the exact mass of htt is dependent on the size of the polyQ domain, fulllength htt consists of 3144 amino acids (figure full length), 67 exons, and is approximately $350 \mathrm{kDa}$ in weight [6]. The polyQ domain begins at the $18^{\text {th }}$ residue of htt and is contained in exon 1. For the studies presented here, the htt fragment (htt exon1) not the full protein was used .Several lines of evidence suggested that $\mathrm{N}$-terminal fragments comparable to exon 1 are directly involved in HD [10-15]. For instance, expression of htt exon1 with an expanded polyQ tract causes a progressive neurological phenotype (including incoordination, gait instability, cognitive abnormality, weight loss, progressing to early death) in R6/2 transgenic mice $[13,16]$. More recent studies demonstrated that, N-terminal fragments comparable to exon1 are detected in knock-in mouse models expressing full length htt [14], and fragments of the size of exon1 have been detected in HD patients [17]. Finally, it has not been possible to recombinantly express and purify the full htt protein. The polyQ domain is flanked by the first 17 amino acids at the $\mathrm{N}$ terminus $\left(\mathrm{Nt}^{17}\right)$ of the protein and by a polyproline (polyP) stretch on its $\mathrm{C}$-terminal side (Fig.2a). One of the key aspects of HD is that there is a threshold length of the polyQ domain required for disease with a tight correlation between both the age of onset and severity of disease with the increasing length of the polyQ expansion. Specifically, individuals with CAG repeat lengths less than 35 in their DNA sequence will not get the

\section{Introduction}




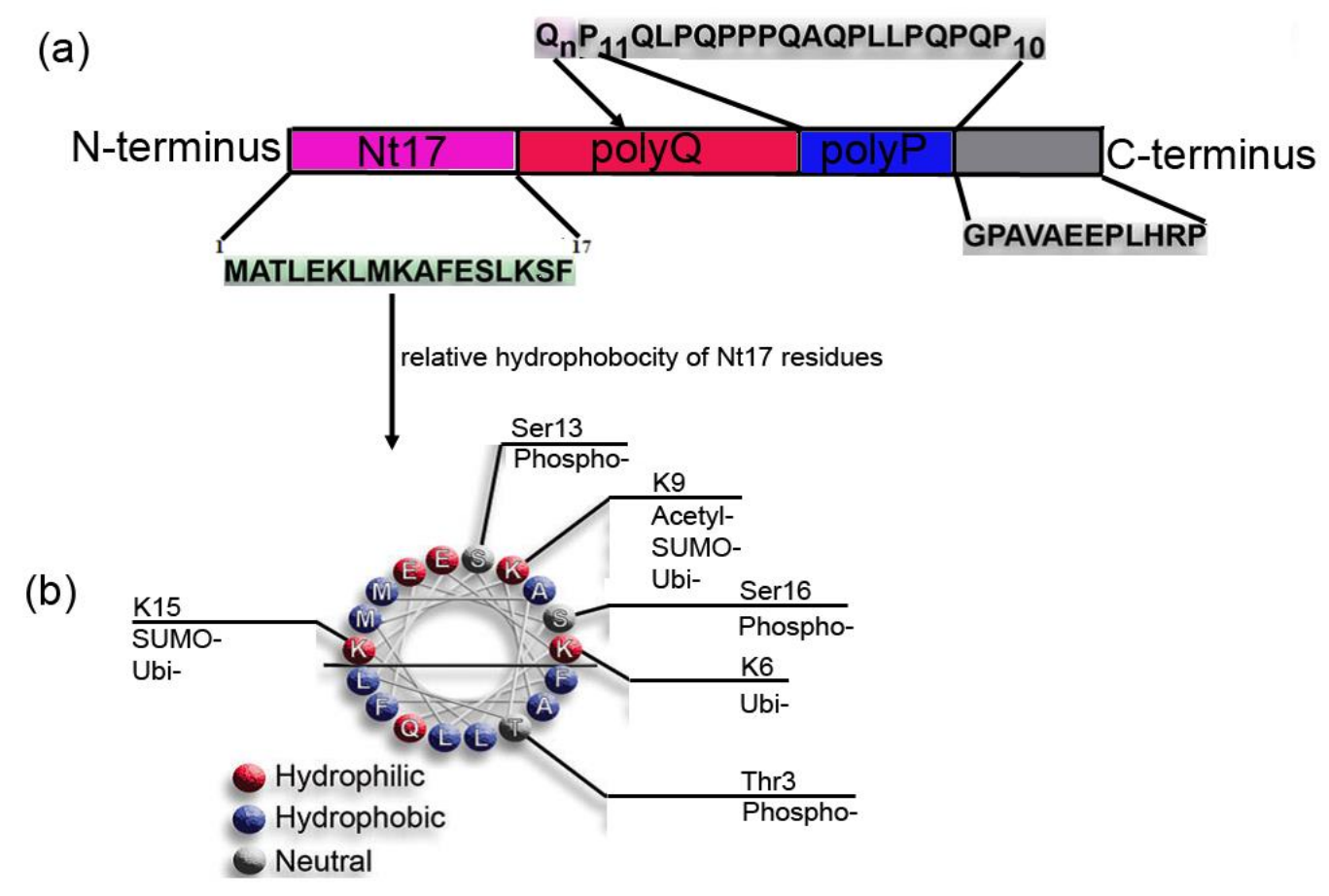

Figure 2. Schematic of important features of of htt exon1, with (a), showing $\mathrm{Nt}^{17}$ (purple), polyQ (red) and the polyP (blue) regions. b) View down the barrel of the $\alpha$ helix showing relative hydrophobicity of each residue as well as the sites of reported posttranslational modifications. The colors on (a) don't correspond to (b)

disease, 35-39 repeats may elicit disease, 40-60 repeats cause adult onset, and juvenile forms of HD are associated with repeats greater than 60 [18-20]. Symptoms typically begin between 35-50 years, followed by 15-20 years of progression before death [21].

\subsection{Protein aggregates in HD}

The role of aggregates in HD is a complex issue and remains to be clearly understood. Biochemically and biophysically, it has been demonstrated that htt fragments containing expanded polyQ tracts readily form detergent-insoluble protein aggregates with characteristics of amyloid fibrils $[22,23]$. Furthermore, the rate of aggregation into

\section{Introduction}


fibrils is highly correlated with polyQ length in synthetic peptides and htt exon1 fragments [24]. This correlation between aggregation rate and polyQ length has been recapitulated in cell culture models expressing htt fragments [25-27]. While it is clear that htt with expanded polyQ tracts assemble into fibrils, htt also forms spherical and annular oligomeric structures [9, 28, 29]. It is therefore evident that htt can form a variety of aggregate structures; however, the precise mechanisms by which these structures induce toxicity and neurodegeneration is unclear. Precise characterization of the possible aggregation pathways and the resulting heterogeneous mixtures of aggregates, as well as factors that modulate protein aggregation is a vital step in understanding htt aggregation.

Extensive research has been performed to determine the kinetics of htt aggregation and to determine specific aggregate species on and off pathway to fibril formation [24]. In this regard, several aggregation pathways have been proposed for the formation of fibrils of polyQ-containing proteins. Two of the more prominent aggregation schemes are: 1) re-arrangement of a monomer to a thermodynamically unfavorable conformation that directly nucleates fibril formation and 2) the formation of soluble oligomeric intermediates that slowly undergo structural re-arrangement into a $\beta$ sheet rich structure leading to fibrils (Fig. 1). The precise nature of the oligomeric aggregates associated with the second scheme can be quite heterogeneous [30,31], and there could be a variety of oligomeric species that are off-pathway to fibril formation. While polyQ peptide fibrils share many classical features associated with amyloids, initial reports using pure polyQ peptides supported the nucleation-elongation model for the formation of polyQ fibrils $[32,33]$. Subsequent studies have suggested other potential mechanisms associated with the potential of smaller aggregates of polyQ proteins 1. Introduction 
appearing prior to nucleation of fibril formation [34, 35]. Small oligomers, displaying various degrees of stability, of polyQ peptides with various glutamine lengths were observed by atomic force microscopy (AFM) [7]. Studies of polyQ peptides that were interrupted by other amino acids further support the formation of oligomers [36]. While many oligomers were observed to directly initiate fibril formation when individually tracked in solution, many fibrils appeared to form without any obvious oligomeric precursors [7]. Such observations suggest that these aggregation pathways are not mutually exclusive, making the situation considerably more complicated. AFM studies of a variety of htt-exon 1 fragments have shown that aggregation reactions result in heterogeneous mixtures of distinct aggregate morphologies in a polyQ length- and concentration-dependent manner [7]. Novel oligomers of htt exon1 have also been identified in an in vitro mammalian system [30]. Due to the complexity of htt aggregation, there is no clear consensus on the aggregate form(s) that underlie toxicity, and there likely exist bioactive oligomeric aggregates undetectable by traditional biochemical and electron microscopic approaches that precede symptoms. While identification of the toxic specie(s) of htt that trigger neurodegeneration in HD remains elusive, such species might exist in a diffuse, mobile fraction rather than in inclusion bodies [37].

Mutant htt is detected in patients' brains predominantly as microscopic inclusion bodies in the cytoplasm and nucleus [13]; however, htt is also associated with a variety of membranous organelles, including mitochondria, endoplasmic reticulum, tubulovesicles, endosomes, lysosomes and synaptic vesicles [16, 38-41]. The precise mechanisms by which htt aggregates are toxic to neural cells, leading to the extensive cellular destruction 1. Introduction 
that is the hallmark of $\mathrm{HD}$, remain unclear. As a result, there is a pressing need to understand factors that modulate htt aggregation, contribute to pathogenesis, and could potentially serve as therapeutic targets. The $\mathrm{Nt}^{17}$ could be very important in modulating htt aggregation.

\subsection{The first seventeen amino acids of huntingtin $\left(\mathrm{Nt}^{17}\right)$}

A potential mechanism by which $\mathrm{Nt}^{17}$ influences toxic htt aggregation is that the formation of abnormal aggregates of mutant htt is directly influenced by this domain. Indeed, $\mathrm{Nt}^{17}$ has been implicated in driving the initial phases of htt exon 1 interaction [39, 42-45]. Several mechanisms have been proposed that are mediated by $\mathrm{Nt}^{17}$. Most agree that the initial phase of aggregation when $\mathrm{Nt}^{17}$ is present begins with self-association of this domain, resulting in the formation of small, $\alpha$-helix rich oligomers [46-48]. As such, it appears that $\mathrm{Nt}^{17}$ promotes fibril formation via the oligomer mediated pathway. In a cellular environment, pathogenic htt exon 1 oligomer populations do not appreciably change even with recruitment of monomers into inclusion bodies, suggesting that the formation of oligomeric species mediated by $\mathrm{Nt}^{17}$ is the rate-determining step $[49,50]$. Since oligomers are widely considered to be the toxic species in Huntington's disease, the promotion of oligomerization by $\mathrm{Nt}^{17}$ could have potentially important consequences of htt-related toxicity. PolyQ peptides that do not have the N-terminal flanking sequence tend not to form oligomeric intermediates and as a result, proceed directly to fibrillar aggregates [51, 52]; although, some pure polyQ-peptide oligomers have been observed to form when aggregating on a surface $[7,53]$. The addition of a myc-tag preceding $\mathrm{Nt}^{17}$ in full htt exon 1 reduces the formation of oligomers in vitro without changing the rate of

\section{Introduction}


fibril formation when compared to exon 1 proteins of similar polyQ length that lacked this myc-tag [54], suggesting that the ability of $\mathrm{Nt}^{17}$ to promote oligomerization can be interfered with by steric hindrances. Such findings seem to suggest that $\mathrm{Nt}^{17}$ could a good target for therapeutic intervention.

\subsubsection{Biophysical and biochemical properties of $\mathrm{Nt}^{17}$}

The $\mathrm{Nt}^{17}$ domain can form an amphipathic $\alpha$-helix (AH) that is conserved in at least some aggregate forms of htt $[44,52,55]$. There are several important biophysical properties associated with AHs [56], but in particular, AHs are often associated with binding of lipid membranes $[57,58]$. Briefly, an AH consists of a predominately hydrophilic face and a predominately hydrophobic face (Fig. 2b). AHs have been shown to have several functional properties such as preferentially sensing and binding highly curved membrane by detecting defects induced by curvature [59]. Due to their ability to weakly bind membranes, their interaction with membranes is easily regulated. $\mathrm{Nt}^{17}$ has been shown to sequester truncated htt exon1 peptides to regions of curvature on supported lipid bilayers [60]. The structure of an $\mathrm{AH}$ is compatible with the formation of a variety of helix bundles in aqueous environment that is driven by maximizing the interaction of the hydrophobic faces of adjacent helices [61, 62]. Due to this, the association of $\mathrm{Nt}^{17}$ via the formation of interacting $\mathrm{AHs}$ has been proposed to play a role in the initial stages of $\mathrm{htt}$ aggregation. The $\mathrm{Nt}^{17}$ primary sequence contains three positively charged lysine residues at positions 6,9 , and 15 . Residues 6 and 15 lie at the boundary between the hydrophilic and hydrophobic faces in a theoretical AH that extends the entire length of $\mathrm{Nt}^{17}$ (Fig. 2b). Additionally, two methionine, two serine, and two

\section{Introduction}


glutamic acid residues also reside on the hydrophilic face. The hydrophobic face consists of two phenylalanine residues, three leucine residues, and an alanine residue, with a leucine and a phenylalanine at each side of the boundary. It would appear that residues in the boundary region are critical in lipid membrane binding and intermolecular interaction. Simulation of lipid membrane association show K6 and K15 are strong hydrogen bond donors with lipid bilayers [63]. Similarly, K6 was found to be protected from an aggregated state by solution phase deuterium exchange mass spectrometry [64]. These studies point to critical interactions involving amphipathic helices that can potentially be involved in the formation of the previously mentioned oligomeric precursors to fibril formation that are mediated by $\mathrm{Nt}^{17}$. Compelling evidence demonstrates that the $\alpha$-helix is conserved in fibrils $[44,64,65]$. Additionally, ssNMR chemical shifts indicate residues $4-11$ are in an $\alpha$-helical arrangement within a fibril structure, while residues $17-19$ have a clear $\beta$-sheet structure [44]. While there is little doubt that $\mathrm{Nt}^{17}$ is capable of forming an $\mathrm{AH}$, under what conditions $\mathrm{Nt}^{17}$ adopts this structure in monomeric htt and the related consequences for aggregation are of critical importance.

\subsection{2 $\mathrm{Nt}^{17}$ as a potential therapeutic target on htt exon 1}

From the ongoing discussion, it is clear that $\mathrm{Nt}^{17}$ has been implicated in the formation of the toxic intermediates, it represents a novel target to inhibit (or alter) aggregate formation, thereby possibly alleviating htt-mediated toxicity. A more practical means of inhibiting $\mathrm{N}$-terminal aggregation is through structural complexation, either by small molecules, molecular chaperones or an antibody-antigen that interacts directly with this domain [66-71]. Interestingly, free $\mathrm{Nt}^{17}$ (lacking any polyQ) has been used to inhibit

\section{Introduction}


$\mathrm{Nt}^{17}$-mediated formation of $\mathrm{Nt}^{17} \mathrm{Q}_{37} \mathrm{P}_{10} \mathrm{~K}_{2}$ peptides in vitro [51, 52]. The mechanism by which $\mathrm{Nt}^{17}$ inhibits amyloid formation appears to be identical to the mechanism by which nucleation occurs, with the caveat that $\mathrm{Nt}^{17}$ alone does not contain an amyloidogenic polyQ tract. This increases the distance between adjacent polyQ tracts and the energy for polyQ fibrillization to occur [52]. It has also been known for some time that molecular chaperones can modulate htt aggregation [72,73]. Many of these molecular chaperones, including Hsc79 [74] and Tric [68, 69], have been shown to bind directly to $\mathrm{Nt}^{17}$. Specifically ,it was found out that an intrinsically disordered chaperone, HYPK interacts with $\mathrm{Nt}^{17}$ in htt and modulates aggregate formation and cytotoxicity in neuronal cell lines [75].

Another potential therapeutic strategy that has been extensively studied is the use of antibodies; unlike peptide therapeutics and inhibitors, antibodies are specific only for the target antigen. Several antibodies exist for htt exon 1, each recognizing distinct domains and potentially distinct monomers or aggregates [31, 76-79]. In the past decade, intracellular antibodies, or intrabodies, for $\mathrm{Nt}^{17}$ have been developed that inhibit htt exon 1 fibrillization and cytotoxicity in vitro and cellular huntingtin model, two of which are scFv and $\mathrm{V}_{\mathrm{L}}$ 12.3.[66, 67, 80]. When co-transfected with htt exon 1 (104Q) into COS-7, BHK-21, and HEK-293 cells, $\mathrm{C} 4 \mathrm{scFv}$ was shown to decrease aggregate formation relative to control groups that were not transfected with the intrabody [80]. $\mathrm{C} 4 \mathrm{scFv}$ bound to htt exon 1 structures of non-pathogenic length (25Q), as well, which indicates the polyQ conformation does not play a role in intrabody binding, and that the most likely binding target is $\mathrm{Nt}^{17}$ [80]. These studies show that structural inhibition of $\mathrm{Nt}^{17}$ is certainly a viable means of inhibiting aggregation and, potentially, htt exon 1 toxicity. 1. Introduction 


\subsubsection{Post translational modifications (PTMs) in $\mathrm{Nt}^{17}$}

$\mathrm{Nt}^{17}$ also contains numerous sites that can be post-translationally modified, with some sites associated with multiple potential modifications (Fig. 2b) [81-83]. These PTMs appear to have a profound effect on htt function and translocation [84-86], as well as toxicity associated with htt containing expanded polyQ domains [81, 82, 87-89]. Most prevalent forms of PTMs occurring in $\mathrm{Nt}^{17}$ are phosphorylation [43, 89-92], acetylation [93], ubiquitination [94], and SUMOylation [81, 87]. Other htt modifications such as palmitoylation [95] and transglutamination [96] have also been observed.

The htt $\mathrm{Nt}^{17}$ domain has three potential phosphorylatable sites: threonine 3, (Thr3), serine 13 (Ser13), and serine (Ser16) (full length htt can also be phosphorylated at serine residues downstream from the $\mathrm{N}$ terminal domain. Numerous studies have reported that htt phosphorylation is associated with reduced levels of mutant htt toxicity [83, 89, 91, 92, 97-99]. Mutating $\mathrm{Thr} 3$ to aspartate to mimic phosphorylation enhances the propensity of htt to aggregate in cultured cells and resulted in reduced lethality and neurodegeneration in Drosophila models of HD [89]. Htt containing phospho-serine residues at 13 and 16 aggregate in a similar fashion to serine to aspartate phosphomimetic mutations, demonstrating that serine phosphomimetic mutations are valid mimics for studying the impact of htt phosphorylation on aggregation. Using a variety of combinations of serine phosphorylation mimics, it was established that serine to aspartate mutations significantly slows the formation htt aggregates and reduces the stability of the

fibril structure $[43,92]$. When Ser13 and Ser16 were mutated to aspartate in the $\mathrm{Nt}^{17}$ domain of transgenic mice that expresses full length htt with $97 \mathrm{Q}$, visible htt inclusions

\section{Introduction}


within the brain were reduced, and HD-like behavioral phenotypes were reduced at 12 months of age [92]. Altering the phosphorylation state of htt (specifically at Ser13) indirectly can also impact expanded htt related toxicity, as casein kinase 2 inhibitors reduced Ser13 phosphorylation with a subsequent enhancement of htt-related toxicity in high content live cell screenings [100]. Furthermore, ganglioside, GM1, triggers htt phosphorylation at Ser13 and Ser16, diminishing htt toxicity while restoring normal motor behavior in transgenic mice [90]. Collectively, this finding further support the notion that $\mathrm{Nt}^{17}$ plays an important role in htt aggregation and, more specifically, that altering the phosphorylation state of $\mathrm{Nt}^{17}$ may be a viable therapeutic strategy.

Htt can also undergo SUMOylation, ubiquitination, and acetylation on specific lysine residues within $\mathrm{Nt}^{17}$. These potential sites include lysine residues at position 6,9 , and 15. SUMOylation, specifically, has been implicated in both cell and animal models to be involved in HD pathogenesis [81, 87]. SUMOylation at lysine 6 (K6) and lysine 9 (K9) stabilizes htt exon1 fragments, reducing aggregation in cultured cells and exacerbating toxicity in Drosophila HD models [81]. Rhes, a small G protein, preferentially binds mutant htt over wild type and acts as a SUMO E3 ligase [101]. The subsequent SUMOylation by Rhes increases soluble levels of htt and enhances neurotoxicity [101]. Ubiquitination is known to compete for the same lysine residues as SUMOylation, but functionally, it is associated with tagging proteins for degradation by the ubiquitin proteasome system (UPS), thereby reducing the toxicity of the mutant htt [102]. However, the presence of ubiquitin in neuronal intranuclear inclusions in the HD striatum and cortex shows that the detoxification mechanisms by ubiquitination is incomplete for most proteins [16]. Proteomic mapping by MS verified within $\mathrm{Nt}^{17}$ that 1. Introduction 
K9 is appreciably acetylated in mammalian cell lysates [103]. While the role of K9 acetylation has not yet been fully evaluated, acetylation of lysine 444 (K444) has been implicated in autophagic removal of mutant htt for clearance leading to neuroprotection in C.elegans HD model [93]. Perhaps, acetylation within $\mathrm{Nt}^{17}$ plays a similar role, but the impact of acetylation within $\mathrm{Nt}^{17}$ on aggregation and toxicity of htt has not been fully elucidated.

Finally, PTMs can occur in tandem, directly affecting other PTMs. For instance, htt SUMOylation can be modulated by phosphorylation, explaining why Ser13 and Ser16 phosphomimics in htt were found to modulate SUMO-1 modification both in cells and in vivo $[91,92]$. SUMOylation is a transient process, and $\mathrm{Nt}^{17}$ lysines are predicted as low probability SUMO sites. Therefore, one way to enhance Nt17 SUMO-1 modification is to use serine phosphomimetic mutants [87]. Interestingly, these phosphomimics also promote K6 htt SUMOylation, which may represent one way to regulate K9 acetylation [87]. SUMOylation and ubiquitination can also influence each other by competing for the same $\mathrm{Nt}^{17}$ lysine 6,9 and 15 [81], implying that one modification could be used to control or enhance the other. Understanding how $\mathrm{Nt}^{17}$ PTMs impact htt and modulate each other could lead to therapeutic intervention with the potential implications to HD. Here, the acetylation of htt and its role in modulating htt aggregation will be presented.

\subsection{4 $\mathrm{Nt}^{17}$ mediates htt/lipid interactions with potential implications for toxicity.}

Another potential role of $\mathrm{Nt}^{17}$ in htt-related toxicity is that $\mathrm{Nt}^{17}$ directly dictates whether, and to what extent, toxic protein aggregates interact with cellular and 1. Introduction 
subcellular compartments, such as organelles. These interactions would be mediated by $\mathrm{Nt}^{17}$ 's ability to bind membranes comprised predominately of lipids and could play an important role in htt trafficking. These membranes may also be direct targets of htt aggregates, that is, htt aggregates may alter membrane structure and stability as part of a molecular mechanism of toxicity. A considerable number of observations directly and indirectly support such notions. Htt has been implicated in the transport of lipid vesicles (endocytic, synaptic or lysosomal), especially along microtubules [104-107]. Approximately $50 \%$ of endogenous htt were found to partitions with membranes after subcellular fractionation of neuron-like clonal striatal cells [108]. In vitro experiments have shown that brain lipids large unilamellar vesicles (LUV) interacted with htt exon1 fragments and enhanced fibril formation of htt aggregates [109], highlighting the importance of $\mathrm{Nt}^{17}$ in lipid/protein binding. Expanded polyQ-conferred nuclear localization of htt appears to require additional htt sequences such as $\mathrm{Nt}^{17}$ [110], and $\mathrm{Nt}^{17}$ itself has been implicated to function as a nuclear export signal [111]. Htt also associates with acidic phospholipids [112] which could play a role in nucleating aggregation. Furthermore, htt localizes to brain membrane fractions [113] which are primarily comprised of lipids.

There are a variety of membrane-associated functions attributable to htt in which the lipid-binding properties of $\mathrm{Nt}^{17}$ may play a role. These functions include cellular adhesion [114], motility [115, 116], cholesterol and energy homeostasis [117], and as a molecular scaffold for coordination of membrane and cytoskeletal communication [112] as well as facilitating microtubule-dependent vesicle transport [118]. $\mathrm{Nt}^{17}$ facilitates the trafficking of htt exon 1 to membranes associated with the ER, autophagic vacuoles, 1. Introduction 
mitochondria, and Golgi [39, 119]. Membrane curvature sensing, facilitated by AH structural characteristics of $\mathrm{Nt}^{17}$, may play a mechanistic role in these functions [60]. Additional evidence that $\mathrm{Nt}^{17}$ directly interacts with lipid membranes was provided by the observation that the binding of htt exon 1 to large unilamellar vesicles composed of phosphatidylcholines (PC) or a mixture of PC and phosphatidylserines (PS) enhanced the helical content of $\mathrm{Nt}^{17}$ [39]. These changes in helical content were blocked by changing the eighth residue of $\mathrm{Nt}^{17}$ to a proline [39]. Through solid state nuclear magnetic resonance structural analysis studies, $\mathrm{Nt}^{17}$ of htt has been found to have an amphipathic alpha helical structure with membrane binding properties [120,121], where it transition structurally from random-coil in solution to an $\alpha$-helical structure upon binding lipid bilayers. $\mathrm{Nt}^{17}$ in synthetic polyQ peptides was required for synthetic polyQ peptides to bind model brain lipid extract membranes [53]. Synthetic polyQ peptides with a variety of flanking sequences readily form fibrils in solution or solid surfaces such as mica, but lipid membranes stabilize discrete oligomeric aggregates of polyQ peptides that contain $\mathrm{Nt}^{17}$ [53]. However, both $\mathrm{Nt}^{17}$ and a C-terminal polyP domain were required for polyQ peptides to destabilize membrane structure, leading to leakage. Computational studies further support the important role of $\mathrm{Nt}^{17}$ in $\mathrm{htt} /$ lipid interactions and also provide evidence that the presence of both $\mathrm{Nt}^{17}$ and polyP enhance the interaction of htt with bilayers [63]. The cooperative effect of $\mathrm{Nt}^{17}$ and the polyP domain in disrupting membranes may be particularly important in light of observed interactions between these two domains in a cellular environment that is altered with increasing polyQ length [122]. The binding and disruption of lipid membranes by full htt exon 1 constructs can be

\section{Introduction}


partially inhibited by the addition of $\mathrm{N}$-terminal tags, further supporting a prominent role of $\mathrm{Nt}^{17}$ in these phenomena [123].

In terms of a potential role in htt-related toxic mechanisms, the accumulation of monomeric htt and/or prefibrillar aggregates directly binding lipid membranes has been shown to disrupt bilayer integrity and to induce mechanical changes in the membrane $[123,124]$. Another factor regulating the interaction of htt with lipid membranes mediated by $\mathrm{Nt}^{17}$ are the previously discussed PTMs, which are known to be involved in htt translocating to specific organelles [39, 125]. Additional affinity-based components for the interaction of other AHs with lipid bilayers also play a role, including electrostatics [126, 127], partitioning of lipid components [128-130], or the presence of

other specific recognition motifs $[131,132]$. The availability of $\mathrm{Nt}^{17}$ to interact with lipid membranes could also play an important role in cell to cell transport of htt aggregates. This may prove particularly important with the realization that there are non-cellautonomous toxic effects associated with HD [133-137]. The study of htt/lipid membrane interaction may contribute to the understanding of the mechanism of pathology of HD.

\subsection{Lipid Composition in huntingtin/lipid interaction}

As previously discussed, htt is found highly associated with a variety of cellular and subcellular membranes that are predominantly comprised of lipids, consisting of physiological composition of cholesterol, sphingomyelin and ganglioside (GM1). Cholesterol affects the functional properties of membrane-resident proteins (ion channels and transmitter receptors), as well as plays a role in signal transduction, synaptogenesis, and neurotransmitter release [138-140]. Abnormalities in cholesterol metabolism and

\section{Introduction}


homeostasis have been observed in cellular and animal models of HD, as well as in HD patient tissues [141-143]. However, the specifics of altered cholesterol content associated with HD remains controversial. It has also been demonstrated that reduced synthesis of a gangliosides, GM1, occurs in the cells and brains of HD patients, leading to increased programmed cell death. However, administration of GM1by continuous infusion into the ventricles of brains of HD mice results in complete reversal of motor defects in these mice [144], and increased in phosphorylation at Ser13 and Ser16 [90], suggesting that the mechanism of GM1 neuroprotection could be phosphorylation of htt. While the importance of htt aggregation in HD has long been appreciated, the impact of specific lipid components, such as cholesterol, GM1 and sphingomyelin, on modulating htt aggregation is poorly understood. The final part of this thesis involves evaluating how exogenously GM1, sphingomyelin, added and cholesterol, affects htt/lipid membranes interaction.

\subsection{Dissertation Outline and Study Rationale}

Although it has been over two decades since the discovery of the IT15 gene, great strides have been made in understanding HD, but there is still no therapeutics that either cures or stops disease progression. Based on this and the ongoing discussion on the role of lipid membranes and posttranslational modification as potential modulators of htt aggregation the following topics are addressed in this dissertation:

(1) A variety of protein motifs play a critical role in sensing and targeting specific physical properties of membranes. Htt, in particular, interacts with a variety of membranous structures within the cell, and $\mathrm{Nt}^{17}$ of htt is an $\mathrm{AH}$ lipid-binding

\section{Introduction}


domain. AHs are also known to detect membrane curvature. Flat supported lipid bilayers are commonly used as model membranes for AFM studies of protein aggregation; however, these supported bilayers usually lack curvature. Chapters 2 and 3 addresses if htt exon1 preferentially binds curved membranes by developing and validating a system of a model supported lipid bilayer with both flat and curved regions. This system is exposed to a truncated htt peptide to determine if curvature plays a role in the interaction of htt with lipid membranes.

(2) $\mathrm{Nt}^{17}$ influences the aggregation of $\mathrm{N}$-terminal fragments of htt and their direct interactions with lipid membranes. Furthermore, PTMs within $\mathrm{Nt}^{17}$ have a profound effect on the toxicity associated with mutant htt containing expanded polyQ domains. However, the impact of acetylation of $\mathrm{Nt}^{17}$ on modulating htt aggregation and its interactions with lipid bilayers is poorly understood. Chapter 4 investigates the influence of acetylation of $\mathrm{Nt}^{17}$ on htt aggregation on lipid bilayers.

(3) Finally, the relevance of htt aggregation in HD has been studied extensively, and lipids play important roles on the aggregation of a variety of htt fragments. However, the role and impact of specific lipid components, such as cholesterol, sphingomyelin and GM1, on modulating htt aggregation is not quite understood. Abnormalities in cholesterol homeostasis having been implicated in HD, and direct administration of GM1 into yeast artificial chromosome (YAC) mice model expressing the full length mutant huntingtin was found to reduce pathology. Chapter 5 sought to understand the role of these specific lipid components on htt aggregation with the potential implications to HD.

\section{Introduction}




\subsection{Atomic Force Microscopy}

To address these questions, a number of techniques including, AFM were used to study htt/lipid interaction. Htt forms a variety of nanoscale aggregates through a complex pathway that involve oligomers, off-pathway aggregates and fibrils (as previously shown in Fig. 1). Due to the complexity of the aggregation pathway and nanoscale size of these aggregates, AFM has emerged as a robust and versatile tool for distinguishing and characterizing the morphology of disease-related protein aggregates [145-147]. AFM can be operated both in air and solution, allowing the dynamic tracking of aggregates under near physiological conditions, at nanoscale resolution [29, 145, 148-152]. Since its inception by Binning and Rohrer [153], AFM has become an invaluable tool for threedimensional imaging as well as measuring structural features of aggregates [124, 154]. As shown in Fig. 3, AFM measures the vertical displacement of a cantilever with an ultra-sharp probe that interacts with the sample surfaces. A laser is reflected off the back of the cantilever into a photosensitive photodiode detector. The tip is brought close to the sample surface, and the interaction tip/sample forces cause the deflection of the cantilever. This deflection is measured using an optical lever provided by reflecting a laser off of the cantilever onto a photodiode. The piezo scanner enables the cantilever to scan back and forth across the sample. Images are generated by raster scanning the tip across the sample surface. The feedback loop helps maintain constant deflection or amplitude while imaging.

There are three main modes of AFM, namely contact, tapping/intermittent, and non-contact mode. In contact mode, commonly described as the most direct AFM mode, the probe is brought in direct contact with the surface and the deflection of the cantilever

\section{Introduction}




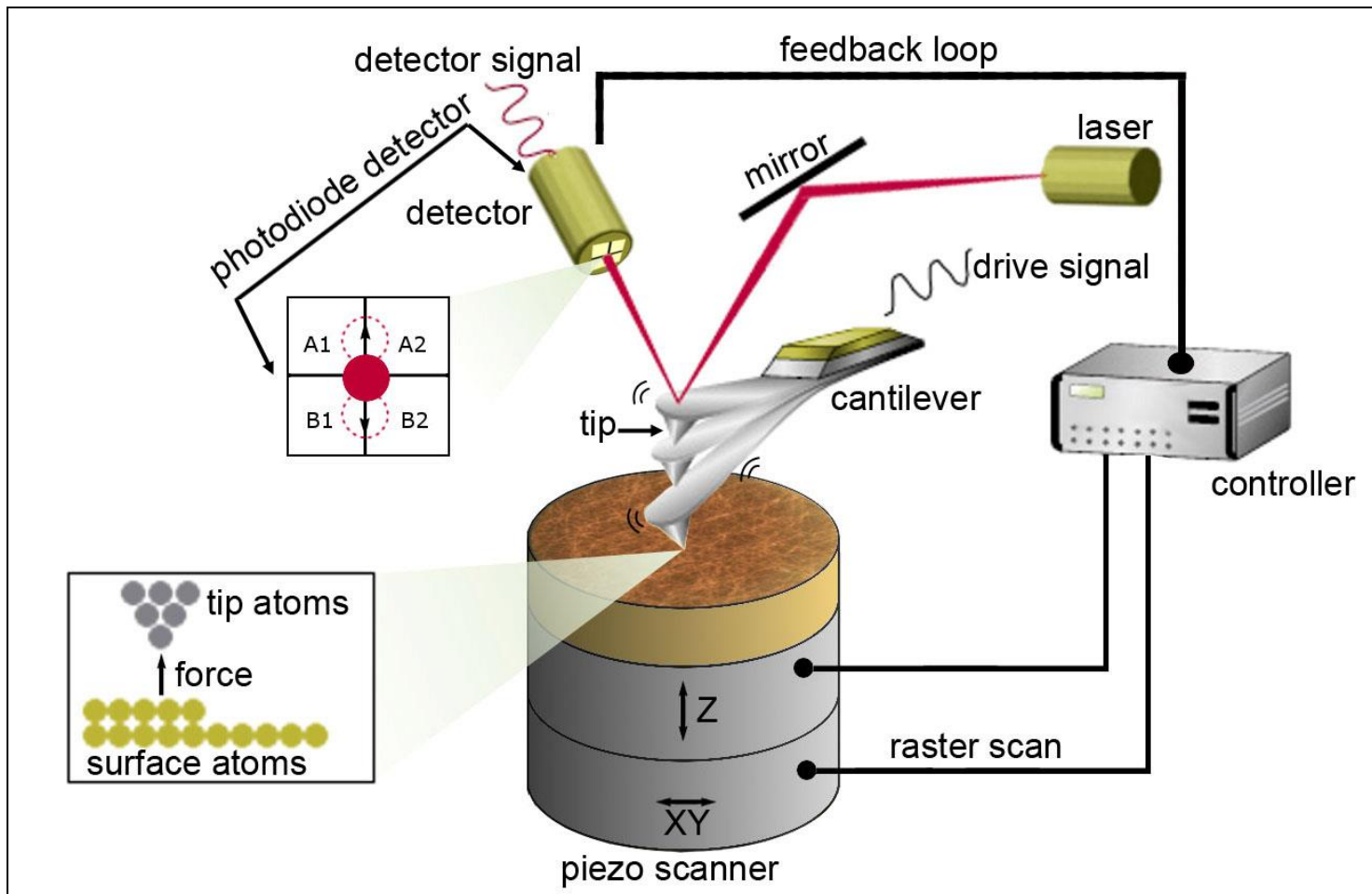

Figure 3. Basic schematic illustration of an AFM. The ultra-sharp tip is brought in close contact with the surface, causing the deflection of the cantilever. The defection is measured by the photodiode, and together with the feedback loop and controller, threedimensional topographical images of surfaces with nanoscale resolution can be obtained

is monitored. This AFM mode allows for fast scanning and is good for imaging hard samples. However, it is heavily influenced by frictional and adhesive forces [155], and as such considerable amount of lateral and shear forces are generated, causing damage to soft , biological samples [156] .

In noncontact AFM, the probe does not "touch" the surface but it oscillates above the surface during scanning. As the tip approaches the surface, the forces between the tip and the surface (as shown in Fig. 3) change the amplitude of the cantilever's resonant vibration. In tapping mode, the cantilever is harmonically oscillated near its resonance frequency, $\omega_{o}$, by a piezoelectric bimorph element mounted near the cantilever root. The probe tip is placed in close proximity to the sample surface and allowed to intermittently 1. Introduction 
contact the sample, leading to a decrease of the cantilever oscillation amplitude from the "free" amplitude, $A_{0}$, to a tapping amplitude, $A[157,158]$. Owing to its ability to minimize potential damage to delicate, biological samples, tapping mode AFM was used for the studies presented in this dissertation.

\subsection{References}

1. Soto, C. and L.D. Estrada, Protein misfolding and neurodegeneration. Archives of Neurology, 2008. 65(2): p. 184-189.

2. Soto, C., Unfolding the role of protein misfolding in neurodegenerative diseases. Nat Rev Neurosci, 2003. 4(1): p. 49-60.

3. Miller, Jason, et al., Identifying polyglutamine protein species in situ that best predict neurodegeneration. Nature chemical biology, 2011. 7(12): p. 925-934.

4. Cleary, J.P., et al., Natural oligomers of the amyloid-protein specifically disrupt cognitive function. Nature Neuroscience, 2005. 8(1): p. 79-84.

5. Kayed, R., et al., Common structure of soluble amyloid oligomers implies common mechanism of pathogenesis. Science, 2003. 300(5618): p. 486-489.

6. Group, T.H.S.D.C.R., A Novel Gene Containing a Trinucleotide Repeat That Is Expanded and Unstable on Huntington's Disease Chromosomes Cell, 1993. 72: p. 971-983.

7. Legleiter, J., et al., Mutant Huntingtin Fragments Form Oligomers in a Polyglutamine Length-dependent Manner in Vitro and in Vivo. Journal of Biological Chemistry, 2010. 285(19): p. 14777-14790.

1. Introduction 
8. Vonsattel, J.P., et al., Neuropathological classification of huntingtons-disease. Journal of Neuropathology and Experimental Neurology, 1985. 44(6): p. 559-577.

9. Poirier, M.A., et al., Huntingtin spheroids and protofibrils as precursors in polyglutamine fibrilization. Journal of Biological Chemistry, 2002. 277(43): p. $41032-41037$.

10. Kim, Y.J., et al., Caspase 3-cleaved N-terminal fragments of wild-type and mutant huntingtin are present in normal and Huntington's disease brains, associate with membranes, and undergo calpain-dependent proteolysis. Proceedings of the National Academy of Sciences, 2001. 98(22): p. 12784-12789.

11. Cooper, J.K., et al., Truncated N-Terminal Fragments of Huntingtin with Expanded Glutamine Repeats form Nuclear and Cytoplasmic Aggregates in Cell Culture. Human Molecular Genetics, 1998. 7(5): p. 783-790.

12. Hoffner, G., M.-L. Island, and P. Djian, Purification of neuronal inclusions of patients with Huntington's disease reveals a broad range of $\mathrm{N}$-terminal fragments of expanded huntingtin and insoluble polymers. Journal of Neurochemistry, 2005. 95(1): p. 125-136.

13. Davies, S.W., et al., Formation of neuronal intranuclear inclusions underlies the neurological dysfunction in mice transgenic for the HD mutation. Cell, 1997. 90(3): p. 537-548.

14. Landles, C., et al., Proteolysis of Mutant Huntingtin Produces an Exon 1 Fragment That Accumulates as an Aggregated Protein in Neuronal Nuclei in Huntington Disease. J. Biol. Chem., 2010. 285(12): p. 8808-8823.

1. Introduction 
15. Sathasivam, K., et al., Aberrant splicing of HTT generates the pathogenic exon 1 protein in Huntington disease. Proceedings of the National Academy of Sciences, 2013. 110(6): p. 2366-2370.

16. DiFiglia, M., et al., Aggregation of Huntingtin in Neuronal Intranuclear Inclusions and Dystrophic Neurites in Brain. Science, 1997. 277(5334): p. 19901993.

17. Ratovitski, T., et al., Mutant Huntingtin N-terminal Fragments of Specific Size Mediate Aggregation and Toxicity in Neuronal Cells. Journal of Biological Chemistry, 2009. 284(16): p. 10855-10867.

18. Penney, J.B., et al., CAG repeat number governs the development rate of pathology in Huntington's disease. Annals of Neurology, 1997. 41(5): p. 689-692.

19. Snell, R.G., et al., Relationship between trinucleotide repeat expansion and phenotypic variation in huntingtons-disease. Nature Genetics, 1993. 4(4): p. 393397.

20. Tobin, A.J. and E.R. Signer, Huntington's disease: the challenge for cell biologists. Trends in Cell Biology, 2000. 10(12): p. 531-536.

21. Sturrock, A. and B.R. Leavitt, The clinical and genetic features of Huntington disease. Journal of Geriatric Psychiatry and Neurology, 2010: p. 0891988710383573.

22. Scherzinger, E., et al., Huntingtin-encoded polyglutamine expansions form amyloid-like protein aggregates in vitro and in vivo. Cell, 1997. 90(3): p. 549558.

1. Introduction 
23. Scherzinger, E., et al., Self-assembly of polyglutamine-containing huntingtin fragments into amyloid-like fibrils: Implications for Huntington's disease pathology. Proceedings of the National Academy of Sciences of the United States of America, 1999. 96(8): p. 4604-4609.

24. Wetzel, R., Physical Chemistry of Polyglutamine: Intriguing Tales of a Monotonous Sequence. Journal of Molecular Biology, 2012. 421(4-5): p. 466490.

25. Lunkes, A. and J.L. Mandel, A cellular model that recapitulates major pathogenic steps of Huntington's disease. Human Molecular Genetics, 1998. 7(9): p. 13551361.

26. Saudou, F., et al., Huntingtin acts in the nucleus to induce apoptosis but death does not correlate with the formation of intranuclear inclusions. Cell, 1998. 95(1): p. 55-66.

27. Hackam, A.S., et al., The influence of Huntingtin protein size on nuclear localization and cellular toxicity. Journal of Cell Biology, 1998. 141(5): p. 10971105.

28. Tanaka, M., et al., Intra- and intermolecular beta-pleated sheet formation in glutamine-repeat inserted myoglobin as a model for polyglutamine diseases. Journal of Biological Chemistry, 2001. 276(48): p. 45470-45475.

29. Wacker, J.L., et al., Hsp70 and Hsp40 attenuate formation of spherical and annular polyglutamine oligomers by partitioning monomer. Nature Structural \& Molecular Biology, 2004. 11(12): p. 1215-1222.

1. Introduction 
30. Nucifora, L.G., et al., Identification of Novel Potentially Toxic Oligomers Formed in Vitro from Mammalian-derived Expanded huntingtin Exon-1 Protein. Journal of Biological Chemistry, 2012. 287(19): p. 16017-16028.

31. Legleiter, J., et al., Monoclonal Antibodies Recognize Distinct Conformational Epitopes Formed by Polyglutamine in a Mutant Huntingtin Fragment. Journal of Biological Chemistry, 2009. 284(32): p. 21647-21658.

32. Chen, S., et al., Polyglutamine aggregation behavior in vitro supports a recruitment mechanism of cytotoxicity. Journal of Molecular Biology, 2001. 311(1): p. 173-182.

33. Chen, S.M., et al., Amyloid-like features of polyglutamine aggregates and their assembly kinetics. Biochemistry, 2002. 41(23): p. 7391-7399.

34. Bernacki, J.P. and R.M. Murphy, Model Discrimination and Mechanistic Interpretation of Kinetic Data in Protein Aggregation Studies. Biophysical Journal, 2009. 96(7): p. 2871-2887.

35. Walters, R.H. and R.M. Murphy, Examining Polyglutamine Peptide Length: A Connection between Collapsed Conformations and Increased Aggregation. Journal of Molecular Biology, 2009. 393(4): p. 978-992.

36. Walters, R.H. and R.M. Murphy, Aggregation Kinetics of Interrupted Polyglutamine Peptides. Journal of Molecular Biology, 2011. 412(3): p. 505-519.

37. Arrasate, M., et al., Inclusion body formation reduces levels of mutant huntingtin and the risk of neuronal death. Nature, 2004. 431(7010): p. 805-810.

1. Introduction 
38. Gutekunst, C.A., et al., Nuclear and neuropil aggregates in Huntington's disease: Relationship to neuropathology. Journal of Neuroscience, 1999. 19(7): p. 25222534.

39. Atwal, R.S., et al., Huntingtin has a membrane association signal that can modulate huntingtin aggregation, nuclear entry and toxicity. Human Molecular Genetics, 2007. 16(21): p. 2600-2615.

40. Kegel-Gleason, K.B., Huntingtin Interactions with Membrane Phospholipids: Strategic Targets for Therapeutic Intervention? Journal of Huntington's Disease, 2013. 2(3): p. 239-250.

41. Leitman, J., F. Ulrich Hartl, and G.Z. Lederkremer, Soluble forms of polyQexpanded huntingtin rather than large aggregates cause endoplasmic reticulum stress. Nat Commun, 2013. 4.

42. Crick, S.L., et al., Unmasking the roles of $\mathrm{N}$ - and $\mathrm{C}$-terminal flanking sequences from exon 1 of huntingtin as modulators of polyglutamine aggregation. Proceedings of the National Academy of Sciences, 2013. 110(50): p. 2007520080.

43. Mishra, R., et al., Serine Phosphorylation Suppresses Huntingtin Amyloid Accumulation by Altering Protein Aggregation Properties. Journal of Molecular Biology, 2012. 424(1-2): p. 1-14.

44. Sivanandam, V.N., et al., The Aggregation-Enhancing Huntingtin N-Terminus Is Helical in Amyloid Fibrils. Journal of the American Chemical Society, 2011. 133(12): p. 4558-4566.

1. Introduction 
45. Kelley, N.W., et al., The Predicted Structure of the Headpiece of the Huntingtin Protein and Its Implications on Huntingtin Aggregation. Journal of Molecular Biology, 2009. 388(5): p. 919-927.

46. Williamson, T.E., et al., Modulation of Polyglutamine Conformations and Dimer Formation by the N-Terminus of Huntingtin. Journal of Molecular Biology, 2010. 396(5): p. 1295-1309.

47. Thakur, A.K., et al., Polyglutamine disruption of the huntingtin exon $1 \mathrm{~N}$ terminus triggers a complex aggregation mechanism. Nature Structural \& Molecular Biology, 2009. 16(4): p. 380-389.

48. Vitalis, A. and R.V. Pappu, Assessing the contribution of heterogeneous distributions of oligomers to aggregation mechanisms of polyglutamine peptides. Biophysical Chemistry, 2011. 159(1): p. 14-23.

49. Jayaraman, M., et al., Slow Amyloid Nucleation via alpha-Helix-Rich Oligomeric Intermediates in Short Polyglutamine-Containing Huntingtin Fragments. Journal of Molecular Biology, 2012. 415(5): p. 881-899.

50. Olshina, M.A., et al., Tracking Mutant Huntingtin Aggregation Kinetics in Cells Reveals Three Major Populations That Include an Invariant Oligomer Pool. Journal of Biological Chemistry, 2010. 285(28): p. 21807-21816.

51. Jayaraman, M., et al., Kinetically Competing Huntingtin Aggregation Pathways Control Amyloid Polymorphism and Properties. Biochemistry, 2012. 51(13): p. 2706-2716.

1. Introduction 
52. Mishra, R., et al., Inhibiting the Nucleation of Amyloid Structure in a Huntingtin Fragment by Targeting alpha-Helix-Rich Oligomeric Intermediates. Journal of Molecular Biology, 2012. 415(5): p. 900-917.

53. Burke, K.A., et al., The Interaction of Polyglutamine Peptides With Lipid Membranes is Regulated by Flanking Sequences Associated with Huntingtin. Journal of Biological Chemistry, 2013.

54. Burke, K.A., J. Godbey, and J. Legleiter, Assessing mutant huntingtin fragment and polyglutamine aggregation by atomic force microscopy. Methods, 2011. 53(3): p. 275-284.

55. Sahoo, B., et al., Aggregation Behavior of Chemically Synthesized, Full-Length Huntingtin Exon1. Biochemistry, 2014. 53(24): p. 3897-3907.

56. Drin, G. and B. Antonny, Amphipathic helices and membrane curvature. FEBS Letters, 2010. 584(9): p. 1840-1847.

57. Brass, V., et al., An Amino-terminal Amphipathic $\alpha$-Helix Mediates Membrane Association of the Hepatitis C Virus Nonstructural Protein 5A. Journal of Biological Chemistry, 2002. 277(10): p. 8130-8139.

58. Georgieva, Elka R., et al., Tau Binds to Lipid Membrane Surfaces via Short Amphipathic Helices Located in Its Microtubule-Binding Repeats. Biophysical Journal, 2014. 107(6): p. 1441-1452.

59. Cui, H., E. Lyman, and G.A. Voth, Mechanism of Membrane Curvature Sensing by Amphipathic Helix Containing Proteins. Biophysical Journal, 2011. 100(5): p. 1271-1279.

1. Introduction 
60. Chaibva, M., K.A. Burke, and J. Legleiter, Curvature Enhances Binding and Aggregation of Huntingtin at Lipid Membranes. Biochemistry, 2014. 53(14): p. $2355-2365$.

61. Gouttenoire, J., et al., Amphipathic $\alpha$-helix AH2 is a major determinant for the oligomerization of hepatitis $C$ virus nonstructural protein $4 B$. Journal of virology, 2010. 84(24): p. 12529-12537.

62. Westerlund, J.A. and K.H. Weisgraber, Discrete carboxyl-terminal segments of apolipoprotein E mediate lipoprotein association and protein oligomerization. Journal of Biological Chemistry, 1993. 268(21): p. 15745-50.

63. Nagarajan, A., S. Jawahery, and S. Matysiak, The Effects of Flanking Sequences in the Interaction of Polyglutamine Peptides with a Membrane Bilayer. The Journal of Physical Chemistry B, 2013. 118(24): p. 6368-6379

64. Arndt, J.A., et al., Lysine Residues in the N-Terminal Huntingtin Amphipathic $\alpha$ Helix Play a Key Role in Peptide Aggregation. . Journal of Mass Spectrometry 2015. 50(1): p. 117-126

65. Bugg, C.W., et al., Structural Features and Domain Organization of Huntingtin Fibrils. Journal of Biological Chemistry, 2012. 287(38): p. 31739-31746.

66. Colby, D.W., et al., Potent inhibition of huntingtin aggregation and cytotoxicity by a disulfide bond-free single-domain intracellular antibody. Proceedings of the National Academy of Sciences of the United States of America, 2004. 101(51): p. 17616-17621.

1. Introduction 
67. Colby, D.W., et al., Development of a Human Light Chain Variable Domain (VL) Intracellular Antibody Specific for the Amino Terminus of Huntingtin via Yeast Surface Display. Journal of Molecular Biology, 2004. 342(3): p. 901-912.

68. Tam, S., et al., The chaperonin TRiC controls polyglutamine aggregation and toxicity through subunit-specific interactions. Nature cell biology, 2006. 8(10): p. $1155-1162$.

69. Tam, S., et al., The chaperonin TRiC blocks a huntingtin sequence element that promotes the conformational switch to aggregation. Nature Structural \& Molecular Biology, 2009. 16(12): p. 1279-U98.

70. McLear, J.A., et al., Combinational approach of intrabody with enhanced Hsp70 expression addresses multiple pathologies in a fly model of Huntington's disease. Faseb Journal, 2008. 22(6): p. 2003-2011.

71. Wolfgang, W.J., et al., Suppression of Huntington's disease pathology in Drosophila by human single-chain Fv antibodies. Proceedings of the National Academy of Sciences of the United States of America, 2005. 102(32): p. 1156311568.

72. Wacker, J.L., et al., Loss of Hsp70 Exacerbates Pathogenesis But Not Levels of Fibrillar Aggregates in a Mouse Model of Huntington's Disease. Journal of Neuroscience, 2009. 29(28): p. 9104-9114.

73. Lotz, G.P., et al., Hsp70 and Hsp40 Functionally Interact with Soluble Mutant Huntingtin Oligomers in a Classic ATP-dependent Reaction Cycle. Journal of Biological Chemistry, 2010. 285(49): p. 38183-38193.

1. Introduction 
74. Monsellier, E., et al., Molecular Interaction between the Chaperone Hsc70 and the N-terminal Flank of Huntingtin Exon 1 Modulates Aggregation. Journal of Biological Chemistry, 2014. 290(5): p. 2560-2576.

75. Choudhury, K.R. and N.P. Bhattacharyya, Chaperone protein HYPK interacts with the first 17 amino acid region of Huntingtin and modulates mutant HTTmediated aggregation and cytotoxicity. Biochemical and biophysical research communications, 2015. 456(1): p. 66-73.

76. $\mathrm{Li}, \mathrm{P}$, et al., The structure of a polyQ-anti-polyQ complex reveals binding according to a linear lattice model. Nature Structural \& Molecular Biology, 2007. 14(5): p. 381-387.

77. Ko, J., S. Ou, and P.H. Patterson, New anti-huntingtin monoclonal antibodies: Implications for huntingtin conformation and its binding proteins. Brain Research Bulletin, 2001. 56(3-4): p. 319-329.

78. Trottier, Y., et al., Polyglutamine expansion as a pathological epitope in huntingtons-disease and 4 dominant cerebellar ataxias. Nature, 1995. 378(6555): p. 403-406.

79. Bennett, M.J., et al., A linear lattice model for polyglutamine in CAG-expansion diseases. Proceedings of the National Academy of Sciences of the United States of America, 2002. 99(18): p. 11634-11639.

80. Lecerf, J.M., et al., Human single-chain Fv intrabodies counteract in situ huntingtin aggregation in cellular models of Huntington's disease. Proceedings of the National Academy of Sciences of the United States of America, 2001. 98(8): p. $4764-4769$.

1. Introduction 
81. Steffan, J.S., et al., SUMO modification of Huntingtin and Huntington's disease pathology. Science, 2004. 304(5667): p. 100-104.

82. Pennuto, M., I. Palazzolo, and A. Poletti, Post-translational modifications of expanded polyglutamine proteins: impact on neurotoxicity. Human Molecular Genetics, 2009. 18: p. R40-R47.

83. Ehrnhoefer, D.E., L. Sutton, and M.R. Hayden, Small changes, big impact: posttranslational modifications and function of huntingtin in Huntington disease. Neuroscientist, 2011. 17(5): p. 475-92.

84. Dorval, V. and P.E. Fraser, SUMO on the road to neurodegeneration. Biochimica et Biophysica Acta (BBA)-Molecular Cell Research, 2007. 1773(6): p. 694-706.

85. Díaz-Hernández, M., et al., Inhibition of $26 S$ proteasome activity by huntingtin filaments but not inclusion bodies isolated from mouse and human brain. Journal of neurochemistry, 2006. 98(5): p. 1585-1596.

86. Borrell-Pages, M., et al., Huntington's disease: from huntingtin function and dysfunction to therapeutic strategies. Cellular and Molecular Life Sciences, 2006. 63(22): p. 2642-2660.

87. O'Rourke, J.G., et al., SUMO-2 and PIAS1 Modulate Insoluble Mutant Huntingtin Protein Accumulation. Cell Reports, 2013. 4(2): p. 362-375.

88. Hannoun, Z., et al., Post-translational modification by SUMO. Toxicology, 2010. 278(3): p. 288-293.

89. Aiken, C.T., et al., Phosphorylation of Threonine 3 Implications for huntingtin aggregation and neurotoxicity. Journal of Biological Chemistry, 2009. 284(43): p. 29427-29436.

1. Introduction 
90. Di Pardo, A., et al., Ganglioside GM1 induces phosphorylation of mutant huntingtin and restores normal motor behavior in Huntington disease mice. Proceedings of the National Academy of Sciences of the United States of America, 2012. 109(9): p. 3528-3533.

91. Thompson, L.M., et al., IKK phosphorylates Huntingtin and targets it for degradation by the proteasome and lysosome. Journal of Cell Biology, 2009. 187(7): p. 1083-1099.

92. Gu, X.F., et al., Serines 13 and 16 Are Critical Determinants of Full-Length Human Mutant Huntingtin Induced Disease Pathogenesis in HD Mice. Neuron, 2009. 64(6): p. 828-840.

93. Jeong, H., et al., Acetylation Targets Mutant Huntingtin to Autophagosomes for Degradation. Cell, 2009. 137(1): p. 60-72.

94. Kalchman, M.A., et al., Huntingtin is ubiquitinated and interacts with a specific ubiquitin-conjugating enzyme. Journal of Biological Chemistry, 1996. 271(32): p. $19385-19394$.

95. Yanai, A., et al., Palmitoylation of huntingtin by HIP14is essential for its trafficking and function. Nat Neurosci, 2006. 9(6): p. 824-831.

96. Kahlem, P., H. Green, and P. Djian, Transglutaminase action imitates Huntington's disease: Selective polymerization of huntingtin containing expanded polyglutamine. Molecular Cell, 1998. 1(4): p. 595-601.

97. Warby, S.C., et al., Huntingtin phosphorylation on serine 421 is significantly reduced in the striatum and by polyglutamine expansion in vivo. Journal of Neurology Neurosurgery and Psychiatry, 2005. 14(11): p. 1569-1577

1. Introduction 
98. Humbert, S. and F. Saudou, Huntingtin phosphorylation and signaling pathways that regulate toxicity in Huntington's disease. Clinical Neuroscience Research, 2003. 3(3): p. 149-155.

99. Watkin, E.E., et al., Phosphorylation of Mutant Huntingtin at Serine 116 Modulates Neuronal Toxicity. Plos One, 2014. 9(2): p. e88284

100. Atwal, R.S., et al., Kinase inhibitors modulate huntingtin cell localization and toxicity. Nat Chem Biol, 2011. 7(7): p. 453-460.

101. Subramaniam, S., et al., Rhes, a Striatal Specific Protein, Mediates MutantHuntingtin Cytotoxicity. Science, 2009. 324(5932): p. 1327-1330.

102. Jana, N.R., et al., Co-chaperone CHIP associates with expanded polyglutamine protein and promotes their degradation by proteasomes. Journal of Biological Chemistry, 2005. 280(12): p. 11635-11640.

103. Cong, X., et al., Mass spectrometric identification of novel lysine acetylation sites in huntingtin. Molecular \& Cellular Proteomics, 2011. 10(10): p. M111. 009829.

104. Gunawardena, S., et al., Disruption of Axonal Transport by Loss of Huntingtin or Expression of Pathogenic PolyQ Proteins in Drosophila. Neuron, 2003. 40(1): p. $25-40$.

105. Gauthier, L.R., et al., Huntingtin controls neurotrophic support and survival of neurons by enhancing BDNF vesicular transport along microtubules. Cell, 2004. 118(1): p. 127-138.

106. Lee, W.-C.M., M. Yoshihara, and J.T. Littleton, Cytoplasmic aggregates trap polyglutamine-containing proteins and block axonal transport in a Drosophila

1. Introduction 
model of Huntington's disease. Proceedings of the National Academy of Sciences of the United States of America, 2004. 101(9): p. 3224-3229.

107. Pal, A., et al., Huntingtin-HAP40 complex is a novel Rab5 effector that regulates early endosome motility and is up-regulated in Huntington's disease. The Journal of Cell Biology, 2006. 172(4): p. 605-618.

108. Kegel, K.B., et al., Huntingtin expression stimulates endosomal-lysosomal activity, endosome tubulation, and autophagy. The Journal of neuroscience, 2000. 20(19): p. 7268-7278.

109. Suopanki, J., et al., Interaction of huntingtin fragments with brain membranes clues to early dysfunction in Huntington's disease. Journal of Neurochemistry, 2006. 96(3): p. 870-884.

110. Persichetti, F., et al., Mutant Huntingtin Forms in Vivo Complexes with Distinct Context-Dependent Conformations of the Polyglutamine Segment. Neurobiology of Disease, 1999. 6(5): p. 364-375.

111. Zheng, Z., et al., An N-terminal Nuclear Export Signal Regulates Trafficking and Aggregation of Huntingtin (Htt) Protein Exon 1. Journal of Biological Chemistry, 2013. 288(9): p. 6063-6071.

112. Kegel, K.B., et al., Huntingtin associates with acidic phospholipids at the plasma membrane. Journal of Biological Chemistry, 2005. 280(43): p. 36464-36473.

113. Suopanki, J., et al., Interaction of huntingtin fragments with brain membranes clues to early dysfunction in Huntington's disease. Journal of Neurochemistry, 2006. 96(3): p. 870-884.

1. Introduction 
114. Strehlow, A.N.T., J.Z. Li, and R.M. Myers, Wild-type huntingtin participates in protein trafficking between the Golgi and the extracellular space. Human Molecular Genetics, 2007. 16(4): p. 391-409.

115. Myre, M.A., et al., Deficiency of Huntingtin Has Pleiotropic Effects in the Social Amoeba <italic>Dictyostelium discoideum</italic>. PLoS Genet, 2011. 7(4): p. e1002052.

116. Ritch, J.J., et al., Multiple phenotypes in Huntington disease mouse neural stem cells. Molecular and Cellular Neuroscience, 2012. 50(1): p. 70-81.

117. Jacobsen, J.C., et al., HD CAG-correlated gene expression changes support a simple dominant gain of function. Human Molecular Genetics, 2011. 20(14): p. 2846-2860.

118. Caviston, J.P. and E.L.F. Holzbaur, Huntingtin as an essential integrator of intracellular vesicular trafficking. Trends in Cell Biology, 2009. 19(4): p. 147155.

119. Rockabrand, E., et al., The first 17 amino acids of Huntingtin modulate its subcellular localization, aggregation and effects on calcium homeostasis. Human Molecular Genetics, 2007. 16(1): p. 61-77.

120. Kegel, K.B., et al., Huntingtin associates with acidic phospholipids at the plasma membrane. Journal of Biological Chemistry, 2005. 280(43): p. 36464-36473.

121. Michalek, M., Evgeniy S. Salnikov, and B. Bechinger, Structure and Topology of the Huntingtin 117 Membrane Anchor by a Combined Solution and Solid-State NMR Approach. Biophysical Journal, 2013. 105(3): p. 699-710.

1. Introduction 
122. Caron, N.S., et al., Polyglutamine domain flexibility mediates the proximity between flanking sequences in huntingtin. Proceedings of the National Academy of Sciences, 2013. 110(36): p. 14610-14615.

123. Burke, K.A., et al., Huntingtin disrupts lipid bilayers in a polyQ-length dependent manner. Biochimica et Biophysica Acta (BBA) - Biomembranes, 2013. 1828(8): p. $1953-1961$.

124. Burke, K.A., E.A. Yates, and J. Legleiter, Amyloid-Forming Proteins Alter the Local Mechanical Properties of Lipid Membranes. Biochemistry, 2013. 52(5): p. 808-817.

125. Xia, J.R., et al., Huntingtin contains a highly conserved nuclear export signal. Human Molecular Genetics, 2003. 12(12): p. 1393-1403.

126. McLaughlin, S. and D. Murray, Plasma membrane phosphoinositide organization by protein electrostatics. Nature, 2005. 438(7068): p. 605-611.

127. Yeung, T., et al., Membrane phosphatidylserine regulates surface charge and protein localization. Science, 2008. 319(5860): p. 210-213.

128. Linder, M.E. and R.J. Deschenes, Palmitoylation: policing protein stability and traffic. Nature Reviews Molecular Cell Biology, 2007. 8(1): p. 74-84.

129. Manneville, J.-B., et al., COPI coat assembly occurs on liquid-disordered domains and the associated membrane deformations are limited by membrane tension. Proceedings of the National Academy of Sciences of the United States of America, 2008. 105(44): p. 16946-16951.

130. Rocks, O., et al., An acylation cycle regulates localization and activity of palmitoylated Ras isoforms. Science, 2005. 307(5716): p. 1746-1752.

1. Introduction 
131. Lemmon, M.A., Membrane recognition by phospholipid-binding domains. Nature Reviews Molecular Cell Biology, 2008. 9(2): p. 99-111.

132. McMahon, H.T. and J.L. Gallop, Membrane curvature and mechanisms of dynamic cell membrane remodelling. Nature, 2005. 438(7068): p. 590-596.

133. Lobsiger, C.S. and D.W. Cleveland, Glial cells as intrinsic components of noncell-autonomous neurodegenerative disease. Nat Neurosci, 2007. 10(11): p. 13551360.

134. Giorgini, F., et al., A genomic screen in yeast implicates kynurenine 3monooxygenase as a therapeutic target for Huntington disease. Nature Genetics, 2005. 37(5): p. 526-531.

135. Yang, W., et al., Aggregated polyglutamine peptides delivered to nuclei are toxic to mammalian cells. Human Molecular Genetics, 2002. 11(23): p. 2905-2917.

136. Ren, P.-H., et al., Cytoplasmic penetration and persistent infection of mammalian cells by polyglutamine aggregates. Nature Cell Biology, 2009. 11(2): p. 219$\mathrm{U} 232$.

137. Cicchetti, F., et al., Neural transplants in patients with Huntington's disease undergo disease-like neuronal degeneration. Proceedings of the National Academy of Sciences, 2009. 106(30): p. 12483-12488.

138. Burger, K., G. Gimpl, and F. Fahrenholz, Regulation of receptor function by cholesterol. Cellular and Molecular Life Sciences, 2000. 57(11): p. 1577-1592.

139. Thiele, C., et al., Cholesterol binds to synaptophysin and is required for biogenesis of synaptic vesicles. Nature Cell Biology, 2000. 2(1): p. 42-49.

1. Introduction 
140. Mauch, D.H., et al., CNS synaptogenesis promoted by glia-derived cholesterol. Science, 2001. 294(5545): p. 1354-1357.

141. Valenza, M. and E. Cattaneo, Emerging roles for cholesterol in Huntington's disease. Trends in Neurosciences, 2011. 34(9): p. 474-486.

142. Karasinska, J.M. and M.R. Hayden, Cholesterol metabolism in Huntington disease. Nat Rev Neurol, 2011. 7(10): p. 561-572.

143. Leoni, V. and C. Caccia, The impairment of cholesterol metabolism in Huntington disease. Biochimica et Biophysica Acta (BBA) - Molecular and Cell Biology of Lipids, 2015. 1851(8): p. 1095-1105

144. Maglione, V., et al., Impaired ganglioside metabolism in Huntington's disease and neuroprotective role of GM1. The Journal of Neuroscience, 2010. 30(11): p. 4072-4080.

145. Blackley, H.K.L., et al., Morphological development of beta(1-40) amyloid fibrils. Experimental Neurology, 1999. 158(2): p. 437-443.

146. Blackley, H.K.L., et al., In-situ atomic force microscopy study of beta-amyloid fibrillization. Journal of Molecular Biology, 2000. 298(5): p. 833-840.

147. Kowalewski, T. and D.M. Holtzman, In situ atomic force microscopy study of Alzheimer's beta-amyloid peptide on different substrates: New insights into mechanism of beta-sheet formation. Proceedings of the National Academy of Sciences of the United States of America, 1999. 96(7): p. 3688-3693.

148. Legleiter, J., et al., Effect of different anti-A beta antibodies on A beta fibrillogenesis as assessed by atomic force microscopy. Journal of Molecular Biology, 2004. 335(4): p. 997-1006.

1. Introduction 
149. Legleiter, J., et al., Static and dynamic AFM studies of beta-amyloid aggregates and their interaction with relevant biological macromolecules. Neurobiology of Aging, 2004. 25: p. S164-S164.

150. Legleiter, J., et al., In situ AFM studies of astrocyte-secreted apolipoprotein Eand J-containing lipoproteins. Journal of Colloid and Interface Science, 2004. 278(1): p. 96-106.

151. Legleiter, J. and T. Kowalewski, Atomic force microscopy of beta-amyloid: static and dynamic studies of nanostructure and its formation. Methods in molecular biology (Clifton, N.J.), 2004. 242: p. 349-64.

152. Legleiter, J.A., et al., Static and dynamic AFM studies of the effect of anti-A beta antibodies on A beta aggregates and their formation. Biophysical Journal, 2004. 86(1): p. 152A-152A.

153. Binnig, G., C.F. Quate, and C. Gerber, Atomic force microscope. physical Review Letters, 1986. 56(9): p. 930-933.

154. Burke, K.A., E.A. Yates, and J. Legleiter, Biophysical insights into how surfaces, including lipid membranes, modulate protein aggregation related to neurodegeneration. Frontiers in neurology, 2013. 4: p. 17-17.

155. Sagui, C. and T.A. Darden, Molecular dynamics simulations of biomolecules: long-range electrostatic effects. Annual review of biophysics and biomolecular structure, 1999. 28(1): p. 155-179.

156. Hansma, H.G. and J.H. Hoh, Biomolecular imaging with the atomic force microscope. Annual review of biophysics and biomolecular structure, 1994. 23(1): p. 115-140.

1. Introduction 
157. Burnham, N.A., et al., How does a tip tap? Nanotechnology, 1997. 8(2): p. 67-75.

158. Nony, L., R. Boisgard, and J.P. Aime, Nonlinear dynamical properties of an oscillating tip-cantilever system in the tapping mode. Journal of Chemical Physics, 1999. 111(4): p. 1615-1627.

1. Introduction 


\section{Scanning probe acceleration microscope and the development of supported lipid bilayers with curvature ${ }^{1,2}$}

\section{Abstract}

Tapping mode atomic force microscopy (TMAFM) has become a useful method to obtain physical insights into the formation of toxic protein aggregates associated with amyloid diseases on membranes. However, recent advances in reconstructing the timeresolved tip/sample force during the imaging process have provided access to information relating to the mechanical properties of these surfaces. The ability to directly image and measure changes in mechanical properties of lipid bilayers associated with exposure to amyloid-forming peptides in a "quasi" real-time fashion can provide new insights that are exceedingly difficult to obtain using other traditional methods. However, the absence of membrane curvature, which can heavily influence the interaction of proteins at lipid interfaces, is one of the several limitations associated with using flat, supported bilayers as model surfaces. I, therefore, developed model lipid bilayer systems in which the underlying solid support is comprised of flat and curved regions to induce regions of curvature in the bilayer. To accomplish this, $50 \mathrm{~nm}$ silica nanoparticles were deposited on a silicon substrate coated with poly-L-lysine. This substrate was exposed to total brain lipid extract (TBLE) vesicles, which can fuse on surfaces to form lipid bilayers. Using an

AFM based, force reconstruction technique; scanning probe acceleration microscopy

\footnotetext{
This chapter is based on the works:

${ }^{1}$ Recovering Time-Resolved Imaging Forces in Solution by Scanning Probe Acceleration Microscopy: Theory and Application in Surface Science Tools for Nanomaterials Characterization.

${ }^{2}$ Curvature enhances binding and aggregation of huntingtin at lipid membranes.
} 
(SPAM), to measure changes in the mechanical properties of the lipid bilayers, the formation of supported bilayer on the surface that contained flat and curved regions was confirmed. This chapter gives a detailed description of the principles of the SPAM technique, and its application in developing supported lipid bilayers with curvature.

\subsection{Introduction}

TMAFM, [1] a widely used dynamic scanning probe technique, obtains topography images of surfaces by monitoring the oscillation amplitude of a cantilever integrated with an ultrasharp probe tip. In this imaging mode, the cantilever is commonly driven near its resonance frequency, $\omega_{o}$, by a piezoelectric bimorph element mounted near the cantilever root. The probe tip is placed in close proximity to the sample surface and allowed to intermittently contact the sample, leading to a decrease of the cantilever oscillation amplitude from the "free" amplitude, $A_{\mathrm{o}}$, to a tapping amplitude, $A[2,3]$. For rigid surfaces, the cantilever oscillation amplitude decreases linearly as the distance between the tip and the sample, $D_{\mathrm{o}}$, becomes smaller. As a result, surface topography is mapped by raster scanning the probe across the surface while using a feedback loop to continuously adjust the vertical (in the $z$ direction) extension of a piezoelectric scanner to maintain a constant tapping amplitude or set-point ratio, $s=A / A_{\mathrm{o}}$.

There is significant interest in using TMAFM to simultaneously map topography and mechanical properties of surfaces and a variety of methods have been developed toward achieving this goal. While monitoring the phase of the cantilever in tapping mode is a common method to acquire some insight into the mechanical properties of surfaces, $[4,5]$ such images can be difficult to interpret due to various causes of energy dissipation, including capillary forces, [6] viscoelasticity of the sample, [7] cross talk with

2. 
topography, [8] and frictional forces associated with the tilt of the cantilever and/or surface [9]. Furthermore, the phase behavior of the cantilever becomes increasingly complicated when imaging in solution. For example, it has been demonstrated that the phase contrast associated with soft cantilevers imaging in solution can arise from either (i) energy propagation during the tapping event that excites higher eigenmodes of the cantilever mediated by conservative short-range interactions, or (ii) tip/sample energy dissipation [10].

A more straight-forward way to gain insight into sample mechanics is by obtaining the time-resolved force interaction between the surface and the tip during standard TMAFM operation. The first successful reconstruction of time-resolved tip/sample forces in a TMAFM experiment was accomplished by taking the inverse Fourier transform of the Fourier-transformed cantilever trajectory divided by its transfer function [11]. Since then, a variety of other methods have been developed that are capable of obtaining time-resolved tip/sample forces and construct images based on these forces [12-19].

There are several experimental advantages to using tapping forces as a method to map mechanical properties of surfaces. Due to their basis in the tapping mode, such methods offer high spatial resolution and are relatively nondestructive. Other traditional AFM techniques used to measure and/or map mechanical properties of surfaces (i.e., force volume imaging and nanoindentation) often require large deformation of soft samples, leading to decreased spatial resolution and possibly irreversible sample damage, whereas tapping mode forces are adaptive to changes in surfaces properties. As scan rates used for simple tapping mode AFM imaging are typically much faster than other methods 
(a) image lipid bilayer patch on mica and acquire tip deflection trajectory

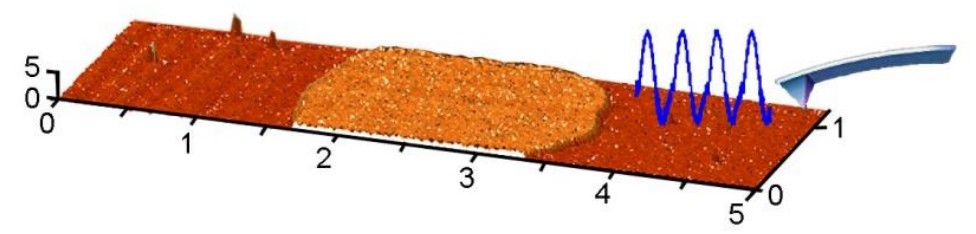

(b)

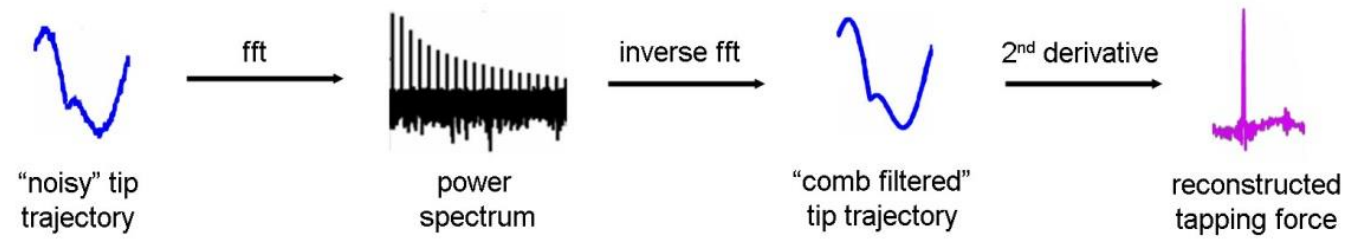

(c) construct maximum tapping force image and correlate tapping force with surface properties
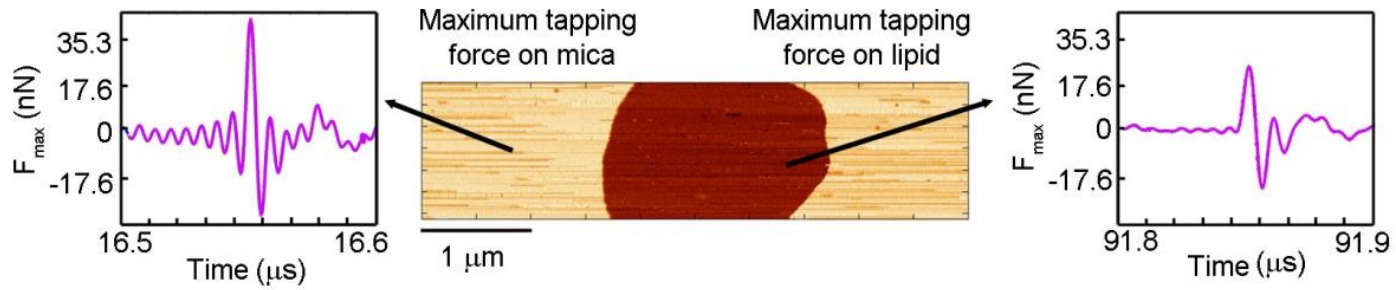

Figure 1. SPAM is a method for reconstructing the time-resolved tip/sample forces associated with TMAFM imaging in solution. In this example, (a) a mica substrate with small supported lipid bilayer patch is imaged to acquire the surface topography while the entire cantilever deflection signal is captured. (b) The deflection trajectory of the cantilever is filtered using a "comb-filter" based on Fourier transforms, and the second derivative of the trajectory is used to reconstruct the tip/sample forces. (c) Images are constructed based on specific features of the tip/sample force, such as the maximum tapping force, which can be related to surface mechanical properties.

, the mapping of surface properties can be accomplished in a timelier manner. This, perhaps, could provide the ability to probe dynamic changes in a surface's mechanical properties in response to some external stimulus or environmental change.

The laboratory of Dr. Tomasz Kowalewski at Carnegie Mellon University, developed a method to recover the time-resolved tip/sample force in TMAFM while operated in aqueous solution is scanning probe acceleration microscopy (SPAM) (Fig. 1) [14]. The underlying principle behind SPAM is that the cantilever in TMAFM acts as an accelerometer, which can be used to extract tip-sample forces during standard tapping mode operation by calculating the second derivative of the cantilever deflection signal. 
Real deflection signals from TMAFM experiments contain noise that is amplified by this analysis. As the signal to noise ratio in most commercially available AFMs would overcome the ability to reconstruct the tapping force by this simple method, a means to filter these signals is crucial if SPAM is to be useful experimentally. This problem is solved by taking advantage of the fact that most of the information about the deflection trajectory and the tapping event is contained in higher harmonic frequencies associated with the drive, $[11,20,21]$ making it possible to filter the signal by "comb" filtering, i.e., by taking its Fourier transform and inverting it while selectively retaining only the intensities at integer harmonic frequencies of the drive. Such a comb filtering method works particularly well in fluid TMAFM because of the highly distorted character of the deflection signal [22-24].

\subsection{Numerical model of the entire imaging process of TMAFM in solution}

To get a complete understanding of the tip/sample interaction associated with TMAFM, numeric simulations are often performed in which the cantilever is modeled as a driven damped harmonic oscillator [25], where

$$
F_{\text {ext }}=m_{\text {eff }} \ddot{z}+b \dot{z}+k\left[z-D_{o}+a_{o} \sin (\omega t)\right]
$$

$m_{\text {eff }}$ is the effective mass of a cantilever, $b$ is the damping coefficient, $k$ is the cantilever spring constant, $z$ is the position of the cantilever with respect to the surface, $D_{0}$ is the resting position of the cantilever base, $a_{0}$ is the drive oscillation amplitude, $\omega$ is the drive frequency, $t$ is the time, and $F_{\text {ext }}$ is the tip/sample force. 


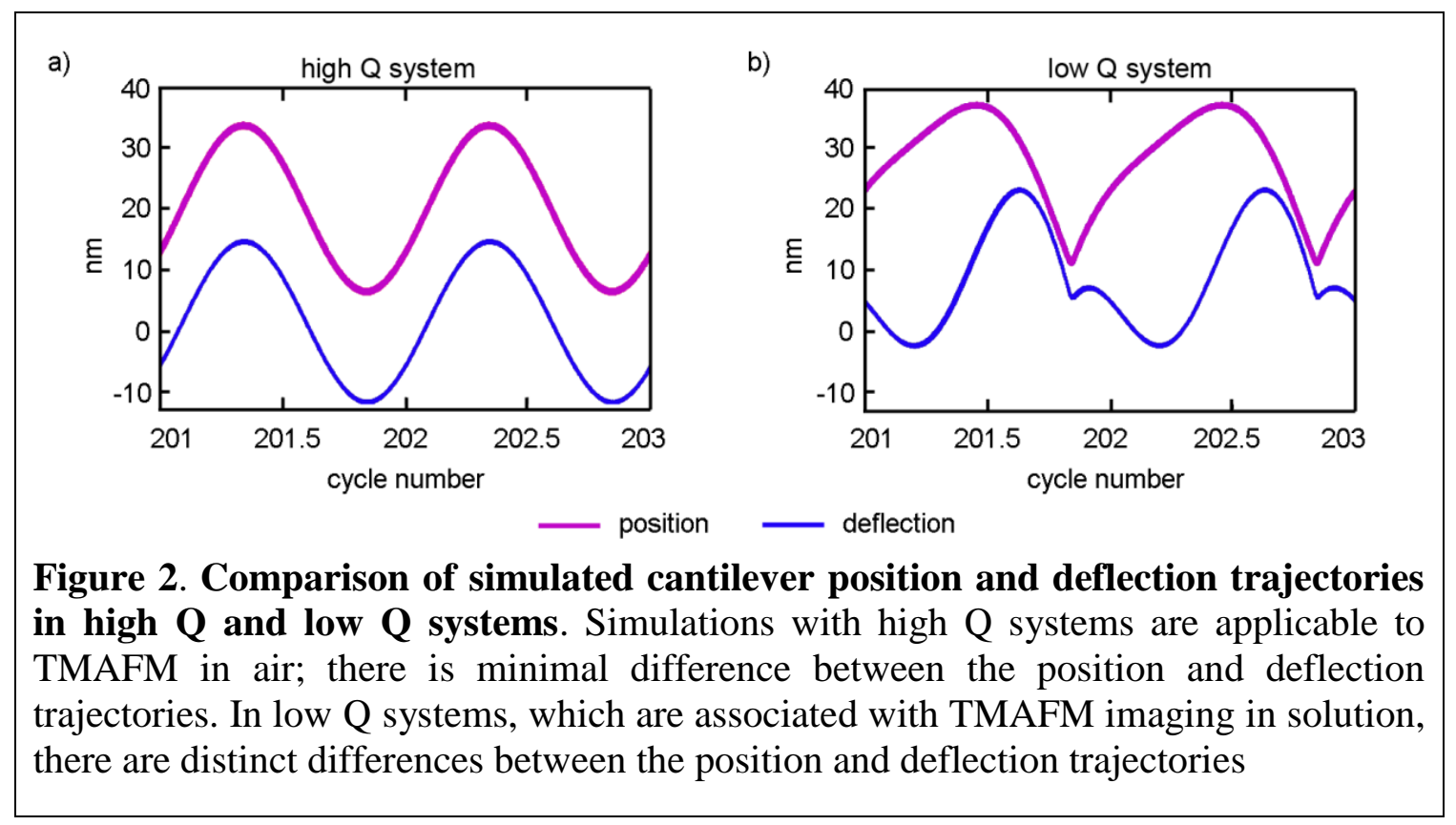

Although cantilever position, $z$, is often monitored in AFM simulations, in real experiments, most microscopes monitor the amplitude of the cantilever deflection, $y$, which is simply related to the position by:

$y=z-D_{o}+a_{o} \sin (\omega t)$

TMAFM can be operated in air or in fluids, and their major differences is in the quality factor $(Q)$ of the cantilever, $[23,26]$ described by the following relationship

$Q=A_{o} / a_{o}$

where $A_{o}$ is the free cantilever oscillation amplitude. In short, Q is the ratio of the energy stored in a system divided by the energy loss per cycle, and is typically in the range of 
$200-400$ in air, whereas in fluids it is only $\sim 1-5$. This lower Q is primarily due to the large hydrodynamic damping associated with oscillating the cantilever in fluids. In high Q systems, the free cantilever oscillation amplitude is significantly larger than the drive oscillation amplitude. When operating TMAFM in a low Q system, the cantilever deflection and position trajectories differ significantly during tapping events as the drive amplitude is comparable to the free amplitude. As a result, a characteristic anharmonic distortion is produced in the deflection trajectory as the cantilever tip contacts the sample surface (Fig. 2b). As a result, tin modeling TMAFM in solution, it is necessary to rewrite the cantilever motion in terms of deflection as

$F_{\text {ext }}=m_{\text {eff }}\left[\ddot{y}-a_{o} \omega^{2} \sin (\omega t)\right]+b\left[y+a_{o} \omega \cos (\omega t)\right]+k y$

It should be noted there are several sources of distortion in cantilever deflection signals in experimental systems, including nonlinearities of the detector and electronics of the AFM that may need to be accounted for when comparing numerical simulations to experimental results.

In TMAFM the cantilever oscillation continuously changes the separation distance between the probe tip and the surface, allowing the tip to intermittently contact the sample during each oscillation cycle. Thus, there are two tip/sample interaction regimes required for modeling the time-resolved imaging force: (a) when the cantilever tip and sample surface are not in contact and (b) when the tip and surface make contact during the tapping event. For scenario (a), the separation distance between the cantilever tip and the sample surface, $z$, is greater than the interatomic distance, $a_{D M T}$, and during the 
contact portion (b), $z$ is equal to or smaller than $a_{D M T}$. For scenario (a) the force can be described by the Derjaguin-Landau-Verway-Overbeek (DLVO) theory; [27] however, because most biological experiments are performed under near physiological conditions in a saline buffer, the force associated with the electric double layer effect is negligible, resulting in a short Debye length [28]. Thus for simplicity, the non-contact force can be approximated using the van der Waals interaction between a sphere (the cantilever tip) and a flat surface [27]

$F_{\text {ext }}=-\frac{H R_{\text {tip }}}{6 z^{2}}$ for $z>a_{D M T}$

where $H$ is the Hamaker constant and $R_{\text {tip }}$ is the tip radius [29]. For scenario (b), the tip/sample force interaction can be described using a Derjaguin-Muller-Toporov (DMT) potential,

$F_{\text {ext }}=\frac{4}{3 \kappa_{\text {eff }}} \sqrt{R_{\text {tip }}}\left(a_{D M T}-z\right)^{3 / 2}-\frac{H R_{\text {tip }}}{6 a_{D M T}^{2}}$ for $z \leq a_{D M T}$

where

$\kappa_{e f f}=\frac{1-v_{t i p}^{2}}{\pi E_{t i p}}+\frac{1-v_{\text {sample }}^{2}}{\pi E_{\text {sample }}}$

where $E$ is the Young's modulus and $v$ is the Poisson coefficient of the tip or sample.

In addition, a feedback loop equipped with an integral gain can be incorporated into this numerical model to simulate the entire TMAFM imaging process. Implementation of a feedback loop can be accomplished by systematically determining 
the cantilever amplitude for each oscillation cycle, direct comparison of this amplitude to a predetermined set-point amplitude, and adjusting the tip/surface separation distance to maintain the set-point amplitude. In this way, a simulated trace of the surface, which is simply the required correction in tip/surface separation distance, can be obtained for different model surface topographies. Combined with the ability to change the values of the Hamaker constant and/or surface Young's modulus, simulations of imaging a wide array of surfaces with TMAFM can be performed to fully understand how the timeresolved tip/sample force responds to surface properties.

\subsection{Basic principles underlying SPAM}

Equation 4 above can be rearranged to show that that the acceleration of the cantilever is directly related to the tip/sample force scaled by the effective mass of the cantilever.

$$
\ddot{y}=\frac{1}{m_{e f f}}\left[F_{e x t}-b \dot{y}-k y+m_{e f f} \omega^{2} a_{o} \sin (\omega t)-b a_{o} \omega \cos (\omega t)\right] .
$$

This suggests that by taking the second derivative of the measured deflection signal the time-resolved tip/sample force can be recovered. In its simplest form, this process amounts to treating the cantilever as a local accelerometer that can be raster scanned

across a surface. In this way, topographic and tip/sample force information can be obtained in one experiment simultaneously. This is the basis for SPAM. The last four terms of Equation 8 are all related to the action of driving the cantilever, and as a result, 
oscillate at the drive frequency. This allows these extraneous terms to be easily distinguished from the tip/sample force, or even filtered out.

As previously mentioned, real cantilever deflection trajectories contain noise. The deflection trajectory of the cantilever can be filtered using a Fourier transform based harmonic comb filter. In this process, a sliding window Fourier transform is performed on the cantilever deflection signal, only intensities in the resulting power spectrum corresponding to integer harmonic frequencies of the drive are kept, and these are used to reconstruct a deflection signal, $y_{r e c}(t)$, by an inverse Fourier transform based on the equation

$y_{\text {rec }}(t)=\hat{f}^{-1}\left[y(\omega) \sum_{k=1}^{N} \delta\left(\omega-k w_{\text {oper }}\right)\right]$

where $\delta$ is Dirac's delta function, $\omega_{\text {oper }}$ is the operating frequency, and $N$ is the highest harmonic distinguishable about the noise level. The effectiveness of the use of the comb filter to accurately reconstruct the deflection trajectory requires a sufficient number of harmonics, typically more than 18 , to be above the noise level in the power spectrum. As previously mentioned, TMAFM in fluids is characterized by a distinct distortion in the cantilever deflection trajectory associated with the tapping event, $[23,24,26]$ and this distortion inherent in fluid TMAFM results in an adequate number of higher harmonics above the noise to facilitate comb filtering and reconstruction of the tip/sample tapping force from the second derivative (or acceleration) of the cantilever deflection signal. The sliding window of this filter typically contains $5-10$ oscillation cycles, and the window is advanced one oscillation cycle at a time to maintain local information about the 
tip/sample interaction. Nonetheless, with this ability to filter cantilever deflection trajectories, spatially resolved force maps can be constructed based on the second derivative. The extraneous terms in Equation 8 can be suppressed from the reconstructed force by excluding the fundamental frequency, i.e. drive frequency, from the inverse Fourier transform; however, excluding this frequency diminishes the accuracy of the recovered force by reducing its magnitude. The reconstructed trajectory obtained from the comb filter also loses information contained in harmonics below the noise level. Furthermore, it should be noted that the harmonic comb filter should only be applied to periodic motion of the kind commonly observed in TMAFM and is not applicable to motion exhibiting period doubling or aperiodic/chaotic character. .

\subsection{Features of the time-resolved tip/sample force are independent of surface topography}

TMAFM can be used to image a wide variety of sample surfaces, with a range of topographical features. In TMAFM experiments, surface features may vary in height from sub-nanometer to several micrometers. To compare tapping mode imaging forces to mapping mechanical properties of surfaces, the features of the time-resolved tip/sample tapping force must be independent of the surface topography.

To determine the role of surface topography on tip/sample tapping forces, simulations of complete TMAFM experiments can be performed to understand the impact of a variety of model surfaces with distinct morphologies on imaging forces (Fig. 3). Here, three model surfaces are presented: 1) a region with increased RMS roughness, 2) a flat step, and 3) a 

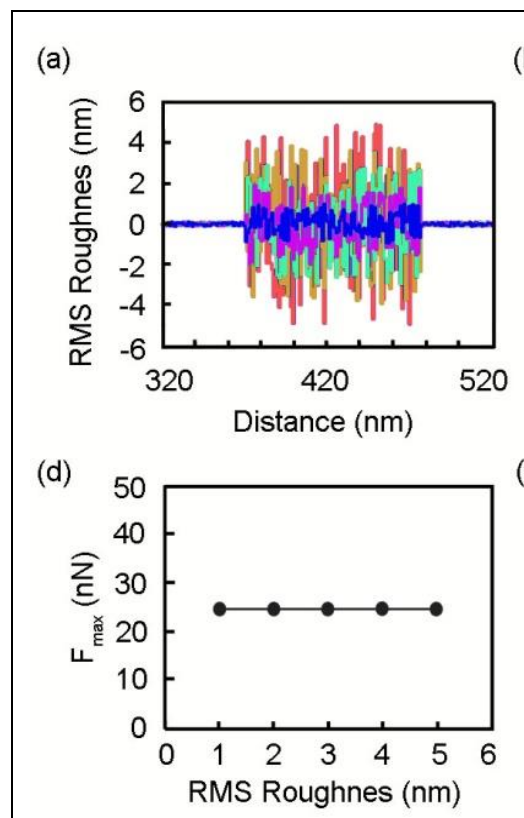

(g)

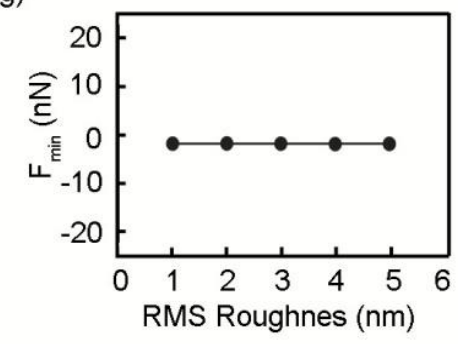

(b)

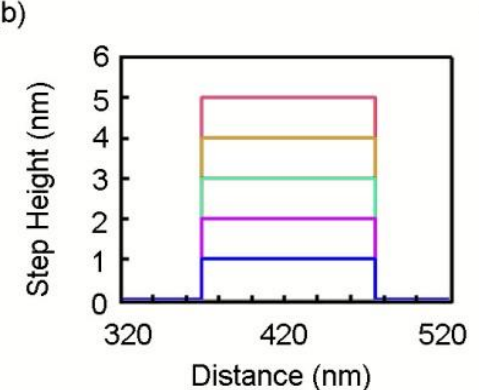

(e)

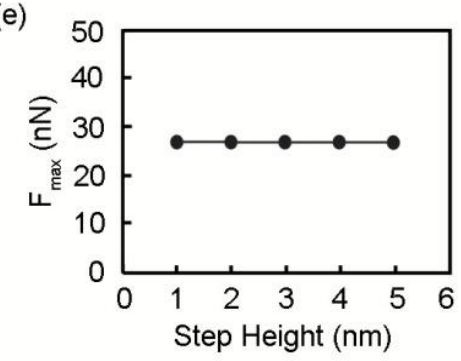

(h)

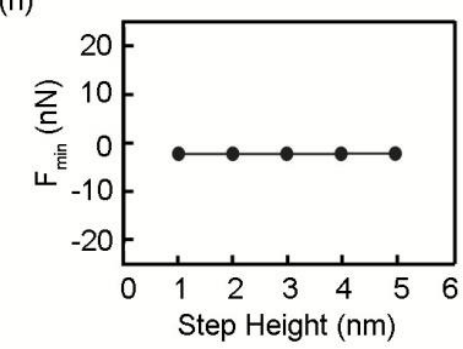

(c)

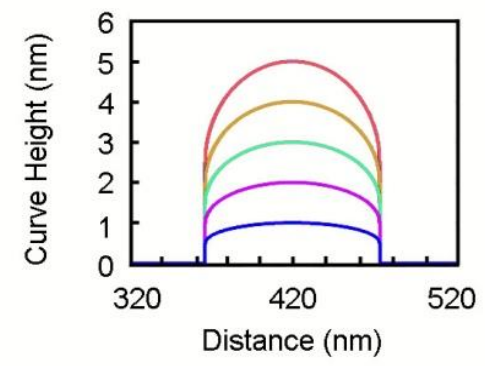

(f)

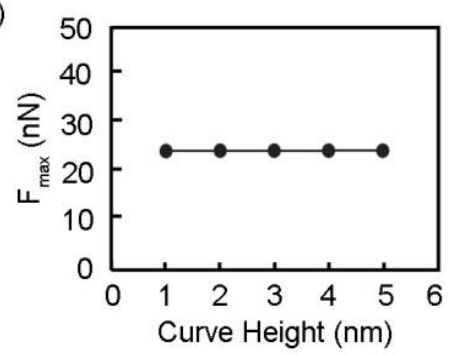

(i)

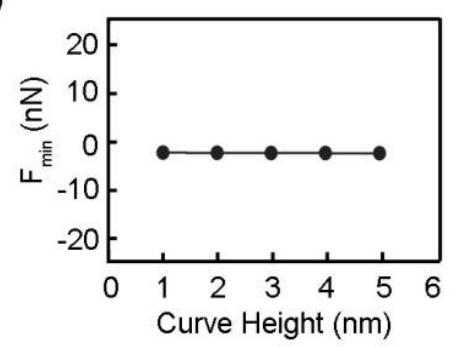

Figure 3. Simulations to determine the effect of surface topography on tip/sample imaging forces. A variety of surface topographies were modeled: (a) a rough surface, (b) a flat step, and (c) a curved feature where roughness or height was varied from 1-5 $\mathrm{nm}$. The maximum and minimum tapping forces, (d-f) and (g-i) respectively, were averaged over the altered surface topography and found to be independent of changes in surface topography.

round feature. These model surfaces represent a large number of the potential topographies that may be encountered in real AFM experiments. There are two features of the tapping force that are of interest here: (1) the maximum tapping force, $F_{\max }$, defined as the most positive force experienced between the cantilever tip and the sample surface during each cantilever oscillation cycle, and (2) the minimum tapping force, $F_{\min }$, defined as the most negative force experienced between the cantilever tip and the sample 
surface. The size of these features can be systematically increased to determine changes in $F_{\max }$ and $F_{\min }$ associated with imaging these model surfaces. All other surface characteristics (such as Young's modulus, Hamaker constant, etc.) and imaging parameters (set-point ratio, free amplitude, cantilever spring constant, etc.) were held constant in these simulations. Importantly, the average $F_{\max }$ and $F_{\min }$ associated with imaging all three of these surfaces do not change as a function of the size of the feature in the model, as long as the feedback loop is able to maintain proper tracking of the surface. $F_{\max }$ for the various topographies were averaged over the altered surface feature (the rough patch, the step, or the round feature) and had an approximate value of $25 \mathrm{nN}$ for each type of surface and each height variation (Fig. 3d-f). The $F_{\min }$ for each surface variation was approximately $-2 \mathrm{nN}$, which was exactly the same as the flat region of the surface. This indicates that as long as the feedback loop is optimized, changes in the time-resolved tapping forces are independent of topographical features.

\subsection{Application of SPAM}

Most studies utilizing SPAM have focused on supported lipid membranes as well as a variety of systems. Supported bilayers have a considerably lower elastic modulus compared commonly used surfaces such as mica and silicon, so this system provides a surface with a soft region (supported bilayer) and hard region (exposed mica). Histograms of $F_{\max }$ and $F_{\min }$ associated with imaging this system are distinctly bimodal, with each mode corresponding to either the mica or bilayer portions of surface, and providing excellent contrast in the reconstructed force images (Fig 4b-c). Consistent with the simulation results, the values of $F_{\max }$ corresponding to the bilayer were lower in 

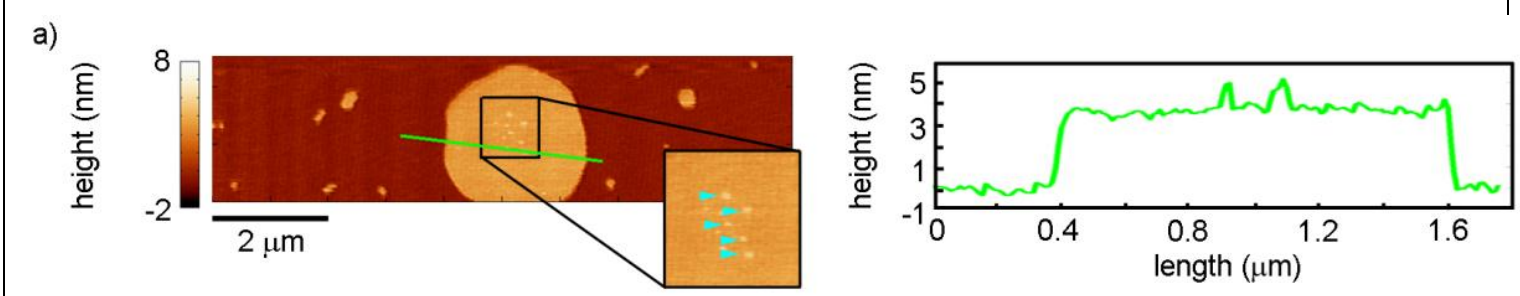

b)
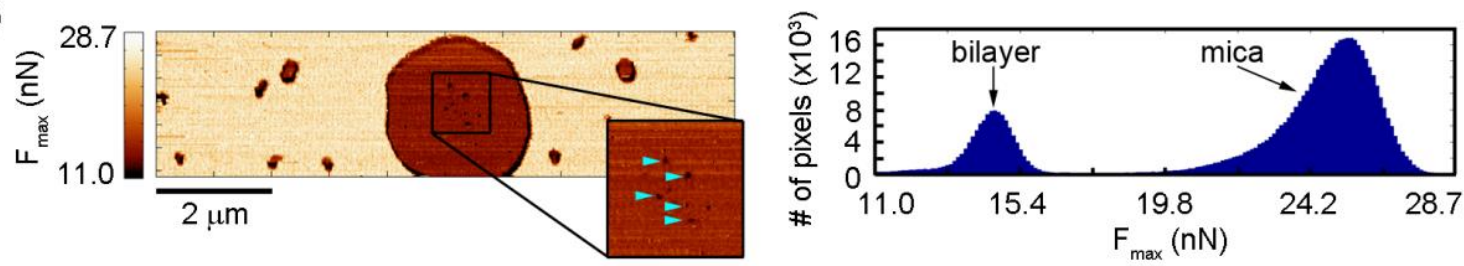

c)
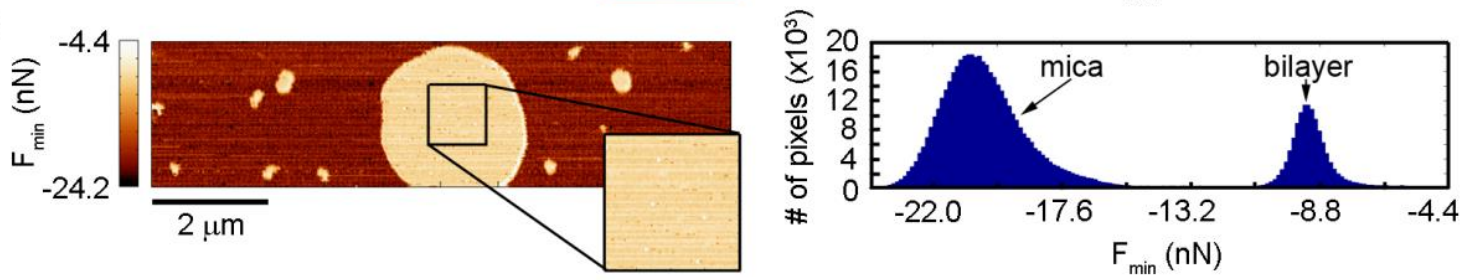

Figure 4. SPAM imaging of a supported lipid bilayer patch on mica. (a) Topography image with the green line corresponding the height profile to the left. (b) $F_{\text {max }}$ and (c) $F_{\text {min }}$ images are presented with histograms of these respective forces for every tapping event presented to the left of the images. The insets zoom in on small areas containing small, stacked bilayer on the patch (blue arrows).

comparison with those corresponding to mica. The magnitude of $F_{\min }$ associated with imaging mica was larger than that for the bilayer patch, indicating that the tip adhered more strongly to the mica surface. Several studies have measured mechanical changes in supported lipid membranes using SPAM. For example, SPAM has been used to determine the effect of cholesterol on the modulus of lipid bilayers [30], how exposure to lipoprotein particles alter membrane mechanics, [31] and how membrane disruption caused by a variety amyloid-forming proteins alter local mechanical properties of lipid bilayers [32-35]. In this chapter, SPAM was used to monitor changes in the mechanical properties during the formation of supported lipid bilayers with membrane curvature. 


\subsection{SPAM in the developed of supported lipid bilayers with curvature}

Supported lipid bilayer patches on mica substrate represented the first successful application of the SPAM technique on an experimental system. These bilayers have a considerably lower elastic modulus compared to mica, so this system provides a surface with a soft region (supported bilayer) and hard region (exposed mica). Supported lipid bilayers are commonly used as model membranes for AFM studies of protein aggregation. However, these supported bilayers usually lack curvature. By forming a bilayer on top of silica nanobeads $(50 \pm 10 \mathrm{~nm})$ deposited on a silicon substrate, model supported lipid bilayers with flat and curved regions were developed for AFM studies (Fig. 5). The presence of the bilayer over the beads was validated by continual imaging of the formation of the bilayer, height measurements, and spatially resolved mechanical measurements of the resulting bilayer using SPAM. Interpretation of this data was facilitated by numerical simulations of the entire imaging process.

\subsubsection{Preparation of total brain lipid extract}

Lyophilized total brain lipid extract (TBLE) (Avanti Polar Lipids) was dissolved in chloroform (ACROS Organics). The chloroform was evaporated using a Vacufuge plus (Eppendorf) to form a thin lipid film on the bottom walls of a micro centrifuge tube. The TBLE was reconstituted in $10 \mathrm{mM}$ Tris $\mathrm{HCl}$ buffer ( $\mathrm{pH}$ 7.3) with periodic vortexing to a concentration of $1 \mathrm{mg} / \mathrm{ml}$. To facilitate bilayer and multilayer sheet formation, the lipid sample tube went through five freeze/thaw cycles by sequential immersion in liquid nitrogen followed by thawing at $37{ }^{\circ} \mathrm{C}$ in a thermomixer (Eppendorf) [36]. The lipid sample was then bath sonicated for 45 minutes to facilitate vesicle formation. Using the 
hanging drop method, $15 \mu \mathrm{l}$ of 1:1 mixture of TBLE/Tris- $\mathrm{HCl}$ was added directly into an AFM fluid cell on a clean silicon substrate decorated with $50 \mathrm{~nm}$ silica nanoparticles (Polysciences, PA). The same lot of lipids was used in all experiments.

\subsubsection{Preparation of poly-L-lysine coated silicon substrate}

Ultra-flat silicon wafers, type $<100>$ (Ted Pella) were cut into $1 \mathrm{~cm} \mathrm{x} 1 \mathrm{~cm}$ square sizes, and sequentially sonicated in acetone, absolute ethanol, and deionized water for 20 minutes each. The wafers were then kept under water overnight and dried by blowing canned air. The clean substrates were placed under ozone for 1 hour to remove any traces of organic contaminants [37] from the acetone and ethanol. Once more, the wafers were rinsed in ultrapure water, dried with air, and placed on a hot plate at $30{ }^{\circ} \mathrm{C}$ to remove any residual solvent present. These substrates were stored in a dust free petri dish prior to use. A $25 \mu 1$ aliquot of $0.1 \%$ poly-L-lysine was evenly spread on the clean, square silicon substrate and placed on a hot plate at $30^{\circ} \mathrm{C}$ for 5 minutes, followed by rinsing with $1 \mathrm{ml}$ of deionized water to remove excess unbounded poly-L-lysine molecules [38].

\subsubsection{Nanoparticles deposition on silicon substrate}

Silica nanoparticles purchased from Polysciences were diluted to the desired concentration of $0.010 \%(0.10 \mathrm{~g} / \mathrm{L})$ with ultrapure water, followed by 1 hour of sonication prior to deposition. A $20 \mu \mathrm{l}$ drop of silica dispersion was evenly spread on the silicon substrate and allowed to slowly dry at room temperature [39]. This was rinsed with 100 $\mu 1$ of ultrapure water to remove any excess, loosely bound beads on the surface followed by air drying, and stored in a dust free environment prior to AFM experiments. 


\subsubsection{In situ AFM and SPAM imaging conditions}

A Nanoscope V Multimode AFM (Veeco,Santa Barbara,CA) equipped with a tapping mode fluid cell and an $\mathrm{O}$ ring was used to perform in situ AFM experiments. The silicon substrates with beads were scanned using a rectangular shaped silicon nitride cantilevers (Vista probes) with a nominal spring constant of $0.1 \mathrm{~N} / \mathrm{m}$. Typical scan rates ranged from 1-2 Hz, and drive frequencies of $5-6 \mathrm{kHz}$ were used. For scanning probe acceleration microscopy (SPAM) analysis,[40] topography images were captured at $512 \times 128$ pixel resolution. While the topography images were being captured, the cantilever deflection trajectories were simultaneously captured with a signal access module (Veeco) and CompuScope 14100 data acquisition card (Gage, Lachine, QC) at 2.5 MS/s and 14 bit resolution with a range of $2 \mathrm{~V}$. The captured cantilever trajectories were filtered with a sliding-window Fourier transform-based harmonic comb filter, and the second derivatives of the filtered cantilever deflection trajectories were taken and multiplied by the effective mass, $m_{\mathrm{eff}}$, of the cantilever to obtain the time-resolved-based tapping force between the tip and sample. To understand the tip/sample forces, we carried out numerical simulations in which the cantilever was modeled as a driven damped harmonic oscillator as previously described. 


\subsection{Results}

\subsubsection{Development of a system containing supported curved and flat lipid bilayers.}

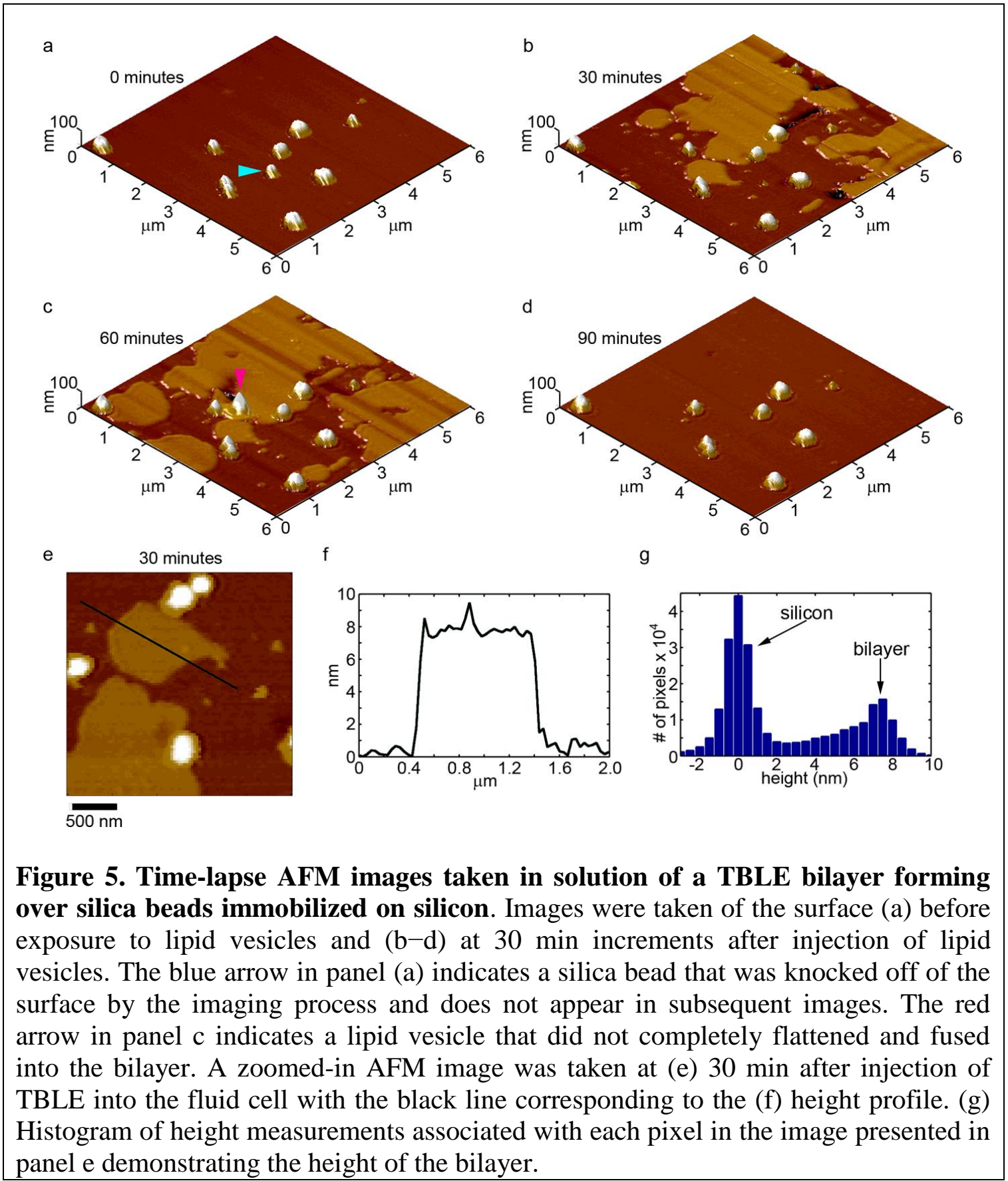


To provide a substrate capable of inducing well-defined, local regions of curvature in a supported bilayer, the strategy of using silica beads (diameter of $50 \pm 10$ $\mathrm{nm})$ that were immobilized on clean silicon surfaces pre-treated with $0.1 \%$ poly-L-lysine as substrates was employed. These substrates were subsequently exposed to vesicles comprised of TBLE, resulting in the formation of a lipid bilayer on the surface via the process of vesicles flattening and fusing on the surface (Fig. 5b-c). This strategy would provide both curved and flat bilayers in the same system, and would allow for the direct tracking of htt aggregation on these two distinct regions by AFM imaging in solution. While the beads were found to be firmly attached to the surface, the process of acquiring AFM images and/or injecting solutions containing vesicle into the fluid cell did result in rare instances where a bead was displaced from the surface (Fig. 5a, blue arrow). The formation of the lipid bilayer on the flat portions of the substrate could be tracked by continuous AFM imaging of the scan area, and smooth bilayer completely covered these flat regions of the surface typically within $\sim 90$ minutes. At intermediate time points, the thickness of the bilayer could be determined from analysis of AFM height images. The supported bilayer above the flat silicon substrate was $7.3 \pm 0.4 \mathrm{~nm}$, as demonstrated by height profiles and histograms of the height associated with every pixel in the image (Fig. $5 e-g)$.

Despite the obvious formation of lipid bilayer on the flat portions of the substrate, there are two primary ways the bilayer can form to accommodate the presence of the immobilized bead: 1) by forming a pore around the periphery of the bead or 2) by forming a bilayer over the top of the bead, thus enveloping it (Fig. 6). Both scenarios have been observed in the literature [39]. The first scenario is not useful for this study, as 


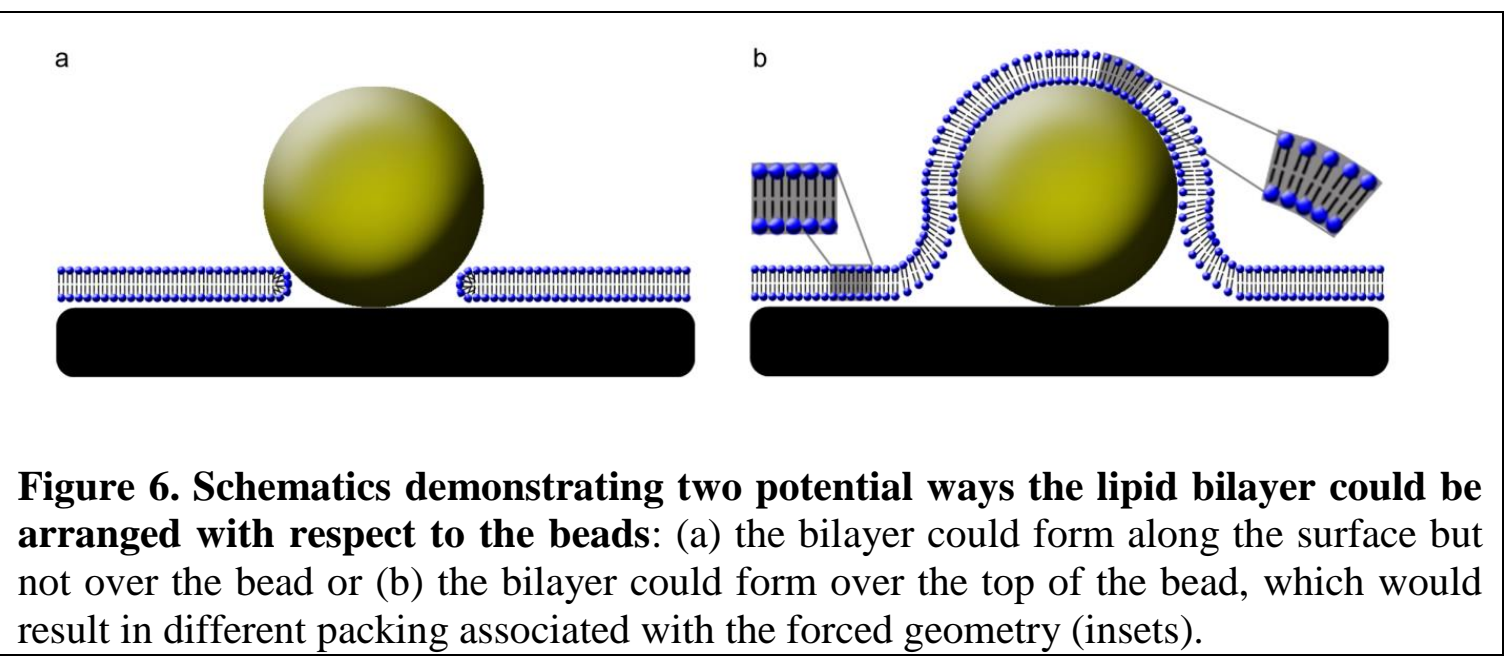

it would not result in a curved model bilayer; however, the second scenario would provide such a surface. As a result, the formation of the bilayer forming over the top of the beads, thus encapsulating them, needed to be verified. Previous studies used comparison of the height of the bead and phase imaging before and after addition of lipid to determine of the bilayer formed over the bead [39]. The logic behind this approach is that if the bilayer forms on the flat substrate and on top of the bead that there would be no apparent change in height of the bead. If the bilayer formed a pore around the bead, than the observed bead height would be decreased by the thickness of the bilayer. Therefore, the average height of the exact same beads immobilized on the silicon surface were compared before addition of any lipid and after the bilayer appeared to have completely covered the flat portions of the substrate. There was an observed decrease of $2.9 \mathrm{~nm}$ in the average bead height associated with the presence of the lipid bilayer. As the bilayer on the flat region was known to have an average height of $7.3 \mathrm{~nm}$, this smaller observed height suggests that some form of lipid did indeed deposit over the top of the beads, but that there is also something physically different between the lipid on top of the bead and the flat portion of the substrate. 


\subsubsection{Validating bilayer formation on beads using imaging forces.}

Analysis of the time-resolved imaging forces associated with tapping mode AFM was performed in order to provide an understanding of why this small height different was observed and providing further validation that bilayer had indeed formed over the top of the bead. These imaging forces have been demonstrated to be highly dependent on surface mechanical properties, and in particular, the maximum tapping force $\left(F_{\max }\right)$, defined as the peak or largest force occurring between the tip and surface during a single tapping event, can be used as an indirect measure of the compression or Young's modulus of a sample. While AFM topography images were obtained of the beads on silicon before and after the formation of a lipid bilayer, the entire cantilever deflection signal was captured and processed to recover the time-resolved tip/sample tapping force by SPAM. In this way, the tapping forces can be associated with specific features in the topography image and used to make surface maps of mechanical properties. To aid in interpreting these force maps and directly relating them to the observed height measurements of the beads, several numerical simulations of the complete tapping mode AFM imaging process in solution were performed $[33,36,41]$.

The rigidity of a surface can be modeled by varying the Young's modulus of the sample in equation 7. A series of simulations were run with Young's modulus values of the model surface ranging from 0.5 to $100 \mathrm{GPa}$ (Fig. 7). All other imaging parameters were held constant, e.g., cantilever free amplitude, spring constant, and set-point ratio, when imaging these model surfaces. As a result, the total tip/sample imaging force over an entire cantilever oscillation cycle was constant; however, this force was distributed 
a

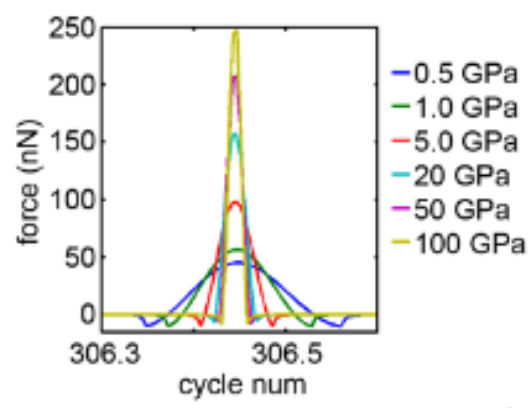

b

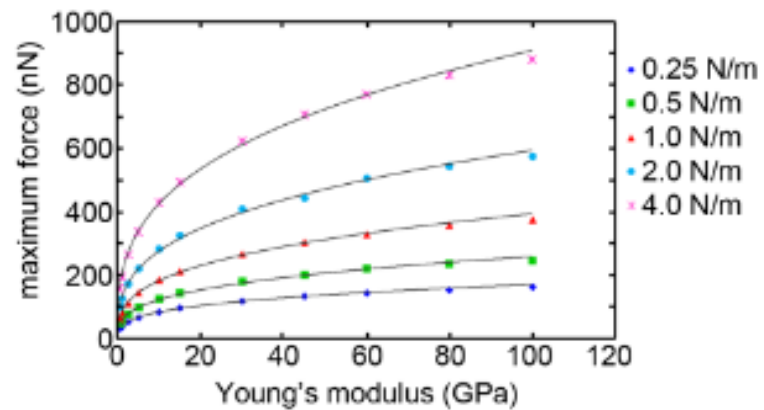

Figure 7. Tapping mode AFM simulations demonstrate the relationship between imaging forces and surface mechanical properties. (a) Simulated time-resolved tip/sample forces associated with tapping mode AFM imaging on surfaces with various values of Young's modulus. (b) Maximum tapping force as a function of the surface Young's modulus for cantilevers with different spring constants.

differently over the entire tapping event (Fig. 7a). As the surface became increasingly more compliant, the contact time between the tip and surface increases, resulting in the imaging force being distributed over this longer contact time with a corresponding decrease in the $F_{\max }$ associated with the tapping event. This decrease in $F_{\max }$ with smaller values of surface Young's modulus followed a power law dependence (Fig. 7b). For any given set of imaging parameters, this power law dependence between $F_{\max }$ and the surface Young's modulus was observed; although, the magnitude of the forces would vary. For example, increasing the cantilever spring constant resulted in larger values of $F_{\max }$, but for any given spring constant, the power law dependence between $F_{\max }$ and Young's modulus was observed (Fig. 7b). This demonstrates that for any given set of imaging parameters that $F_{\max }$ can be used as a way to compare the relative rigidity of features within an AFM image. 


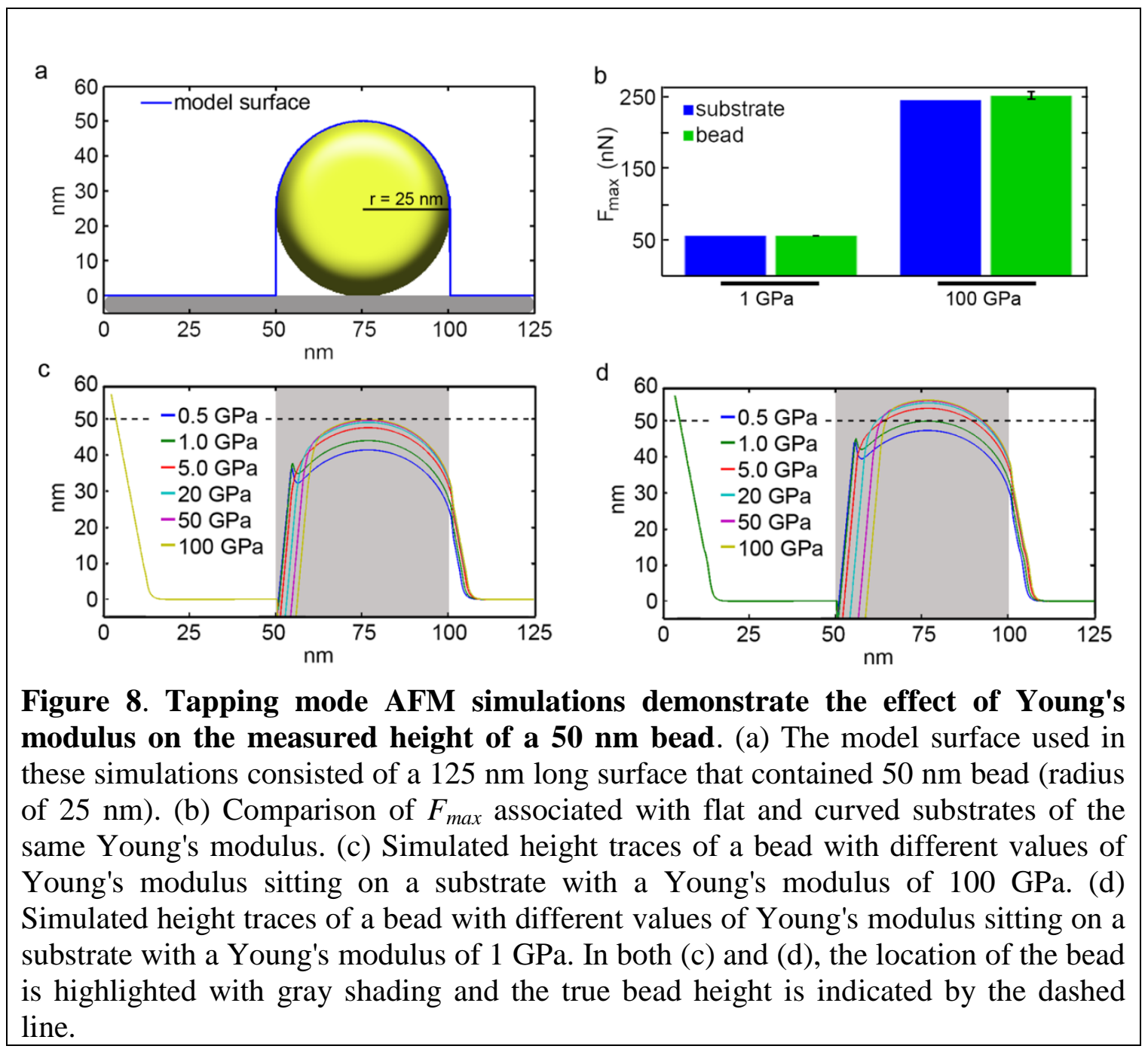

Next, numerical simulations of imaging a bead deposited on a surface via tapping mode AFM in solution were performed. The model surface used in these simulations was $125 \mathrm{~nm}$ long, and contained a $50 \mathrm{~nm}$ wide curved region that was $50 \mathrm{~nm}$ in height and flanked by flat regions (Fig. 8a). The Young's modulus of the flat and curved regions of the model surface could be independently altered. First, simulations were performed to determine if changes in $F_{\max }$ could potentially be associated with the curvature associated with the beads. When the Young's modulus of the flat and curved regions of the model surface was equal, there was no observed change in $F_{\max }$ (Fig. 8b). When the Young's 


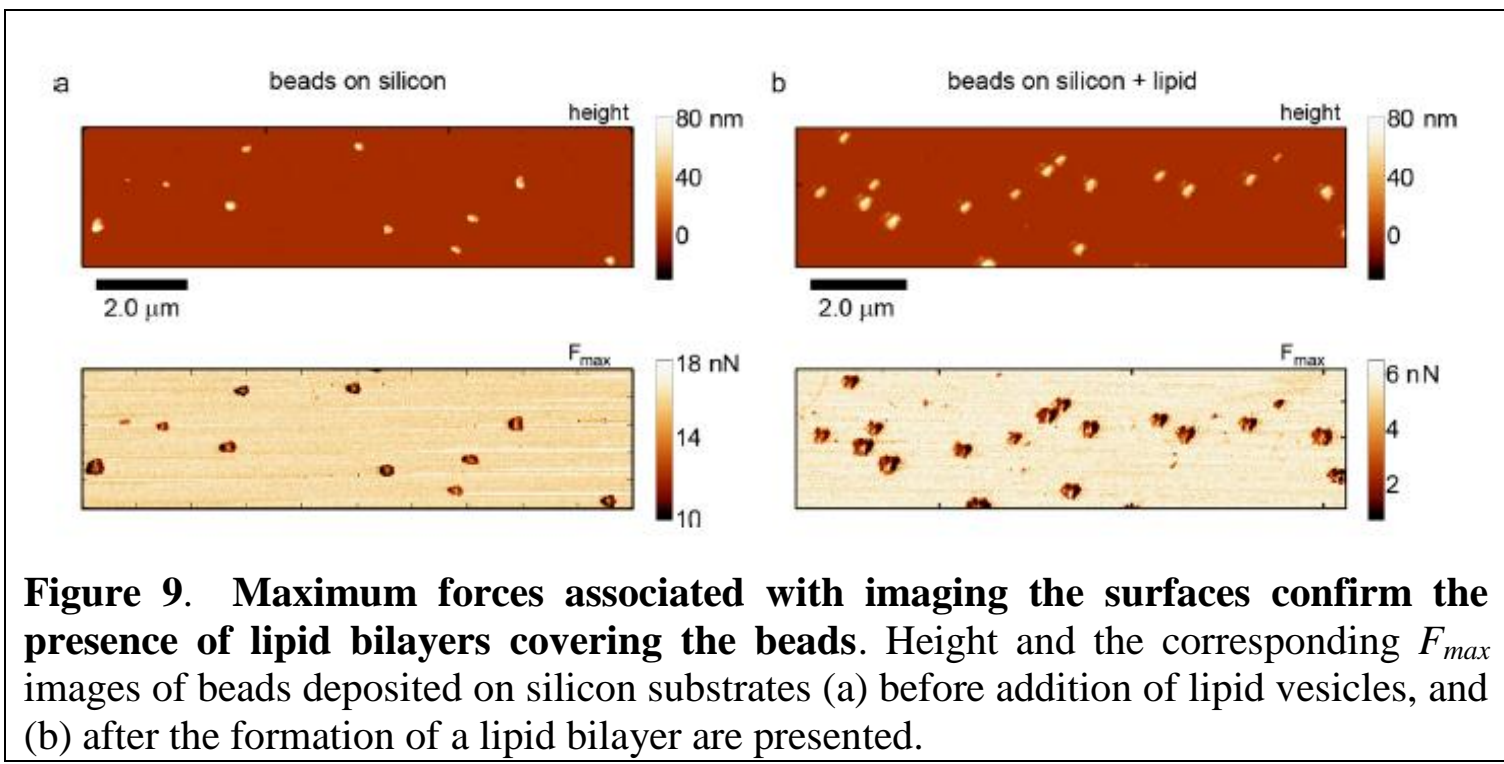

modulus differed between the flat and curved regions, $F_{\max }$ changed as would be predicted based on the power law dependence, that is, the softer region was always associated with a smaller $F_{\max }$ regardless if this corresponded to the flat or curved region of the surface. Next, the measured height of the model bead with varying values of Young's modulus (ranging from $0.5 \mathrm{GPa}$ to $100 \mathrm{GPa}$ ) with respect to the flat region of the surface, which had a Young's modulus of $100 \mathrm{GPa}$ (Fig. 8c) or $1.0 \mathrm{GPa}$ (Fig. 8d), was determined. When the bead and flat regions of the model surface had the same Young's modulus, the measured height of the bead was the same as the true height of the bead, i.e. $50 \mathrm{~nm}$. Whenever the flat region of the model surface was more rigid compared to the bead, the observed height was smaller compared to the true height of the bead with the observed height becoming increasingly smaller with lower values of Young's modulus. If the flat portion of the surface was softer than the bead, the observed height of the bead became systematically larger than the true height as the Young's modulus of the bead 
increased. The deviation of the height of the bead from the true height is a result of the applied force associated with the imaging process compressing the softer regions of the surface to a greater extent, which forms the basis of compliance based contrast in AFM. Keeping the results of the AFM simulations in mind and to confirm the presence of a lipid bilayer on top of the beads, the relative local rigidity of actual surfaces was mapped before and after the formation of the lipid bilayer. This was accomplished by reconstructing the time-resolved tip/sample force associated with every tapping event while obtaining AFM images by SPAM, allowing $F_{\max }$ (and thus rigidity) to be correlated to specific regions of the surface. There was stark contrast in the $F_{\max }$ image between beads and bare silicon (Fig. 9). The magnitude of the force indicated that the beads were softer compared to the bare substrate, which is consistent with the literature values of Young's modulus for the two materials. The bulk Young's modulus of silica is $\sim 73 \mathrm{GPa}$, and the Young's modulus of silicon is typically >100 GPa. After a bilayer was observed to completely form on the flat portion of the substrate, the magnitude of $F_{\max }$ corresponding to the location of the beads was smaller than the surrounding bilayer. As supported lipid bilayers have a Young's modulus on the order of $1 \mathrm{GPa}$ or smaller, the $F_{\max }$ associated with an exposed silica bead would be larger. Thus, observed $F_{\max }$ suggests that a lipid bilayer covered the beads and that the curved bilayers on top of beads were more compliant (smaller Young's modulus) than the flat supported bilayers. Based on the simulations, the increased compliance would result in a smaller measured height of the bead as the tip compresses the curved bilayer to a larger extent compared to the flat bilayer. The reduced Young's modulus of bilayers on top of the beads was most 
likely due to altered packing of the lipid components due to the induced curvature (Fig. $6 b$ insets).

\subsection{Discussion}

While tapping mode AFM in solution is widely used to study morphological and mechanical properties of supported lipid bilayers, one of the limitations associated with comparing these results to physiologically relevant membranes is the lack of membrane curvature associated with such systems. A potential method to induce curvature in supported lipid membranes is introducing local curvature in the substrate. This was accomplished by depositing silica nanobeads onto a silicon substrate, and by the process of vesicle fusion, formed a supported lipid bilayer that covered the flat silicon substrate and curved beads. Verification of the lipid bilayer forming on top of the beads was provided by a combination of height measurements and analysis of time-resolved tip/sample forces. Interpretation of both height and force analysis was aided by numerical simulations of the entire imaging process involving a model surface of the correct topography. Interestingly, $F_{\max }$ revealed that not only did lipid organize on top of the beads, but that bilayers on top of the beads had a qualitative reduction in the Young's modulus compared to bilayer supported by flat regions of the substrate, which was consistent with alterations in lipid packing associated with induced curvature [42, 43]. Collectively, these results demonstrate that membrane curvature can significantly alter bilayer mechanical properties and that these changes can be detected by AFM tip/sample force reconstruction. 
With the validation that supported lipid bilayers formed over the beads, a model system that allowed studies to simultaneously monitor the interaction of proteins with curved or flat regions of a bilayer was developed. There are several advantages associated with such an approach. Mechanical measurements confirmed that the bilayer on top of the beads was mechanically, and thus structurally, different compared with the flat portions of the bilayer, and this is one of the advantages of using AFM for these types of studies. Though, TBLE was used as the lipid system, this approach should be amenable to various lipid systems as well. Due to the uniform size of the beads, the curved portions of the supported bilayer are relatively uniform. In theory, the curvature can be modified by changing the size of the beads; however, encapsulation of immobilized beads by a lipid bilayer does not occur on all bead sizes, as has been previously observed [39, 44]. This reinforces the importance of confirming the formation of the bilayer over the top of the beads, and lack of such confirmation is the main reason beads of varying size were not used for the studies presented here. Another caveat associated with this approach that should be noted is that these bilayers are still supported, and underlying substrates may influence the fluidity and other properties of lipid membranes.

\subsection{Conclusions}

In conclusion, supported lipid bilayers with curvature may fight useful applications, and such can be exposed to amyloid-forming proteins to determine the impact of membrane curvature on protein/lipid interactions associated with diseases. It can also provide indispensable information on nanobeads-lipid membrane interaction, and cytotoxicity. 


\subsection{References}

1. Zhong, Q., et al., Fractured polymer/silica fiber surface studied by tapping mode atomic force microscopy. Surface Science, 1993. 290(1-2): p. L688-L692.

2. Burnham, N.A., et al., How does a tip tap? Nanotechnology, 1997. 8(2): p. 67-75.

3. Nony, L., R. Boisgard, and J.P. Aime, Nonlinear dynamical properties of an oscillating tip-cantilever system in the tapping mode. Journal of Chemical Physics, 1999. 111(4): p. 1615-1627.

4. Cleveland, J.P., et al., Energy dissipation in tapping-mode atomic force microscopy. Applied Physics Letters, 1998. 72(20): p. 2613-2615.

5. Tamayo, J. and R. Garcia, Relationship between phase shift and energy dissipation in tapping-mode scanning force microscopy. Applied Physics Letters, 1998. 73(20): p. 2926-2928.

6. Zitzler, L., S. Herminghaus, and F. Mugele, Capillary forces in tapping mode atomic force microscopy. Physical Review B, 2002. 66(15): p. 155436.

7. Garcia, R., J. Tamayo, and A. San Paulo, Phase contrast and surface energy hysteresis in tapping mode scanning force microsopy. Surface and Interface Analysis, 1999. 27(5-6): p. 312-316.

8. Stark, M., et al., From Images to Interactions: High-Resolution Phase Imaging in Tapping-Mode Atomic Force Microscopy. Biophysical Journal, 2001. 80(6): p. 3009-3018. 
9. Marcus, M.S., et al., Material anisotropy revealed by phase contrast in intermittent contact atomic force microscopy. Physical Review Letters, 2002. 88(22): p.226103

10. Melcher, J., et al., Origins of phase contrast in the atomic force microscope in liquids. Proceedings of the National Academy of Sciences of the United States of America, 2009. 106(33): p. 13655-13660.

11. Stark, M., et al., Inverting dynamic force microscopy: From signals to timeresolved interaction forces. Proceedings of the National Academy of Sciences of the United States of America, 2002. 99(13): p. 8473-8478.

12. Balantekin, M., A.G. Onaran, and F.L. Degertekin, Quantitative mechanical characterization of materials at the nanoscale through direct measurement of time-resolved tip-sample interaction forces. Nanotechnology, 2008. 19(8).

13. Garcia, R. and E.T. Herruzo, The emergence of multifrequency force microscopy. Nature Nanotechnology, 2012. 7(4): p. 217-226.

14. Legleiter, J., et al., Scanning probe acceleration microscopy (SPAM) in fluids: mapping mechanical properties of surfaces at the nanoscale. Proc. Natl. Acad. Sci. U. S. A., 2006. 103: p. 4813-4818.

15. Sahin, O., et al., An atomic force microscope tip designed to measure timevarying nanomechanical forces. Nature Nanotechnology, 2007. 2(8): p. 507-514.

16. Sahin, O., et al., Resonant harmonic response in tapping-mode atomic force microscopy. Physical Review B, 2004. 69(16).

17. Sahin, O., et al., High-resolution imaging of elastic properties using harmonic cantilevers. Sensors and Actuators a-Physical, 2004. 114(2-3): p. 183-190. 
18. Sarioglu, A.F., S. Magonov, and O. Solgaard, Tapping-mode force spectroscopy using cantilevers with interferometric high-bandwidth force sensors. Applied Physics Letters, 2012. 100(5).

19. Sarioglu, A.F. and O. Solgaard, Modeling, design, and analysis of interferometric cantilevers for time-resolved force measurements in tapping-mode atomic force microscopy. Journal of Applied Physics, 2011. 109(6).

20. Hillenbrand, R., M. Stark, and R. Guckenberger, Higher-harmonics generation in tapping-mode atomic-force microscopy: Insights into the tip-sample interaction. Applied Physics Letters, 2000. 76(23): p. 3478-3480.

21. Stark, R.W. and W.M. Hecki, Higher harmonics imaging in tapping-mode atomic-force microscopy. Review of Scientific Instruments, 2003. 74(12): p. 5111.

22. Chen, G.Y., et al., Transient response of tapping scanning force microscopy in liquids. Journal of Vacuum Science \& Technology B, 1996. 14(2): p. 1313-1317.

23. Legleiter, J. and T. Kowalewski, Insights into fluid tapping-mode atomic force microscopy provided by numerical simulations. Applied Physics Letters, 2005. 87(16): p. 163120

24. Putman, C.A.J., et al., Tapping mode atomic-force microscopy in liquid. Applied Physics Letters, 1994. 64(18): p. 2454-2456.

25. Garcia, R. and R. Perez, Dynamic atomic force microscopy methods. Surface Science Reports, 2002. 47(6-8): p. 197-301.

26. Tamayo, J., Energy dissipation in tapping-mode scanning force microscopy with low quality factors. Applied Physics Letters, 1999. 75(22): p. 3569-3571. 
27. Israelachvili, J., Intermolecular \& Surface Forces, 1992, London: Academic Press.

28. Xu, X., et al., Unmasking imaging forces on soft biological samples in liquids when using dynamic atomic force microscopy: A case study on viral capsids. Biophysical Journal, 2008. 95(5): p. 2520-2528.

29. Derjaguin, B.V., V.M. Muller, and Y.P. Toporov, Effect of contact deformations on the adhesion of particles. Journal of Colloid and Interface Science, 1975. 53(2): p. 314-326.

30. Shamitko-Klingensmith, N., et al., Mapping the Mechanical Properties of Cholesterol-Containing Supported Lipid Bilayers with Nanoscale Spatial Resolution. Langmuir, 2012. 28: p. 13411-13422.

31. Legleiter, J., et al., The Modulating Effect of Mechanical Changes in Lipid Bilayers Caused by ApoE-Containing Lipoproteins on A $\beta$ Induced Membrane Disruption. ACS Chem. Neuroscience., 2011. 2(10): p. 588-599.

32. Yates, E.A., et al., Specific domains of abeta facilitate aggregation on and association with lipid bilayers. Journal of molecular biology, 2013. 425(11): p. 1915-33.

33. Burke, K.A., E.A. Yates, and J. Legleiter, Amyloid-Forming Proteins Alter the Local Mechanical Properties of Lipid Membranes. Biochemistry, 2013. 52(5): p. 808-817.

34. Burke, K.A., et al., The interaction of polyglutamine peptides with lipid membranes is regulated by flanking sequences associated with huntingtin. The Journal of biological chemistry, 2013. 288(21): p. 14993-5005. 
35. Burke, K.A., et al., Huntingtin disrupts lipid bilayers in a polyQ-length dependent manner. Biochimica Et Biophysica Acta, 2013. 1828(8): p. 1953-61.

36. Shamitko-Klingensmith, N., et al., Mapping the mechanical properties of cholesterol-containing supported lipid bilayers with nanoscale spatial resolution. Langmuir : the ACS journal of surfaces and colloids, 2012. 28(37): p. 13411-22.

37. Sundh, M., S. Svedhem, and D.S. Sutherland, Formation of Supported Lipid Bilayers at Surfaces with Controlled Curvatures: Influence of Lipid Charge. The Journal of Physical Chemistry B, 2011. 115(24): p. 7838-7848.

38. Zhang, W., J. Hughes, and Y. Chen, Impacts of Hematite Nanoparticle Exposure on Biomechanical, Adhesive, and Surface Electrical Properties of Escherichia coli Cells. Applied and Environmental Microbiology, 2012. 78(11): p. 3905-3915.

39. Roiter, Y., et al., Interaction of Nanoparticles with Lipid Membrane. Nano Letters, 2008. 8(3): p. 941-944.

40. Legleiter, J., et al., Scanning probe acceleration microscopy (SPAM) in fluids: Mapping mechanical properties of surfaces at the nanoscale. Proceedings of the National Academy of Sciences of the United States of America, 2006. 103(13): p. 4813-4818.

41. Legleiter, J. and T. Kowalewski, Insights into fluid tapping-mode atomic force microscopy provided by numerical simulations. Applied Physics Letters, 2005. 87(16): p. 163120

42. Vamparys, L., et al., Conical Lipids in Flat Bilayers Induce Packing Defects Similar to that Induced by Positive Curvature. Biophysical Journal, 2013. 104(3): p. 585-593. 
43. Vanni, S., et al., Amphipathic Lipid Packing Sensor Motifs: Probing Bilayer Defects with Hydrophobic Residues. Biophysical Journal, 2013. 104(3): p. 575584.

44. Roiter, Y., et al., Interaction of Lipid Membrane with Nanostructured Surfaces. Langmuir, 2009. 25(11): p. 6287-6299. 


\section{Curvature enhances binding and aggregation of huntingtin at lipid membranes ${ }^{1}$}

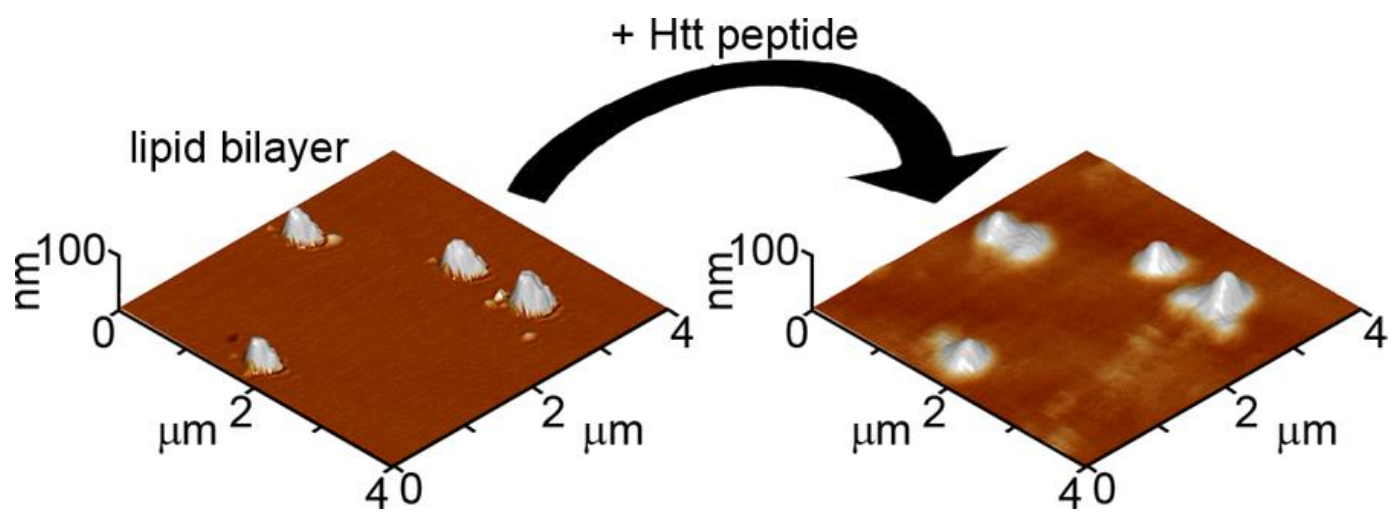

Huntington disease (HD) is a genetic, autosomal neurodegenerative disease caused by an expanded polyglutamine (polyQ) in the first exon of the huntingtin (htt) protein, facilitating its aggregation. Htt interacts with a variety of membranous structures within the cell, and the first seventeen amino acids $\left(\mathrm{Nt}^{17}\right)$ of htt directly flanking the polyQ domain is an amphiphathic $\alpha$-helix (AH) lipid-binding domain. AHs are also known to detect membrane curvature. To determine if htt exon1 preferentially bound curved membranes, in situ atomic force microscopy (AFM) studies were performed. Supported lipid bilayers are commonly used as model membranes for AFM studies of protein aggregation. However, these supported bilayers usually lack curvature. Supported lipid bilayers with curvature were developed as described in chapter 2. This model bilayer system was exposed to synthetic truncated htt exon1 peptide $\left(\mathrm{Nt}^{17} \mathrm{Q}_{35} \mathrm{P}_{10} \mathrm{KK}\right)$, and this peptide preferentially bound and accumulated to curved membranes, consistent with the ability of AHs to sense membrane curvature.

\footnotetext{
${ }^{1}$ This chapter is based on "Curvature enhances binding and aggregation of huntingtin at lipid membranes"
} 


\subsection{Introduction: Huntingtin's disease and Importance of membrane curvature}

Huntington's disease, a fatal neurodegenerative disease, is caused by an expansion of a polyglutamine (polyQ) domain in the first exon of the htt [1]. Lying near the $\mathrm{N}$ terminus of the protein, expansion of polyQ beyond a critical threshold ( $\sim 35-39$ repeat residues) directly leads to the formation of a variety of htt aggregates, i.e. oligomers and fibrils, with the eventual formation of inclusion bodies in HD brain tissues [2]. The age of onset and severity of disease are strongly correlated with the length of the polyQ expansion [3]. Full length htt is over 3144 amino acids in size; however, a large number of truncated htt products are produced in vivo via proteolysis,[4-6] and several lines of evidence suggest that the first exon of htt may play an important role in HD. For example, the expression of htt exon1 with an expanded polyQ tract causes a progressive neurological phenotype in transgenic mice [2,7], and N-terminal fragments comparable to exon1 are detected in knock-in mouse models of HD that express full-length htt $[2,7]$ and in HD patients [5].

Structural analysis of the first 17 amino acids of htt exon1 $\left(\mathrm{Nt}^{17}\right)$ directly preceding the polyQ tract suggests it functions as a lipid binding domain as it forms an amphiphilic $\alpha$-helix (AH) [8-10]. The addition of $\mathrm{Nt}^{17}$ shifts the aggregation process of polyQ in free solution to a pathway that includes oligomeric intermediates [11-13]. Further studies suggest that surfaces, including lipid membranes, can further modulate the aggregation process, favoring the formation of amorphous accumulations of htt exon1 and small oligomers $[14,15]$. Targeting the $\mathrm{Nt}^{17}$ domain with other molecules is an effective way of altering htt aggregation [11, 16]. A polyproline (polyP) domain is 
directly adjacent to the C-terminal side of the polyQ domain in htt. The polyP sequence also influences the aggregation kinetic, conformational, and structural properties of htt $[17,18]$. The aggregation of htt on model lipid membranes is also modulated by the presence of the polyP domain [15].

Upon subcellular fractionation of neuron-like clonal striatal cells, 50\% of endogenous htt partitions with membranes [19]. Association of htt with lipids is further supported by the accumulation of lipid species in htt aggregates in transgenic mouse models of $\mathrm{HD}[20,21]$. Beyond playing a role in targeting lipid membranes, $\mathrm{Nt}^{17}$ functions in trafficking htt to the endoplasmic reticulum, mitochondria, Golgi, and various vesicles [22-24]. N-terminal fragments of htt are observed in membranes that have been isolated from brain [4]. An apparent correlation of polyQ length with insertion into lipid membranes, $[14,25]$ suggests that htt's interactions with lipids may play a role in HD. In this regard, htt has been shown to disrupt bilayer structure, altering the local mechanical properties of supported membranes $[14,26]$.

A variety of protein motifs play a critical role in sensing and targeting specific physical properties of membranes. In particular, membrane curvature is a physical property targeted by amphipathic helices $(\mathrm{AH})$ [27-29]. As the $\mathrm{Nt}^{17}$ domain of htt has been shown to have an amphipathic helical structure as a monomer, [30] fibril, [12] and when bound to lipids, $[9,10,12]$ this study sought to determine if htt preferentially binds to curved membranes. In recent years, supported lipid bilayers have been increasingly used as model substrates for AFM studies aimed at understanding protein aggregation on membranes; [26, 31-36] however, as these supported bilayers are typically flat, it is 
difficult to account for the potential role of membrane curvature in protein/lipid interaction using this method. In chapter 2, a system of a model supported lipid bilayers with both flat and curved regions were developed and validated. This system is exposed to a model htt peptide to determine if curvature plays a role in the interaction of htt with lipid membranes. The model, synthetic htt peptide was chosen based on several considerations: 1) 35 Gln repeats in synthetic peptides is sufficient for aggregation;[13] 2) the polyP domain length (10 prolines) is similar to that directly adjacent to polyQ in htt exon1; 3) the known lipid-binding properties of $\mathrm{Nt}^{17}$;[22] and 4) this peptide has been demonstrated to effect lipid membranes in a similar manner to full htt exon1 proteins [15].

\subsection{Materials and Methods}

\subsubsection{In situ AFM imaging conditions}

In situ AFM experiments were performed with a Nanoscope V MultiMode scanning probe microscope (Veeco, Santa Barbara, CA) equipped with a with a tapping mode fluid cell, an $\mathrm{O}$ ring, and a closed-loop vertical engage J-scanner. Images were taken with rectangular shaped silicon nitride cantilevers (Vista probes) with a nominal spring constant of $0.1 \mathrm{~N} / \mathrm{m}$ (Phoenix, AZ). Typical scan rates ranged from 1-2 Hz, and drive frequencies of $5-6 \mathrm{kHz}$ were used.

\subsubsection{Peptide Preparation}

A synthetic peptide based on Htt exon1, Nt17 $\mathrm{Q}_{35} \mathrm{P}_{10} \mathrm{KK}$, was obtained via custom synthesis (Keck Biotechnology Resource Laboratory, New Haven, CT). Disaggregation 
and solubilization of the peptides was achieved based on established protocols.[37] Briefly, crude peptide was dissolved for $3 \mathrm{~h}$ in a 1:1 mixture of trifluoroacetic acid (Acros Organics) and hexafluoroisopropanol (Acros Organics) at a concentration of $0.5 \mathrm{mg} / \mathrm{ml}$. Samples were rigorously vortexed, and the solvent was then evaporated off with a gentle stream of $\mathrm{N}_{2}$. To remove any trace amounts of solvent from the peptide, samples were placed in a Vacufuge concentrator (Eppendorf). This process produced thin peptide films that were resuspended in ultrapure water adjusted to $\mathrm{pH} 3$ with trifluoroacetic acid to 200 $\mu \mathrm{M}$, snap frozen, and stored at $-80{ }^{\circ} \mathrm{C}$. These stock solutions were thawed and immediately diluted with $10 \mathrm{mM}$ Tris $\mathrm{HCl}$ buffer to a final concentration of $20 \mu \mathrm{M}$ and with a resulting $\mathrm{pH}$ of 7.3 . The peptides were injected directly into a fluid cell above a clean silicon substrate decorated with $50 \mathrm{~nm}$ silica beads. The surface was continually imaged once the peptide was injected.

\subsection{Results}

\subsubsection{Htt peptides preferentially bind to curved bilayers.}

In chapter 2, it was established that lipid bilayers covered both the curved and flat regions of the substrate. Here, these supported bilayers with both curved and flat regions were exposed to a synthetic truncated htt exon1 peptide $\left(\mathrm{Nt}^{17} \mathrm{Q}_{35} \mathrm{P}_{10} \mathrm{KK}\right)$ (Fig. 1). This peptide contained a 35 residue long polyQ domain flanked by the lipid binding $\mathrm{Nt}^{17}$ domain and a 10 residue long polyP domain. Two lysines were added at the C-terminus of the peptide to aid in solubility [37]. Observations were limited to regions of the bilayer that were confirmed to be completely covered by lipid bilayer as determined by AFM analysis, prior to exposure to $\mathrm{Nt}^{17} \mathrm{Q}_{35} \mathrm{P}_{10} \mathrm{KK}$. Addition of $\mathrm{Nt}^{17} \mathrm{Q}_{35} \mathrm{P}_{10} \mathrm{KK}$ indicated our 


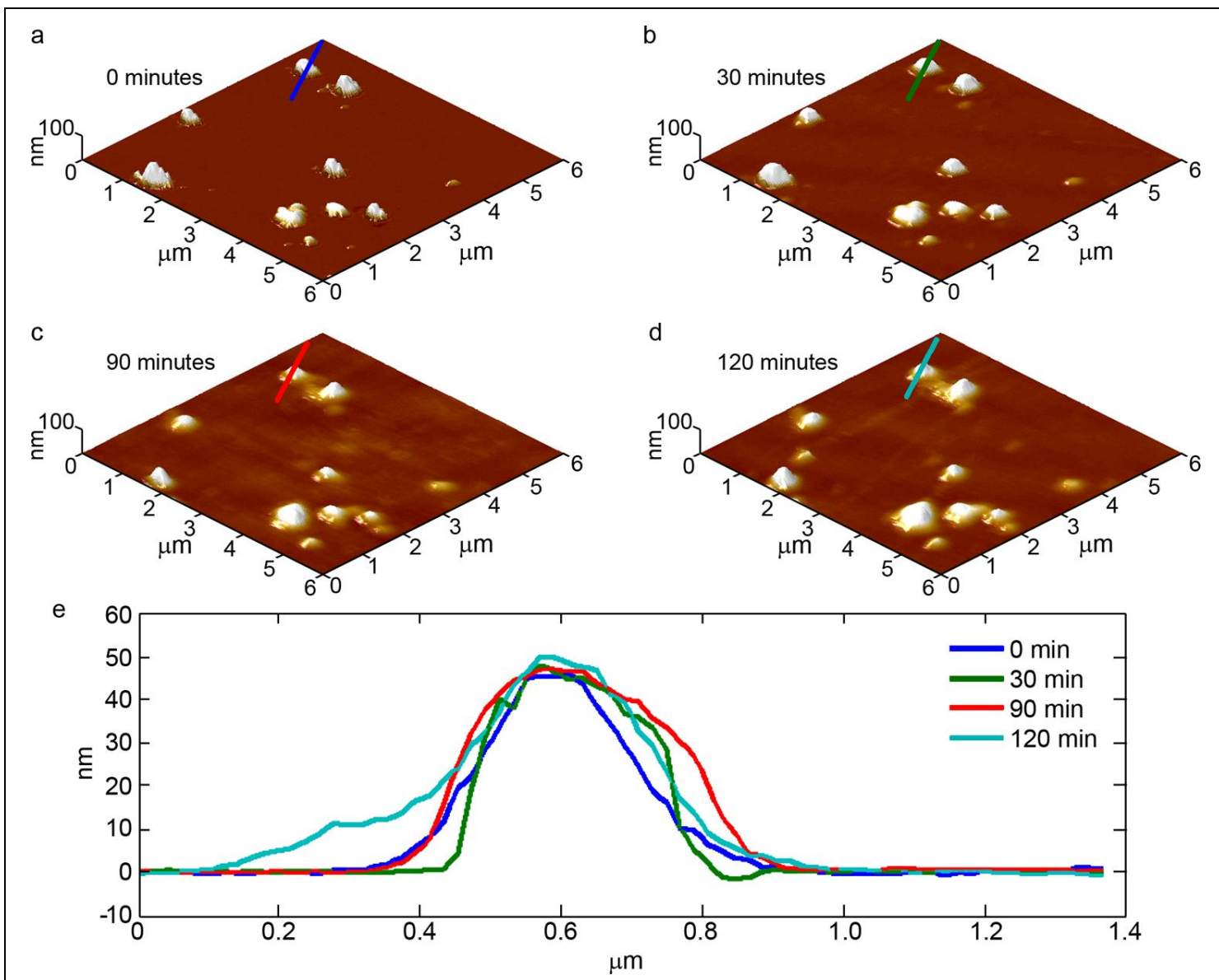

Figure 1. Time lapse AFM images taken in solution of a TBLE bilayer exposed to a synthetic htt exon1 mimicking peptide $\left(\mathrm{Nt}^{17} \mathrm{Q} 35 \mathrm{P} 10 \mathrm{KK}\right)$. Images were taken of the surface (a) before exposure $\mathrm{Nt}^{17} \mathrm{Q} 35 \mathrm{P} 10 \mathrm{KK}$ and (b-d) at 30 minute increments after injection of the peptide.

zero time-point for exposure, and no additional protein was added after this initial injection. For each experiment, $\mathrm{Nt}^{17} \mathrm{Q}_{35} \mathrm{P}_{10} \mathrm{KK}$ was freshly prepared and was in a predominately monomeric state upon initial injection; although, some small aggregates most likely were present. Control experiments exposing TBLE bilayers to protein-free vehicle solvents were performed, and no observable morphological changes in the bilayer were associated with injection of vehicle as assessed by AFM. The binding and aggregation of $\mathrm{Nt}^{17} \mathrm{Q}_{35} \mathrm{P}_{10} \mathrm{KK}$ on the bilayer surface was tracked by AFM imaging for 120 
minutes (Fig. 1). The portions of the surface occupied by beads visibly swelled within 30 minutes of exposure to $\mathrm{Nt}^{17} \mathrm{Q}_{35} \mathrm{P}_{10} \mathrm{KK}$ as peptide accumulated on the curved bilayers and these curved regions continued to expand in volume with time. The increase in volume associated with the curved regions of the substrate occurred uniformly, as all curved regions appeared to be accumulating peptide. The accumulation of peptide by the curved bilayers predominately occurred in the lateral dimension, as an increase in the width of the regions associated with beads grew more quickly in comparison with the height (see example cross sections as a function of time in Fig.6e). The flat regions of the bilayer exhibited morphological changes i.e. became rougher, in discrete regions within the first 30 minutes of exposure; however, unperturbed regions of the bilayer were still observed. With time, the area of the perturbed regions on the flat bilayers expanded and the roughness increased, this is indicative of htt binding, aggregating, and disrupting the bilayer. The height scale of the images presented in Fig.1 was optimized to demonstrate the binding of $\mathrm{Nt}^{17} \mathrm{Q}_{35} \mathrm{P}_{10} \mathrm{KK}$ to the curved bilayers. While a hint of the bilayer roughening can be observed in these images, adjusting the colormap demonstrates this more clearly (Fig. 2). A freshly formed bilayer on the flat portion of the substrate has a smooth appearance with an RMS roughness typically less than $0.2 \mathrm{~nm}$. Exposure to $\mathrm{Nt}^{17} \mathrm{Q}_{35} \mathrm{P}_{10} \mathrm{KK}$ resulted in patches of increased surface roughness on the flat lipid bilayer, consistent with disruption of the bilayer structure by binding of the peptide. The RMS roughness of these regions exceeds $0.5 \mathrm{~nm}$, and the roughness increases with time as more peptide binds and/or aggregates in these regions. 


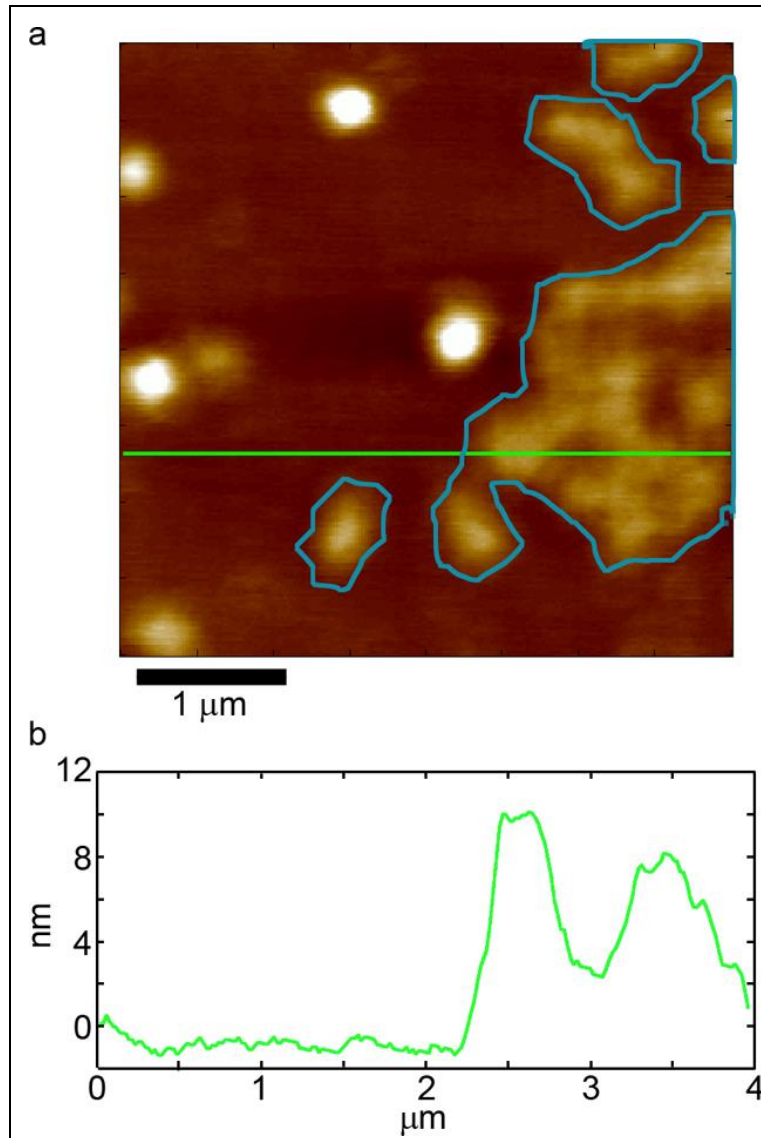

Figure 2. (a) AFM image with adjusted height contrast shows that a synthetic htt exon1 mimicking peptide $\left(\mathrm{Nt}^{17} \mathrm{Q}_{35} \mathrm{P}_{10} \mathrm{KK}\right)$ aggregates on and disrupts the flat regions of the lipid bilayer. This image was taken 90 minutes after the initial exposure to the Htt peptide. The regions indicated by blue perimeters have increased surface roughness associated with the binding and aggregation of $\mathrm{Nt}^{17} \mathrm{Q}_{35} \mathrm{P}_{10} \mathrm{KK}$. The green line in the image corresponds to the height profile in (b).
To quantify and compare the total amount of $\mathrm{Nt}^{17} \mathrm{Q}_{35} \mathrm{P}_{10} \mathrm{KK}$ that had bound to the curved and the flat regions of the bilayer respectively, the total volume change of the different regions was determined by AFM image analysis. As the flat regions of the bilayer represented a significantly larger surface area of the substrate, the change in volume was normalized per unit area. The initial volume of each lipid covered bead before exposure to $\mathrm{Nt}^{17} \mathrm{Q}_{35} \mathrm{P}_{10}\left(V_{0}\right)$ was subtracted for each subsequent measurements $\left(V_{t}\right)$ to determine the volume change $(\Delta V)$ associated with deposition of peptide on curved bilayers at any given time. However, there are contributions to the measured volume of objects measured by AFM due to the finite size and shape of the probe tip, often referred to as tip

convolution. This results in the measured volume of any given object in an AFM image being larger than the true volume of that object. Matlab based simulations of tip convolution [38] associated with AFM imaging of model beads that systematically increased in volume were performed to determine the expected error associated with the 


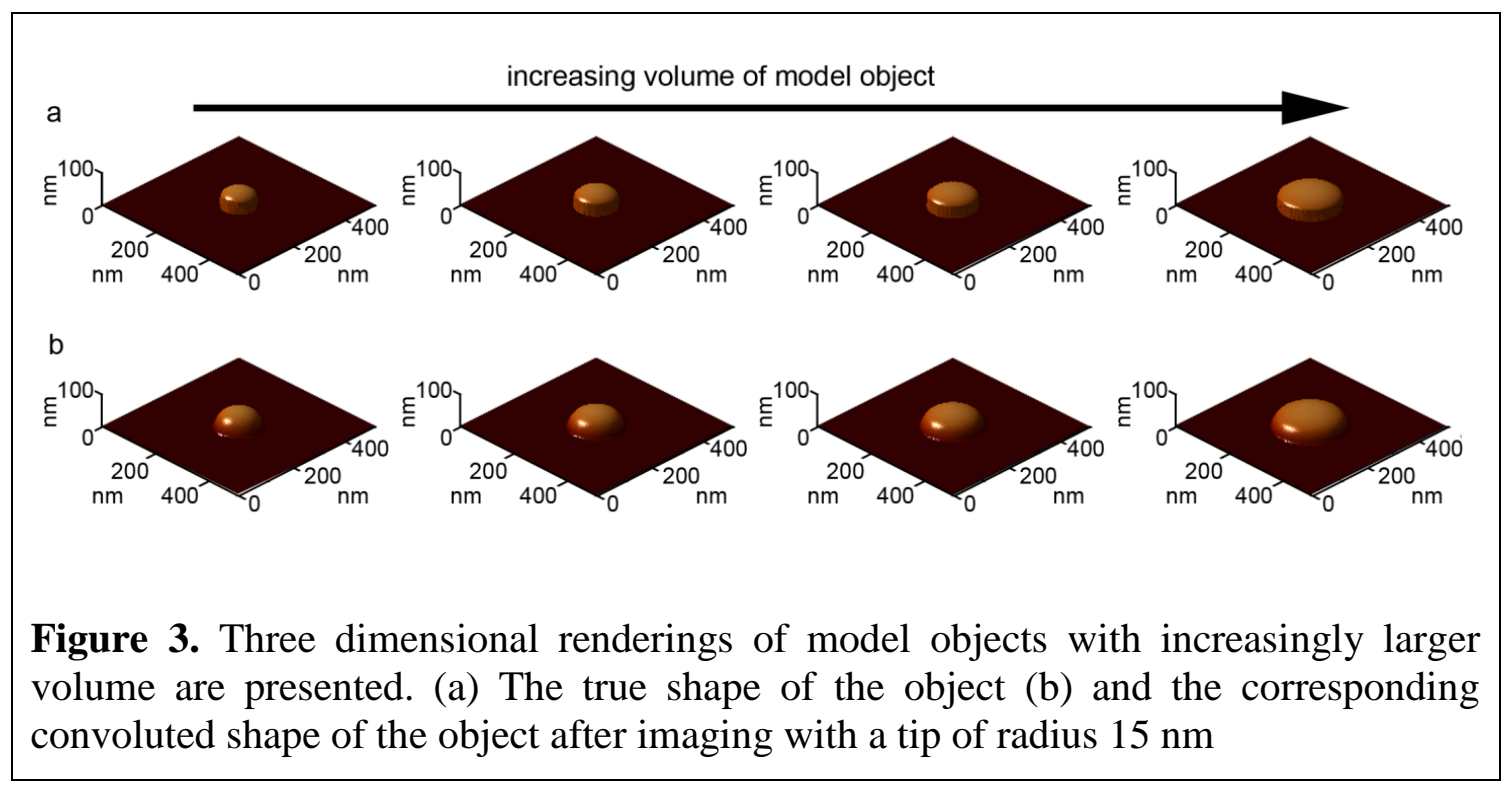

finite size and shape of the tip in determining the volume of deposited peptide. On this model, the volume change $(\Delta V)$ associated with deposition of peptide on curved bilayers at any given time point was determined by:

$\Delta V=V_{0}-V_{t}$

A model $50 \mathrm{~nm}$ bead (approximated as a sphere, original object) was immobilized on a 3D surface (Fig. 3a). The volume of this bead was systematically increased (expanded objects) to simulate the accumulation of peptide on a curved membrane supported by a bead. This was done in such a way that most of the volume was added in the lateral dimension as opposed to the vertical, which is similar to what was observed when exposing curved bilayers to peptide in experiment (Fig. 1e). As the exact volume of these model objects is known, the \% increase in the true volume of the feature can be determined (table 1): 
Increase in true volume $=\left(\frac{\text { volume of expanded object-volume of original object }}{\text { volume of original object }}\right) * 100$

Table 1

\begin{tabular}{ccc}
\hline $\begin{array}{c}\text { \% increase in true } \\
\text { volume }\end{array}$ & $\begin{array}{c}\text { \% contribution due to } \\
\text { convolution }\end{array}$ & volume change \\
\hline 0.0 & 43.1 & 0.0 \\
6.0 & 40.4 & -3.4 \\
11.4 & 39.3 & 2.6 \\
15.9 & 38.9 & 7.7 \\
21.9 & 37.0 & 9.5 \\
44.5 & 33.7 & 12.6 \\
70.1 & 30.0 & 11.4 \\
97.2 & 27.4 & 11.3 \\
126.8 & 24.9 & 10.6 \\
208.3 & 20.7 & 9.4 \\
302.0 & 17.8 & \\
\hline
\end{tabular}

Model AFM tips were constructed as hemispheres with varying radii. Results presented here are for a $15 \mathrm{~nm}$ tip. These tips were used to virtually image the model surfaces to obtain simulated AFM images with tip convolution (Fig. 8b). The $\%$ contribution due to tip convolution for each model object was calculated as: 
$\%$ contribution due to tip convolution $=\frac{V_{t}-V_{\text {true }}}{V_{\text {true }}} \times 100$.

where $V_{t}$ is the measured volume after tip convolution and $V_{\text {true }}$ is the true volume of the model object. Using equation 1 , the true volume change and the measured volume change after tip convolution associated with our model objects was determined. The $\%$ error in the measured volume increase was calculated as follows:

Measured volume error $=\frac{\Delta V_{\text {with convolution }}-\Delta V_{\text {true }}}{\Delta V_{\text {true }}} \times 100$

where $\Delta V_{\text {with convolution }}$ is the change in volume measured after tip convolution and $\Delta V_{\text {true }}$ is the true change in volume. In short, these simulations indicated that while tip convolution caused the measured volume to typically be overestimated, this error never exceeded $15 \%$. The increased volume associated with flat regions of the bilayer was measured by summing up the volume of features observed above the baseline of the unperturbed bilayer height. By both of these measurements, the volume associated with the initial deposition of bilayer was controlled for both the curved and flat regions of the surface. Based on this analysis, $\mathrm{Nt}^{17} \mathrm{Q}_{35} \mathrm{P}_{10} \mathrm{KK}$ preferentially accumulated on the curved rather than the flat regions of the bilayer, even when taking into account the potential $15 \%$ overestimation due to tip convolution of the beads (Fig. 4). Furthermore, shading has been added to Fig. 4 to indicate what the volume change associated with peptide deposition would be if there was a $15 \%$ overestimation, and this value was still significantly larger when compared to the amount of peptide deposited on flat regions of the bilayer. While $\mathrm{Nt}^{17} \mathrm{Q}_{35} \mathrm{P}_{10} \mathrm{KK}$ accumulated on both types of surfaces, significant 


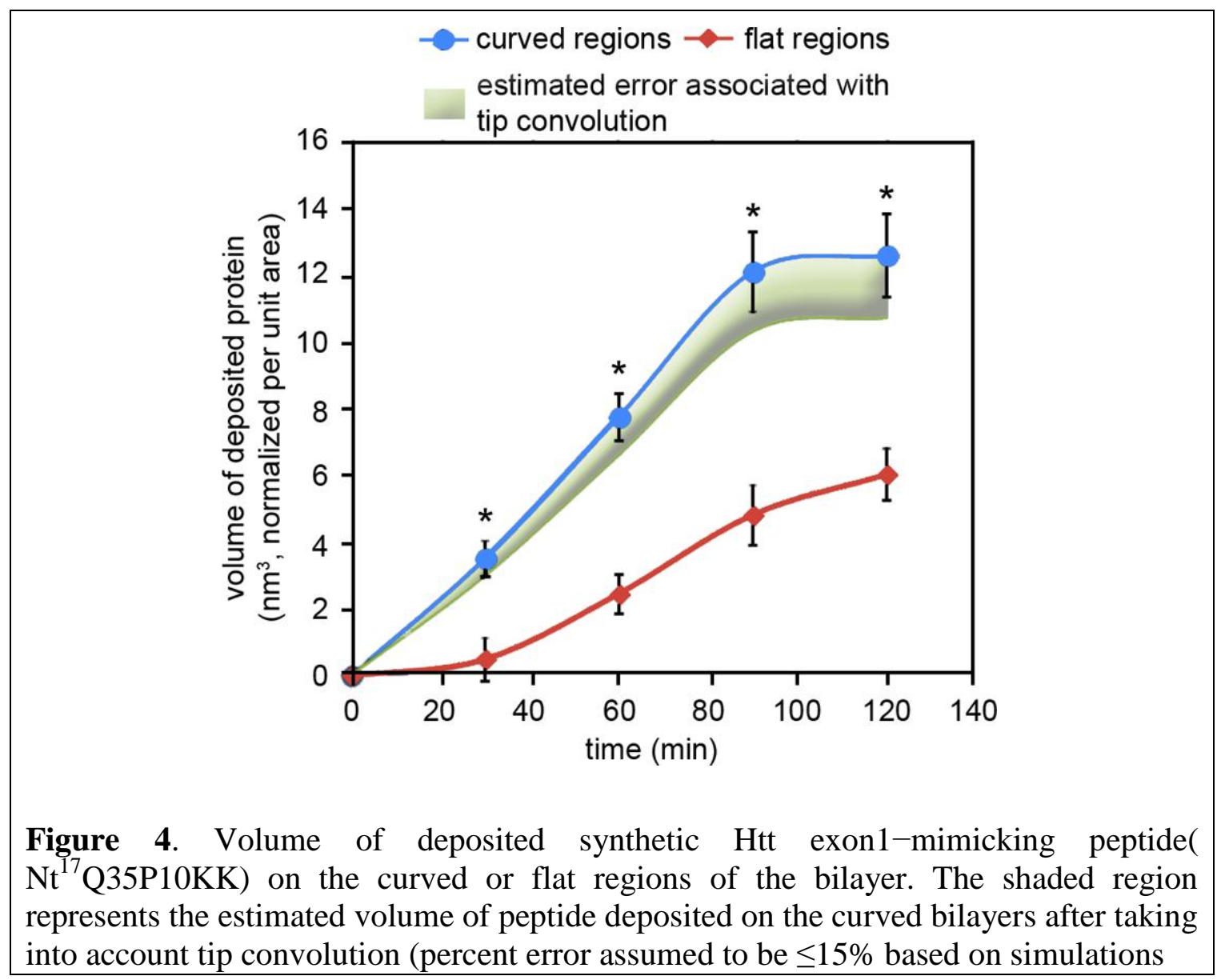

differences $(p<0.05)$ in the volume of deposited peptide were observed within 30 minutes. The amount of deposited peptide increased linearly for the next hour for both regions of the bilayer; however, the accumulation of peptide occurred more rapidly on the curved regions. After $\sim 90$ minutes, accumulation of peptide on the curved bilayer appeared to level off. 


\subsection{Discussion}

While tapping mode AFM in solution is widely used to study morphological and mechanical properties of supported lipid bilayers, one of the limitations associated with comparing these results to physiologically relevant membranes is the lack of membrane curvature associated with such systems. A potential method to induce curvature in supported lipid membranes is introducing local curvature in the substrate through depositing silica nanobeads onto a silicon substrate, and by the process of vesicle fusion, as discussed in detail in chapter 2.

When exposing these model bilayers to freshly prepared solutions of an $\mathrm{N}$ terminal htt fragment, it was found that the peptide preferentially accumulated on curved regions of the bilayer. While htt fragments with expanded polyQ domains are predominately found in nuclear or cytoplasmic inclusion bodies, [7] htt also directly associates with numerous membranous surfaces, such as mitochondria, endoplasmic reticulum, tubulovesicles, endosomes, lysosomes and synaptic vesicles, within the cell [2, 39]. The environments provided by these membrane surfaces could influence the aggregation of htt with expanded polyQ domains, and these membranous structures exhibit varying degrees of curvature. This curvature may provide nucleation sites for aggregation by creating local areas of high htt concentration due to enhanced binding. The accumulation of monomeric htt and/or pre-fibrillar aggregates directly binding lipid membranes has been shown to disrupt bilayer integrity and induce mechanical changes in the membrane $[14,15,26]$.

Beyond these implications of htt/lipid interactions in disease mechanisms, there are several membrane-associated functions attributable to htt in which the lipid binding 
properties of $\mathrm{Nt}^{17}$ may play a role. Some of these functions include htt functioning in cellular adhesion, [40] motility, [41, 42] cholesterol and energy homeostasis, [43] as a molecular scaffold for coordination of membrane and cytoskeletal communication, [8] and facilitating micrutobule-dependent vesicle transport [44]. $\mathrm{Nt}^{17}$ facilitates the trafficking of htt exon1 to membranes associated with the ER, autophagic vacuoles, mitochondria, and Golgi $[22,23]$. Membrane curvature sensing by the $\mathrm{Nt}^{17}$ domain of htt may represent a mechanistic detail of some of these functions, and disruption of these normal lipid associated functions of htt may play a role in toxicity associated with expanded polyQ domains. Supporting the notion that $\mathrm{Nt}^{17}$ directly interacts with lipid membranes, the binding of htt exon1 to large unilamellar vesicles comprised of PC or a mixture of PC and PS alters the helical content of the $\mathrm{Nt}^{17}$, and the associated changes in helical content could be inhibited by an addition of a proline at the $8^{\text {th }}$ residue of $\mathrm{Nt}^{17}$ [22]. Structural studies utilizing solid state NMR suggest that $\mathrm{Nt}^{17}$ has a predominantly random coil structure in solution and adopts its $\alpha$-helical structure upon binding lipid bilayers $[9,10]$. Furthermore, the presence of $\mathrm{Nt}^{17}$ in synthetic polyQ peptides was necessary to bind model TBLE membranes and induce leakage of TBLE vesicles [15].

One potential mechanism to explain how $\mathrm{Nt}^{17}$, which is an $\mathrm{AH}$, preferentially binds curved membranes is that bilayer curvature introduces a high density of membrane defects that can act as binding sites. AHs represent a common motif in a variety of proteins to sense membrane curvature [27-29]. The insertion process of AHs may be facilitated by lipid packing defects induced by the membrane curvature, resulting in a higher affinity of the protein for positively curved membranes [28, 45, 46]. However, recent studies have suggested that the curvature-selective binding of some AHs is due to 
intrinsic properties of the membrane, that is, a higher density of binding sites rather than a higher affinity of the protein for the membrane [29]. Assuming that the lipid mass is conserved on both leaflets of a lipid bilayer and that the lipid composition of the bilayer remains constant, the density of defects within the leaflet of a bilayer associated with positive curvature would increase with bending [29]. Once a htt monomer (or possibly small aggregate) binds to the lipid membrane, this creates a site where more htt could preferentially accumulate through interaction with the immobilized bound peptide. This notion is supported by previous observations that monomeric htt exon1 proteins preferentially accumulate onto immobilized pre-formed aggregates, [47] and that htt exon1 tends to alter the morphology of lipid bilayers in discrete regions that grow in size with time $[14,15]$. These discrete regions of disruption on the flat portions of the bilayer were also observed in this study. The binding of amyloid-forming proteins to membrane defects via AHs has been demonstrated for other amyloid-forming proteins, such as $\alpha$ synuclein [46, 48] and islet amyloid polypeptide [49].

While the data presented here suggests that the presence of bilayer defects induced by curvature can enhance the initial binding and accumulation of htt on membranes, several other factors can potentially play a role in regulating the interaction of htt exon1 fragments facilitated by $\mathrm{Nt}^{17}$ with lipid membranes. For example, several residues in $\mathrm{Nt}^{17}$ can undergo post translational modifications, such as phosphorylation, acetylation, and SUMOlyation, and some of these modifications have been shown to regulate the interaction of htt with specific organelles [22, 50]. Additional affinity-based components to the interaction of other AHs with lipid bilayers have been experimentally observed. Such considerations include electrostatics, [51, 52] partitioning of lipid 
components,[53-55] or the presence of other specific recognition motifs [56, 57]. In particular, electrostatic mechanisms likely play a role in htt/lipid interactions, as htt binds multivalent phosphatidylinsoitol phospates (PIPs) and has a high affinity for cardiolipin $[25,58]$.

\subsection{Conclusion}

Apart from the lipid membrane curvature, it has become increasingly evident that there are several that determine the propensity of polyQ proteins to aggregate. Various factors, including posttranslational modifications may impact polyQ-aggregation process and its subsequent toxicity. Specifically, htt undergoes a variety of posttranslational modifications [59]. Acetylation and phosphorylation of threonine-3 are the most prevalent modifications of htt exon 1, which greatly impacts the aggregation of htt in cells and in vivo [60]. With AFM as robust imaging tool, detailed insights on the kinetics of posttranslational modifications on htt exon1 aggregation and the morphological characteristics of the resulting aggregates can be obtained. The impact of acetylation modification on htt aggregation is the focus of the next chapter.

\subsection{References}

1. Group, T.H.S.D.C.R., A novel gene containing a trinucleotide repeat that is expanded and unstable on Huntington's disease chromosomes. Cell, 1993. 72(6): p. $971-983$. 
2. DiFiglia, M., et al., Aggregation of huntingtin in neuronal intranuclear inclusions and dystrophic neurites in brain. Science, 1997. 277(5334): p. 1990-1993.

3. Ravina, B., et al., The relationship between CAG repeat length and clinical progression in Huntington's disease. Movement Disorders, 2008. 23(9): p. 12231227.

4. Kim, Y.J., et al., Caspase 3-cleaved N-terminal fragments of wild-type and mutant huntingtin are present in normal and Huntington's disease brains, associate with membranes, and undergo calpain-dependent proteolysis. Proceedings of the National Academy of Sciences of the United States of America, 2001. 98(22): p. 12784-12789.

5. Ratovitski, T., et al., Mutant Huntingtin N-terminal Fragments of Specific Size Mediate Aggregation and Toxicity in Neuronal Cells. Journal of Biological Chemistry, 2009. 284(16): p. 10855-10867.

6. Ratovitski, T., et al., N-terminal proteolysis of full-length mutant huntingtin in an inducible PC12 cell model of Huntington's disease. Cell Cycle, 2007. 6(23): p. 2970-2981.

7. Davies, S.W., et al., Formation of neuronal intranuclear inclusions underlies the neurological dysfunction in mice transgenic for the HD mutation. Cell, 1997. 90(3): p. 537-548.

8. Kegel, K.B., et al., Huntingtin associates with acidic phospholipids at the plasma membrane. Journal of Biological Chemistry, 2005. 280(43): p. 36464-36473. 
9. Michalek, M., E.S. Salnikov, and B. Bechinger, Structure and Topology of the Huntingtin 1-17 Membrane Anchor by a Combined Solution and Solid-State NMR Approach. Biophysical Journal, 2013. 105(3): p. 699-710.

10. Michalek, M., et al., Membrane Interactions of the Amphipathic Amino Terminus of Huntingtin. Biochemistry, 2013. 52(5): p. 847-858.

11. Jayaraman, M., et al., Slow Amyloid Nucleation via alpha-Helix-Rich Oligomeric Intermediates in Short Polyglutamine-Containing Huntingtin Fragments. Journal of Molecular Biology, 2012. 415(5): p. 881-899.

12. Sivanandam, V.N., et al., The Aggregation-Enhancing Huntingtin N-Terminus Is Helical in Amyloid Fibrils. Journal of the American Chemical Society, 2011. 133(12): p. 4558-4566.

13. Thakur, A.K., et al., Polyglutamine disruption of the huntingtin exon $1 \mathrm{~N}$ terminus triggers a complex aggregation mechanism. Nature Structural \& Molecular Biology, 2009. 16(4): p. 380-389.

14. Burke, K.A., et al., Huntingtin disrupts lipid bilayers in a polyQ-length dependent manner. Biochimica Et Biophysica Acta-Biomembranes, 2013. 1828(8): p. 19531961.

15. Burke, K.A., et al., The Interaction of Polyglutamine Peptides with Lipid Membranes Is Regulated by Flanking Sequences Associated with Huntingtin. Journal of Biological Chemistry, 2013. 288(21): p. 14993-15005.

16. Mishra, R., et al., Inhibiting the Nucleation of Amyloid Structure in a Huntingtin Fragment by Targeting alpha-Helix-Rich Oligomeric Intermediates. Journal of Molecular Biology, 2012. 415(5): p. 900-917. 
17. Bhattacharyya, A., et al., Oligoproline effects on polyglutamine conformation and aggregation. Journal of Molecular Biology, 2006. 355(3): p. 524-535.

18. Darnell, G., et al., Flanking polyproline sequences inhibit beta-sheet structure in polyglutamine segments by inducing PPII-like helix structure. Journal of Molecular Biology, 2007. 374(3): p. 688-704.

19. Kegel, K.B., et al., Huntingtin expression stimulates endosomal-lysosomal activity, endosome tubulation, and autophagy. Journal of Neuroscience, 2000. 20(19): p. 7268-7278.

20. Suopanki, J., et al., Interaction of huntingtin fragments with brain membranes clues to early dysfunction in Huntington's disease. Journal of Neurochemistry, 2006. 96(3): p. 870-884.

21. Valencia, A., et al., Mutant Huntingtin and Glycogen Synthase Kinase 3-beta Accumulate in Neuronal Lipid Rafts of a Presymptomatic Knock-In Mouse Model of Huntington's Disease. Journal of Neuroscience Research, 2010. 88(1): p. 179190.

22. Atwal, R.S., et al., Huntingtin has a membrane association signal that can modulate huntingtin aggregation, nuclear entry and toxicity. Human Molecular Genetics, 2007. 16(21): p. 2600-2615.

23. Rockabrand, E., et al., The first 17 amino acids of Huntingtin modulate its subcellular localization, aggregation and effects on calcium homeostasis. Human Molecular Genetics, 2007. 16(1): p. 61-77. 
24. Trettel, F., et al., Dominant phenotypes produced by the HD mutation in STHdh(Q111) striatal cells. Human Molecular Genetics, 2000. 9(19): p. 27992809.

25. Kegel, K.B., et al., Polyglutamine expansion in huntingtin increases its insertion into lipid bilayers. Biochemical and Biophysical Research Communications, 2009. 387(3): p. 472-475.

26. Burke, K.A., E.A. Yates, and J. Legleiter, Amyloid-Forming Proteins Alter the Local Mechanical Properties of Lipid Membranes. Biochemistry, 2013. 52(5): p. 808-817.

27. Drin, G. and B. Antonny, Amphipathic helices and membrane curvature. FEBS Letters, 2010. 584(9): p. 1840-1847.

28. Drin, G., et al., A general amphipathic alpha-helical motif for sensing membrane curvature. Nature Structural \& Molecular Biology, 2007. 14(2): p. 138-146.

29. Hatzakis, N.S., et al., How curved membranes recruit amphipathic helices and protein anchoring motifs. Nature Chemical Biology, 2009. 5(11): p. 835-841.

30. Kim, M.W., et al., Secondary Structure of Huntingtin Amino-Terminal Region. Structure, 2009. 17(9): p. 1205-1212.

31. Drolle, E., Ravi M. Gaikwad, and Z. Leonenko, Nanoscale Electrostatic Domains in Cholesterol-Laden Lipid Membranes Create a Target for Amyloid Binding. Biophysical Journal, 2012. 103(4): p. L27-L29.

32. Hane, F., E. Drolle, and Z. Leonenko, Effect of cholesterol and amyloid- $\beta$ peptide on structure and function of mixed-lipid films and pulmonary surfactant BLES: an 
atomic force microscopy study. Nanomedicine: Nanotechnology, Biology and Medicine, 2010. 6(6): p. 808-814.

33. Legleiter, J., et al., The Modulating Effect of Mechanical Changes in Lipid Bilayers Caused by ApoE-Containing Lipoproteins on A beta Induced Membrane Disruption. Acs Chemical Neuroscience, 2011. 2(10): p. 588-599.

34. Yates, E.A., et al., Specific Domains of A beta Facilitate Aggregation on and Association with Lipid Bilayers. Journal of Molecular Biology, 2013. 425(11): p. 1915-1933.

35. Yip, C.M., A.A. Darabie, and J. McLaurin, A beta 42-peptide assembly on lipid Bilayers. Journal of Molecular Biology, 2002. 318(1): p. 97-107.

36. Yip, C.M., et al., Cholesterol, a modulator of membrane-associated A betafibrillogenesis and neurotoxicity. Journal of Molecular Biology, 2001. 311(4): p. 723-734.

37. Chen, S.M. and R. Wetzel, Solubilization and disaggregation of polyglutamine peptides. Protein Science, 2001. 10(4): p. 887-891.

38. Villarrubia, J.S., Algorithms for scanned probe microscope image simulation, surface reconstruction, and tip estimation. Journal of Research of the National Institute of Standards and Technology, 1997. 102(4): p. 425-454.

39. Gutekunst, C.A., et al., Nuclear and neuropil aggregates in Huntington's disease: relationship to neuropathology. J Neurosci, 1999. 19(7): p. 2522-34.

40. Strehlow, A.N., J.Z. Li, and R.M. Myers, Wild-type huntingtin participates in protein trafficking between the Golgi and the extracellular space. Hum Mol Genet, 2007. 16(4): p. 391-409. 
41. Ritch, J.J., et al., Multiple phenotypes in Huntington disease mouse neural stem cells. Molecular and Cellular Neuroscience, 2012. 50(1): p. 70-81.

42. Myre, M.A., et al., Deficiency of huntingtin has pleiotropic effects in the social amoeba Dictyostelium discoideum. PLoS Genet, 2011. 7(4): p. e1002052.

43. Jacobsen, J.C., et al., HD CAG-correlated gene expression changes support a simple dominant gain of function. Hum Mol Genet, 2011. 20(14): p. 2846-60.

44. Caviston, J.P. and E.L. Holzbaur, Huntingtin as an essential integrator of intracellular vesicular trafficking. Trends Cell Biol, 2009. 19(4): p. 147-55.

45. Cornell, R.B. and S.G. Taneva, Amphipathic helices as mediators of the membrane interaction of amphitropic proteins, and as modulators of bilayer physical properties. Current Protein \& Peptide Science, 2006. 7(6): p. 539-552.

46. Nuscher, B., et al., alpha-synuclein has a high affinity for packing defects in a bilayer membrane - A thermodynamics study. Journal of Biological Chemistry, 2004. 279(21): p. 21966-21975.

47. Legleiter, J., et al., Mutant Huntingtin Fragments Form Oligomers in a Polyglutamine Length-dependent Manner in Vitro and in Vivo. Journal of Biological Chemistry, 2010. 285(19): p. 14777-14790.

48. Davidson, W.S., et al., Stabilization of alpha-synuclein secondary structure upon binding to synthetic membranes. Journal of Biological Chemistry, 1998. 273(16): p. 9443-9449.

49. Caillon, L., O. Lequin, and L. Khemtemourian, Evaluation of membrane models and their composition for islet amyloid polypeptide-membrane aggregation. Biochimica Et Biophysica Acta-Biomembranes, 2013. 1828(9): p. 2091-2098. 
50. Xia, J.R., et al., Huntingtin contains a highly conserved nuclear export signal. Human Molecular Genetics, 2003. 12(12): p. 1393-1403.

51. McLaughlin, S. and D. Murray, Plasma membrane phosphoinositide organization by protein electrostatics. Nature, 2005. 438(7068): p. 605-611.

52. Yeung, T., et al., Membrane phosphatidylserine regulates surface charge and protein localization. Science, 2008. 319(5860): p. 210-213.

53. Linder, M.E. and R.J. Deschenes, Palmitoylation: policing protein stability and traffic. Nature Reviews Molecular Cell Biology, 2007. 8(1): p. 74-84.

54. Manneville, J.-B., et al., COPI coat assembly occurs on liquid-disordered domains and the associated membrane deformations are limited by membrane tension. Proceedings of the National Academy of Sciences of the United States of America, 2008. 105(44): p. 16946-16951.

55. Rocks, O., et al., An acylation cycle regulates localization and activity of palmitoylated Ras isoforms. Science, 2005. 307(5716): p. 1746-1752.

56. Lemmon, M.A., Membrane recognition by phospholipid-binding domains. Nature Reviews Molecular Cell Biology, 2008. 9(2): p. 99-111.

57. McMahon, H.T. and J.L. Gallop, Membrane curvature and mechanisms of dynamic cell membrane remodelling. Nature, 2005. 438(7068): p. 590-596.

58. Kegel-Gleason, K.B., Huntingtin Interactions with Membrane Phospholipids: Strategic Targets for Therapeutic Intervention? Journal of Huntington's Disease, 2013. 2(3): p. 239-250.

59. Hands, S.L. and A. Wyttenbach, Neurotoxic protein oligomerisation associated with polyglutamine diseases. Acta Neuropathologica, 2010. 120(4): p. 419-437. 
60. Aiken, C.T., et al., Phosphorylation of Threonine 3 Implications for huntingtin aggregation and neurotoxicity. Journal of Biological Chemistry, 2009. 284(43): p. 29427-29436. 


\section{Acetylation within the first 17 amino acids of huntingtin exon1 alters aggregation and binding to lipid membranes ${ }^{1}$}

Huntington disease (HD) is a genetic neurodegenerative disorder caused by an expanded polyglutamine (polyQ) domain near the N-terminus of the huntingtin (htt) protein. Expanded polyQ directly leads to htt aggregation. The first 17 amino acids $\left(\mathrm{Nt}^{17}\right)$ in htt is a lipid-binding domain that also promotes diverse aggregate species of htt. $\mathrm{Nt}^{17}$ undergoes a number of posttranslational modifications that can modulate htt's toxicity, subcellular localization, and trafficking of vesicles. N-terminal acetylation of htt has been implicated in the etiology of HD. Given the importance of acetylation in HD, this study sought to evaluate the impact of lysine acetylation on htt aggregation in solution and on model lipid bilayers. Acetylation of htt-exon1(51Q), and synthetic truncated htt exon1 mimicing peptides $\left(\mathrm{Nt}^{17} \mathrm{Q}_{35} \mathrm{P}_{10} \mathrm{KK}\right)$ was achieved using a selective covalent label sulfo-Nhydroxysuccinimide (NHSA) in molar ratios of $1 \mathrm{x}, 2 \mathrm{x}$, and 3x NHSA per peptide. With this treatment, all three lysine residues $(\mathrm{K} 6, \mathrm{~K} 9$, and $\mathrm{K} 15)$ in $\mathrm{Nt}^{17}$ were significantly labeled. N-terminal htt acetylation decreased fibril formation in solution and promoted the formation of larger globular aggregates. Acetylation strongly altered htt's ability to bind lipid membranes. Acetylated htt bound lipid membranes and disrupted lipid bilayer morphology less aggressively compared to unlabeled htt. Mechanistic insights into how acetylation alters the interaction of $\mathrm{Nt}^{17}$ with lipid membranes were provided by computational studies. The results presented here, highlight that $\mathrm{N}$-terminal acetylation influences the aggregation of htt and its interaction with lipid bilayers.

\footnotetext{
${ }^{1}$ This chapter is based on submitted manuscript "Acetylation alters huntingtin aggregation and membranes"
} 


\subsection{Introduction: Huntingtin's disease and post translational modifications.}

Huntington's disease (HD) is a neurodegenerative disorder caused by an expanded CAG trinucleotide repeat in the IT15 gene that codes for the huntingtin (htt) protein. This CAG expansion encodes an abnormal stretch of more than 35-40 polyglutamine (polyQ) repeats within the first exon of htt [1]. In HD, the age of onset and severity in disease is highly correlated with polyQ length [2, 3]. Expanded polyQ domains in htt are directly implicated in the aggregation of htt into fibrils and a variety of other structures [4-6]. The polyQ domain is located in the first exon of htt, beginning with the $18^{\text {th }}$ residue, and substantial evidence points to $\mathrm{N}$-terminal fragments of htt comparable to exon 1 being involved in HD [7-12].

In HD patients' brains, mutant htt is detected predominantly in microscopic inclusion bodies in the cytoplasm and nucleus [10], but it also associates with membranous organelles, including mitochondria, endoplasmic reticulum (ER), tubulovesicles, endosomes, lysosomes and synaptic vesicles [13-16]. Approximately half of endogenous htt partitions with membranes after subcellular fractionation of neuronlike clonal striatal cells [17]. Futhermore, membraneous structures are assimilated onto the surfaces of htt inclusion bodies in cellular models [17, 18], and htt aggregates accumulate brain lipids in mouse models of $\operatorname{HD}[19,20]$. With regards to N-terminal fragments of htt, $\mathrm{Nt}^{17}$ is required for membrane localization [21, 22] and regulates lipid binding [23]. Beyond functioning in lipid binding, $\mathrm{Nt}^{17}$ promotes oligomer formation [24, 25], and targeting $\mathrm{Nt}^{17}$ directly is an effective strategy for inhibiting aggregation $[24,26]$.

In solution, monomeric $\mathrm{Nt}^{17}$ is predominately disordered, as demonstrated by NMR [25], but circular dichroism (CD) studies suggest that $\mathrm{Nt}^{17}$ contains $10-50 \% \alpha$ - 
helical content, depending on buffer conditions $[21,25,27,28]$. This suggests that $\mathrm{Nt}^{17}$ may adopt multiple configurations. Such a notion is supported by computational studies, some of which predict that $\mathrm{Nt}^{17}$ can form an $\alpha$-helix in aqueous solution [29, 30]; others suggest that $\mathrm{Nt}^{17}$ is predominately unstructured in solution [28, 31-34]. However, the $\mathrm{Nt}^{17}$ domain appears to be $\alpha$-helical in at least some aggregate forms of htt $[26,35,36]$, and it transitions to an $\alpha$-helical structure upon self-association [24] or when it interacts with phospholipids, detergents, or other apolar compounds [21, 25, 27, 37]. Furthermore, recent IMS-MS experiments suggest the presence of coexisting solution conformers for $\mathrm{Nt}^{17}$ peptides [38].

Several post translational modifications (PTMs) including proteolytic cleavage, acetylation, phosphorylation, ubiquitination, and SUMOylation, are associated with HD pathogenesis [39-41]. Dysregulation of PTMs triggered by expanded polyQ tracts may lead to aberrant htt interactions [42]. A number of PTM sites are located in the $\mathrm{Nt}^{17}$ domain, with some sites associated with multiple modifications [43]. Specifically, proteomic mapping by mass spectrometry (MS) identified acetylation sites at K6, K9, and K15 [44]. This N-terminal acetylation preferentially occurred in htt with polyQ lengths below the disease threshold, suggesting that decreased acetylation of expanded htt could play a role in pathogenic mechanisms associated with HD.

Membrane related changes are a clear biochemical feature of HD [45-47]. Htt predominately localizes to a variety of membranous surfaces within cells, and its strong association with lipids may play a prominent role in the normal function of htt $[45,48]$. Amino-terminal mutant htt fragments have been shown to aggregate on and damage a variety of phospholipid bilayers, suggesting that phospholipid interactions may play a 
role htt-related toxicity $[19,23,49]$. As a result, determining factors that regulate the affinity of htt for membranes is critical in understanding not only the normal functions of htt, but also in identifying ways to modify htt/lipid interactions for potential therapeutic strategies. It is clear that $\mathrm{Nt}^{17}$ influences the aggregation of $\mathrm{N}$-terminal fragments of htt $[24,26,35]$ and their direct interactions with lipid membranes [23, 27, 37, 50, 51]. Furthermore, PTMs within $\mathrm{Nt}^{17}$ have a profound effect not only on htt function and translocation [52-54], but also on the toxicity associated with mutant htt containing expanded polyQ domains [42, 43, 55-57]. However, the impact of acetylation of $\mathrm{Nt}^{17}$ on modulating htt aggregation and its interactions with lipid bilayers is poorly understood. The aim here is to elucidate the influence of acetylation of $\mathrm{Nt}^{17}$ on these phenomena.

\subsection{Materials and Methods}

\subsubsection{Peptide preparation.}

Synthetic $\mathrm{Nt}^{17} \mathrm{Q}_{35} \mathrm{P}_{10} \mathrm{KK}$ was purchased from the Keck institute (Yale University) and stored at $-20{ }^{\circ} \mathrm{C}$ until later use. The synthetic, model htt peptide used in this study contains 35 glutamines (which is sufficient for aggregation), [58] a C-terminal polyproline (polyP) domain that is ten residues in length, and the previously mentioned $\mathrm{Nt}^{17}$ on the N-terminus. Disaggregation and solubilization of peptides were achieved by a variation on published protocols [59]. In short, 1.0-mg portions were dissolved in $1.00 \mathrm{ml}$ of 50/50 trifluoroacetic acid/hexafluoroisopropanol (TFA/HFIP) to ensure disaggregation. The samples were aliquoted into $250-\mu 1$ portions and dried under a steady stream of nitrogen until most of the TFA/HFIP was removed. The aliquots were then placed in a centrifugal evaporator for $3 \mathrm{~h}$ to ensure removal of all TFA/HFIP. The 4. Acetylation alters huntingtin aggregation and membranes 
resulting $0.250-\mathrm{mg}$ peptide films were stored at $-20^{\circ} \mathrm{C}$ until further use. The peptide films were reconstituted in $\mathrm{pH} 3$ TFA, and then diluted to $20 \mu \mathrm{M}$ in $\mathrm{pH} 7 \mathrm{PBS}$. Labeling was performed immediately after reconstitution at the three molar ratios. The labeling reaction was quenched by the addition of $100 \mu \mathrm{L}$ Tris $\mathrm{HCl}$ after a one-minute reaction time. $50 \mu \mathrm{L}$ of acetonitrile was added to each sample to prevent aggregation before enzymatic digestion. $2.0 \mu \mathrm{L}$ of $1.0 \mathrm{mg} / \mathrm{mL}$ chymotrypsin was added to each sample and allowed to digest overnight at $37^{\circ} \mathrm{C}$. Digestion was quenched by addition of $5 \mu \mathrm{L}$ of formic acid. Samples were aliquoted and immediately analyzed by LC-MS/MS.

\subsubsection{Liquid Chromatography/Tandem Mass Spectrometry.}

LC-MS/MS was performed using an Accela binary pump LC coupled to a $\mathrm{Q}$ Exactive mass spectrometer (Thermo Scientific, San Jose, CA). Separation was facilitated using a Synergi Fusion RP-80 2.0 x 100 mm column, particle size of 4 micron (Phenomenex, Torrance, CA) with water $0.1 \%$ formic acid as solvent $\mathrm{A}$, and acetonitrile with $0.1 \%$ formic acid as solvent B. A 60 minute gradient was used (5-100\% B over 60 minutes) with a ten minute column re-equilibration between successive runs, at a flow rate of $0.200 \mathrm{~mL} / \mathrm{min}$. A $20 \mu \mathrm{L}$ sample injection was used for all injections. The mass spectrometer was operated in a data-dependent mode, where the 5 most intense ions in each scan were submitted for MS/MS HCD fragmentation. Data analysis was performed using Thermo Proteome Discoverer (Thermo Scientific). A database containing only the peptide of interest was generated manually and used as search criteria. The program searched for both acetylated and unmodified peptide ions of interest within each LC- 
MS/MS dataset. Peak areas for ions that contained the specific lysine residue were recorded for all labeled and unlabeled ions. Percent modification for each lysine residue was reported as a percent of the total ion signal for the residue in question:

$$
\% \text { Mod }=\frac{\sum \text { Modified ion peak area }}{\sum \text { Modified peak area }+\sum \text { Unmodified peak area }}
$$

\subsubsection{Thioflavin $\mathrm{T}(\mathrm{ThT})$ assay.}

The assay was performed using a Molecular Devices M5 spectrophotometer (Molecular Devices, LLC, Sunnyvale, CA, USA). Stock ThT (Sigma Aldrich, St. Louis, MO, USA) was prepared in nanopure water at a concentration of $1.0 \mathrm{mg} / \mathrm{ml}$. Peptide labeling with NHSA was performed immediately after reconstitution, and immediately before analysis. $2.0 \mu \mathrm{L}$ of the stock ThT was spiked into the sample (100 $\mu 1$ total sample volume/well). Sample fluorescence was monitored using 440-nm excitation and 480-nm emission wavelengths. Samples were kept at $25^{\circ} \mathrm{C}$ for the duration of the assay. Emission data points were acquired every minute for $6 \mathrm{~h}$ for each NHSA concentration. Background fluorescence was obtained from a blank sample that contained no peptide, collected simultaneously with the analyte. Each peptide sample was run in triplicate and averaged. The average background signal was subtracted from each data point in the spectrum to obtain the actual fluorescence associated with $\beta$-sheet formation. Signal was normalized to the highest intensity value for each trial. 


\subsubsection{Purification of Glutathione S-transferase (GST)-htt-exon1 fusion proteins.}

GST-htt exon1 51Q fusion proteins were purified as described elsewhere [20, 60]. In short, expression of GST-htt fusion proteins was induced in Escherichia coli by isopropyl $\beta$-D-thiogalactoside for $4 \mathrm{~h}$ at $30^{\circ} \mathrm{C}$. The cells were lysed with $0.5 \mathrm{mg} / \mathrm{ml}$ of lysozyme. Liquid chromatography (LPLC, Bio-Rad) with a GST affinity column was used to purify the fusion proteins from the lysate. Relevant fractions to collect and purify were determined by gel electrophoresis, and purity of fusion proteins was consistently greater than 95\%. Prior to all experiments, solutions with all GST-htt exon1 51Q fusion proteins were high spin centrifuged at $20000 \mathrm{x}$ g for $30 \mathrm{~min}$ at $4^{\circ} \mathrm{C}$ to remove pre-existing aggregates. To initiate aggregation, the cleavage of the GST moiety was performed by the addition of Factor Xa protease (Promega, Madison, WI) for $1 \mathrm{~h}$ in ice. Experiments were carried out in PBS buffer.

\subsubsection{Acetylation of htt exon1 51Q.}

Htt was acetylated using selective covalent label, sulfo- $N$-hydroxysuccinimide acetate (NHSA) [61]. Stock solutions of NHSA were prepared in water to make $1.71 \mathrm{mg} / \mathrm{ml}$ solution. The labeling of htt was carried out in NHSA/htt molar ratios to achieve $1 \mathrm{x}, 2 \mathrm{x}$ and $3 \mathrm{x}$ NHSA labeling for $1 \mathrm{~min}$, and the reaction was quenched by adding $10 \mathrm{mM}$ Tris. The labeling was confirmed by MS. After acetylation, factor Xa protease was added to each protein sample and allowed to sit in ice for $1 \mathrm{~h}$ prior to bilayer experiments. 


\subsubsection{Protein samples for ex situ AFM imaging.}

The GST proteins (htt 51Q, $+1 \mathrm{x}$ NHSA, $+2 \mathrm{x}$ NHSA, $+3 \mathrm{x}$ NHSA) at $20 \mu \mathrm{M}$ in Eppendorf tubes were incubated at $37^{\circ} \mathrm{C}$ and $1400 \mathrm{rpm}$ for the duration of the experiment. At $0,1,3,5,8,24 \mathrm{~h}$ after cleavage of GST with factor Xa protease, aliquots $(5 \mu \mathrm{l})$ from each incubation sample were spotted onto freshly cleaved mica (Ted Pella Inc., Redding, CA), for 30seconds, washed with $200 \mu 1$ of ultra-pure water and dried gently with a stream of air. There mica was placed on metal pucks and imaged with AFM.

\subsubsection{Preparation of lipid bilayers.}

The lyophilized total brain lipid extract (TBLE) (Avanti Polar Lipids, Alabaster, AL) was reconstituted in $10 \mathrm{mM}$ PBS ( $\mathrm{pH} 7.3$ ) with vortexing to a concentration of $1 \mathrm{mg} / \mathrm{ml}$. Using liquid nitrogen, the lipid sample went through five cycles of freeze-thaw to facilitate bilayer and multilayer lipid sheet formation [62]. The lipid samples were then sonicated for $20 \mathrm{~min}$ to facilitate vesicle formation. A $40 \mu \mathrm{L}$ aliquot of $0.5 \mathrm{mg} / \mathrm{ml}$ TBLE was added into AFM fluid cell. A $25 \mu \mathrm{m}$ × $25 \mu \mathrm{m}$ lipid bilayer formed on the mica surface via vesicle fusion. The formation of the bilayer was monitored by continuous AFM imaging. Once fully formed, the bilayer was washed twice with $15 \mu$ l of PBS to remove excess lipid vesicles.

\subsubsection{AFM imaging conditions.}

All AFM experiments were performed with a Nanoscope V Multimode AFM (Veeco,Santa Barbara,CA) equipped with a closed loop vertical engage J-scanner. For ex situ AFM experiments, all images were acquired with diving board shaped silicon-oxide 
cantilevers with a nominal spring constant of $40 \mathrm{~N} / \mathrm{m}$ and resonance frequency around $300 \mathrm{kHz}$. For in situ AFM experiments, a tapping mode fluid cell with an $\mathrm{O}$ ring was equipped with a rectangular shaped silicon nitride cantilevers (Vista probes) with a nominal spring constant of $0.1 \mathrm{~N} / \mathrm{m}$. The scan rates were set between $1-2 \mathrm{~Hz}$ at 512 pixels per line and drive frequencies of $5-6 \mathrm{kHz}$ were used with the set point of 0.70 .

\subsubsection{Imaging Processing.}

All images were analyzed using Matlab with the image processing toolbox (MathWorks, Natick, MA) [63]. Physical dimensions of aggregates were measured automatically by (i) importing the images into Matlab, (ii) using a flattening algorithm to flatten the images to correct for background curvature, (iii) creating a binary map of the surface to locate individual aggregates using a height threshold, (iv) locating discrete aggregates by applying pattern recognition algorithms to the binary maps, and (v) finally the physical features (height, length etc) of individual aggregates were automatically measured .

\subsubsection{Preparation of TBLE/PDA vesicles.}

The polydiacetylene (PDA) assay was prepared using protocols previously described [64-67]. Briefly, TBLE and diacetylene monomer 10, 12 tricosadiynoic acid (GFS Chemicals, Columbus, $\mathrm{OH}$ ) in 2:3 molar ratio were mixed in $3 \mathrm{ml}$ of solution of ethanol /chloroform $(1: 1)$ in a round bottomed flask. The solution was dried by rotavap leaving a thin dry film. This was followed by the addition of $14.8 \mathrm{ml}$ of $1 \mathrm{x}$ Tris buffered saline (heated to $70^{\circ} \mathrm{C}$ ), and sonicated on high for $5 \mathrm{~min}$ at $100 \mathrm{~W}$ using a sonic 
dismembrator (FischerSci). The suspension was stored at $4^{\circ} \mathrm{C}$ overnight, then polymerized by irradiation with 7 lumens (UV analyzer) at $254 \mathrm{~nm}$ for $10 \mathrm{~min}$ while stirring thoroughly at room temperature giving a blue TBLE/PDA chromatic solution. The solution was kept in a glass container, covered with foil prior to experiments.

\subsubsection{Preparation of PDA/htt sample solutions.}

The assay included PDA $+1 \mathrm{x}$ Tris (negative control), $\mathrm{PDA}+1 \mathrm{x}$ Tris $+\mathrm{NaOH}(\mathrm{pH}$ 12; positive control), and PDA mixed with each of the four sample solutions described previously (htt-exon1 (51Q), htt +1x NHSA, htt $+2 x$ NHSA, htt $+3 x$ NHSA). Each sample was loaded in a 96 well plate in triplicate to achieve a final protein concentration of $20 \mu \mathrm{M}$ in each well. The kinetic absorbance measurements was recorded for $16 \mathrm{~h}$ on infinite M1000 Pro plate reader (TECAN, Switzerland) at $22^{\circ} \mathrm{C}$ with $3 \mathrm{sec}$ shaking between reads .The plate reader was set to record both the blue $(640 \mathrm{~nm})$ and the red component $(500 \mathrm{~nm})$ of the spectrum for each sample. Colorimetric response $(\% \mathrm{CR})$ was used as a quantitative measurement of PDA/protein interaction.

\subsection{Results}

\subsubsection{Chemically-induced acetylation occurs at all lysines in $\mathrm{Nt}^{17}$ and inhibits aggregation.}

In htt-exon1 there are three lysine residues available for acetylation that are located in $\mathrm{Nt}^{17}$ (K6, K9, and $\left.\mathrm{K} 15\right)$. To determine their availability for acetylation, model htt peptides were exposed to sulfo-N-hydroxysuccinimide acetate (NHSA), an established 
agent for labeling primary amines in proteins $[68,69]$. The model htt peptide contained the $\mathrm{Nt}^{17}$ domain, 35 repeat glutamines, a 10-residue polyproline domain, and two $\mathrm{C}$ terminal lysines $\left(\mathrm{Nt}^{17}-\mathrm{Q}_{35}-\mathrm{P}_{10}-\mathrm{KK}\right)$ to aid solubility. $\mathrm{Nt}^{17}-\mathrm{Q}_{35}-\mathrm{P}_{10}-\mathrm{KK}$ was used in place of the GST-fusion construct to reduce spectral complexity and eliminate ions that correspond to missed GST-htt cleavages. In cases where MS/MS data was insufficient or not available, the exact monoisotopic mass of a labeled ion was used for identification of an ion of interest. If this ion were produced from a peptide of a missed GST cleavage product, then this method would lead to a false-positive for a labeling event, skewing the results. As there are three lysines in $\mathrm{Nt}^{17}$, labeling was carried out in molar ratios of $1 \mathrm{x}$, $2 \mathrm{x}$, and $3 \mathrm{x}$ NHSA to htt, with quenching of the labeling reaction after 1 min by addition of $10 \mathrm{mM}$ Tris. Acetylated lysines were verified using chymotrypsin and MS to measure the relative amount of labeled and unlabeled fragments containing single lysine residues (Fig. 1a), and ThT assays were run to determine the impact of acetylation on $\mathrm{Nt}^{17}-\mathrm{Q}_{35^{-}}$ $\mathrm{P}_{10}$-KK aggregation (Fig. 1b). Chymotrypsin was selected over other serine proteases (such as trypsin) as its enzymatic activity is unaffected by substitution of the lysine primary amine. Overall, K9 was the most readily labeled, followed by K6 and K15. At 1x NHSA, all three lysines in $\mathrm{Nt}^{17}$ were labeled to some extent, but no more than $30 \%$. With 2x NHSA, K9 was acetylated to a significantly larger extent (69\%) than the other two lysines. At 3x NHSA, K9 acetylation increased to over $80 \%$, and over $60 \%$ of K6 was labeled. Acetylation of K15 increased with larger doses of NHSA, but it was never over $30 \%$ acetylated. The two flanking lysines at the C-terminus were not appreciably acetylated under any dose of NHSA. As acetylation increased, 


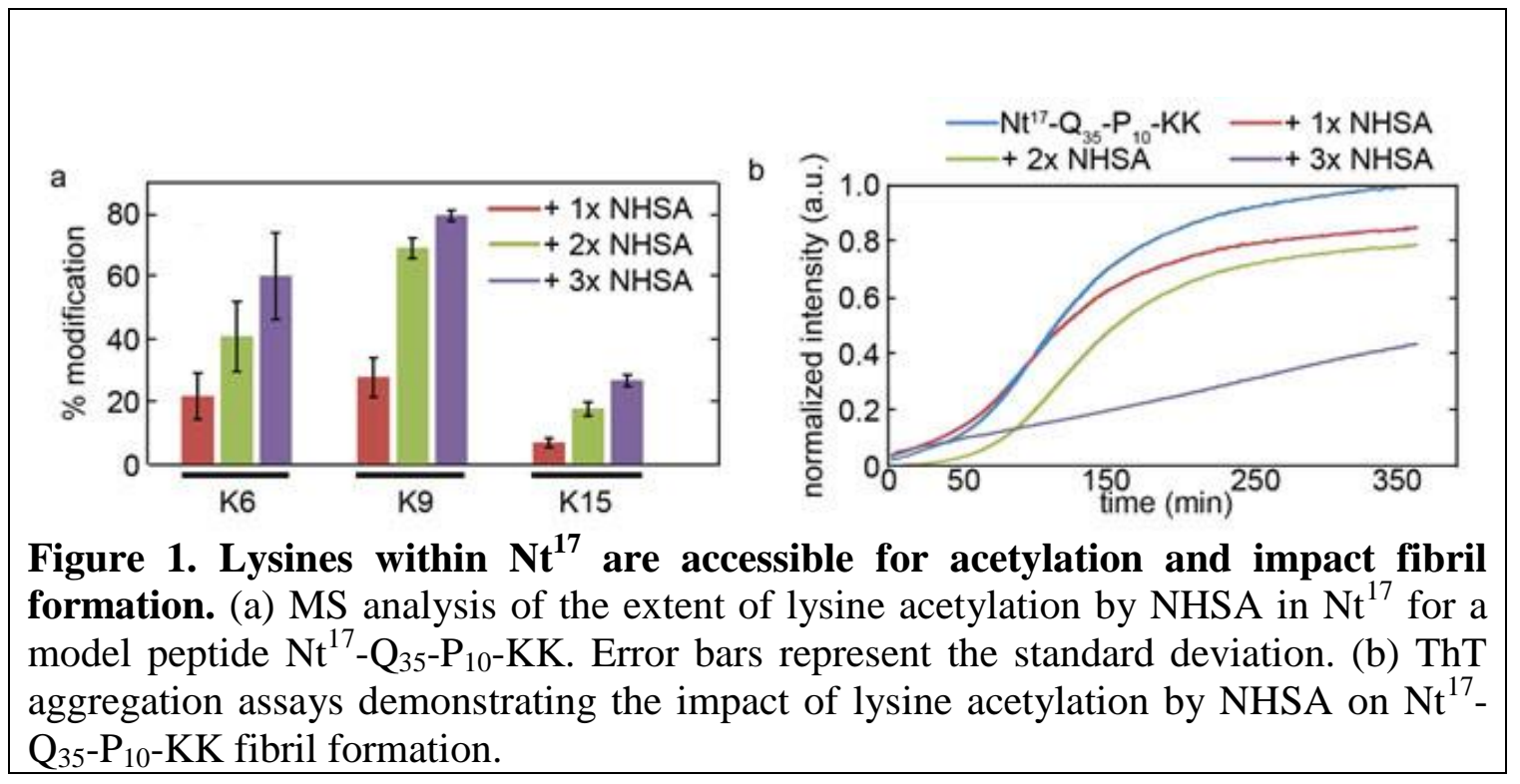

aggregation was inhibited in a dose dependent manner. At 1xNHSA, there was a slightly slower rate of aggregation (Fig. 1). 2x NHSA increased the lag phase with an overall reduced rate of aggregation. At 3x NHSA, aggregation was significantly decreased. This inhibition of aggregation suggests that K6 and K9 may play important roles in aggregation as reduced aggregation was observed once these residues were appreciably labeled.

\subsubsection{Acetylation of $\mathrm{Nt}^{17}$ inhibits htt-exon1 fibril formation.}

To determine the impact of acetylation on full length htt-exon1 aggregation, a mutant htt fragment, that expresses exon1 with 51Q (htt-exon1(51Q)), was exposed to different amounts of NHSA and the aggregation tracked. Htt-exon1(51Q) was purified from Escherichia coli as a soluble fusion with glutathione S-transferase (GST) [60]. The GST moiety was cleaved with a site-specific protease (Factor Xa), releasing the httexon1(51Q) fragment and initiating aggregation [70]. Htt-exon1(51Q) was exposed to NHSA in molar ratios of $0: 1,1: 1,2: 1$, or $3: 1$ in a similar fashion as $\mathrm{Nt}^{17}-\mathrm{Q}_{35}-\mathrm{P}_{10}-\mathrm{KK}$. To 


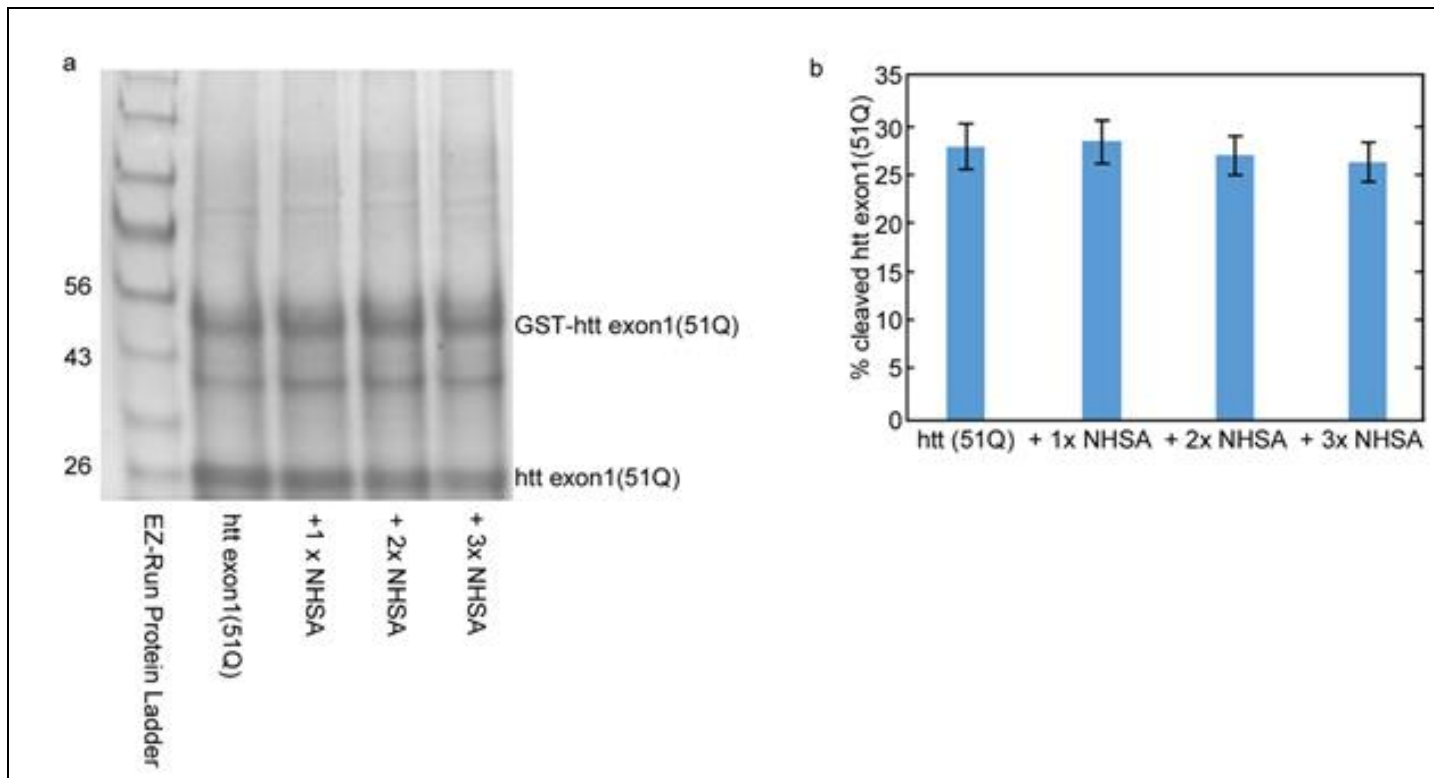

Figure 2. Addition of NHSA to GST-htt-exon1 (51Q) does not inhibit cleavage of the GST tag by Factor Xa. (a) SDS-PAGE demonstrating the efficiency of cleaving the GST tag of the fusion protein that had been acetylated with varying amounts of NHSA. (b) Densitometry analysis was used to determine the percent of cleaved httexon1 (51Q) as a function of total protein in the presence of varying amounts of NHSA.

verify that addition of the NHSA would not interfere with the removal of the GST tag, fusion protein that had been exposed to the different molar ratios of NHSA were incubated with Factor Xa on ice for one hour and analyzed using SDS-PAGE (Fig. 2). Densitometry analysis of the resulting gels demonstrated that there was no appreciable difference in the efficiency of cleaving the GST tag upon addition of NHSA.

Incubation experiments were performed with freshly prepared $20 \mu \mathrm{M}$ solutions of htt-exon1(51Q) that had been acetylated by NHSA at the different molar ratios. Aliquots were removed from the solutions after 1, 3, 5, 8 and $24 \mathrm{~h}$ of incubation for AFM analysis (Fig. 3). In an effort to quantify the effect of acetylation on htt aggregation, AFM images from all incubations were analyzed by counting the number of oligomers or fibrils per $\mu \mathrm{m}^{2}$ at each time point (Fig. 3b-c). For this analysis, aggregates were defined as objects in 


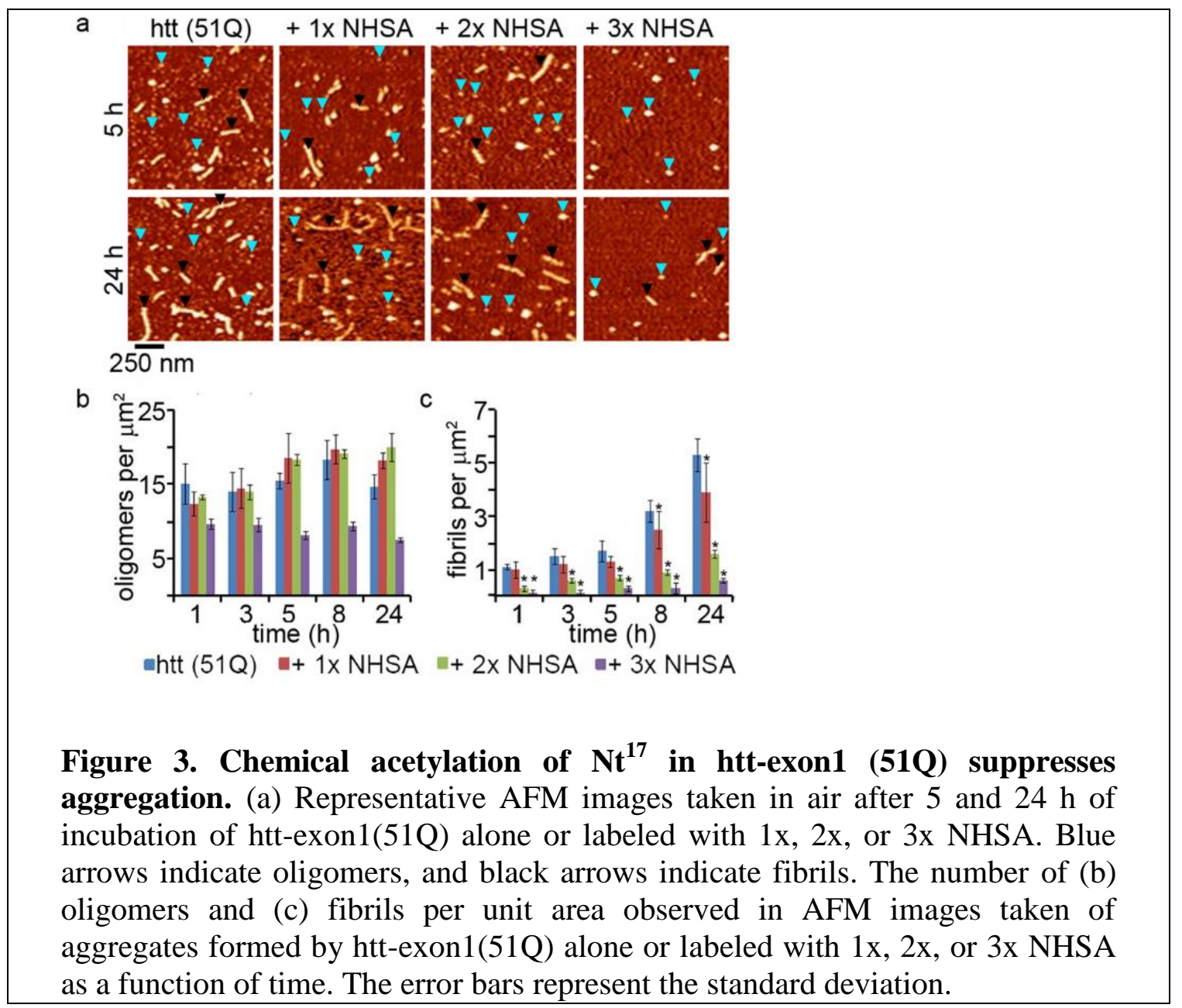

the image taller than $2 \mathrm{~nm}$. Fibrils were distinguished from oligomers by a length-towidth (aspect) ratio filter. Fibrils had an aspect ratio $>3$; oligomers had an aspect ratio $<3$ [63]. Htt-exon1(51Q) incubated without any NHSA quickly formed a variety of oligomers and fibrils (Fig. 3). The number of oligomers per unit area fluctuated from 1522 without any significant changes over $24 \mathrm{~h}$. The number of fibrils, however, steadily increased over $24 \mathrm{~h}$. At $1 \mathrm{~h}$, only a small number of fibrils were present, and these increased in number and grew from several hundred nanometers to $>1 \mu \mathrm{m}$ in length at later time points. When htt-exon1(51Q) was labeled with $1 \mathrm{x}$ NHSA, there was no significant difference in the number of oligomers compared to non-acetylated htt- 
exon1(51Q); however, after $8 \mathrm{~h}$, there was a significant decrease in the number of fibrils observed compared to the control (no acetylation). Increasing the NHSA treatment to $2 \mathrm{x}$ again resulted in no significant change in the number of oligomers observed, but there was a clear inhibition of fibril formation as early as after $1 \mathrm{~h}$ of incubation. Treatment with 3x NHSA resulted in a significant decrease in the number of oligomers and fibrils as a function of time. Overall, increasing the ratio of NHSA to htt decreased the number of fibrils that formed in a dose dependent manner, but only at 3x NHSA were the number of oligomers significantly affected.

Next, we compared the morphology of aggregates formed under the different acetylating conditions (Fig. 4). As the lateral dimensions contain appreciable contribution from the finite size and shape of the AFM probe, comparisons between the heights of aggregates are presented. Fibrils of htt-exon1(51Q) with increasing acetylation tended to be shorter, presumably due to a decreased time for fibril expansion due to a longer lag phase. However, the height along the contour of these fibrils was indistinguishable $(\sim 5-$ 6nm, Fig. 4a), suggesting a similar fibril structure. The size distribution of oligomers observed varied greatly with increasing acetylation (Fig. 4b). Without any NHSA, the distribution of oligomer size remained relatively tight with a slight increase in the mode height from 5-6 $\mathrm{nm}$ to 6-7 $\mathrm{nm}$ after $24 \mathrm{~h}$. The oligomer size distribution was similar up to $5 \mathrm{~h}$ of incubation when $1 \mathrm{x}$ NHSA was added, but at 8 and $24 \mathrm{~h}$ a pronounced population of larger oligomers $(\sim 9-11 \mathrm{~nm})$ began to form. This second, larger population of oligomers became more pronounced at earlier time points with increasing amounts of NHSA. With 3x NHSA, the larger population of oligomers eventually predominated the 


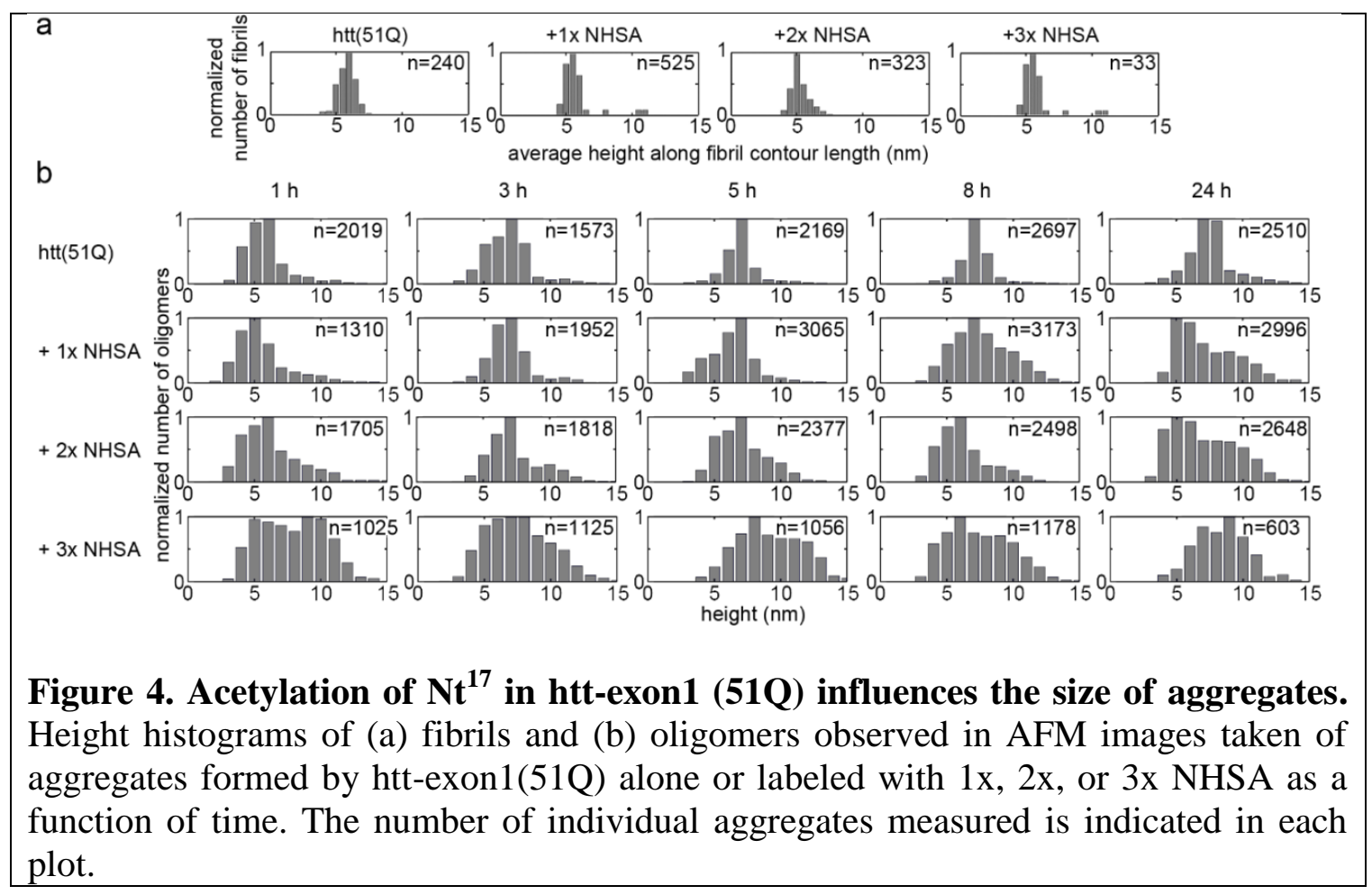

size distribution. The increased size of the oligomers formed with 3x NHSA may explain the significant reduction in the total number of oligomers as more peptide was incorporated into fewer aggregates.

\subsection{3 $\mathrm{Nt}^{17}$ Acetylation inhibits htt/lipid interactions.}

To determine if acetylation of $\mathrm{Nt}^{17}$ would alter the interaction of htt-exon 1 with lipid membranes, a total brain lipid extract (TBLE)/polydiacetylene (PDA) vesicle lipid binding assay was performed (Fig. 5a). This colorimetric assay uses mixed vesicles comprised of phospholipids and polymerized PDA. A rapid, visible color change from blue to red is induced by direct interaction between peptides and the phospholipid within the vesicles. The colorimetric response can be used to temporally track peptide- 


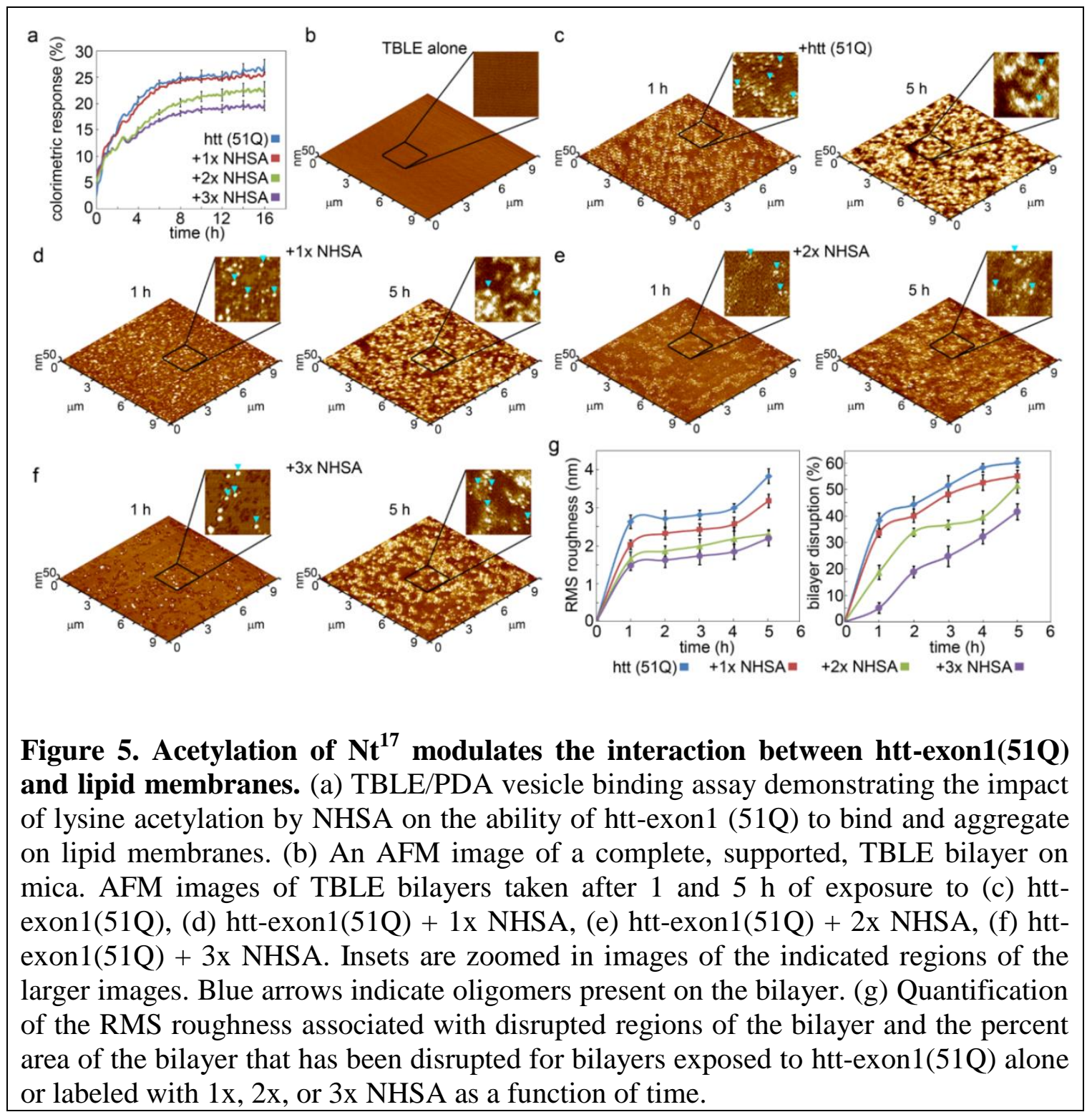

membrane interactions and PDA-based vesicles have been used to study a variety of biological processes at membrane surfaces, including the binding of amyloid proteins $[65,67,71,72]$. A quantitative value representative of the blue-to-red color transition is obtained by determining the \% colorimetric response (\%CR), defined as $[67,72]$ 


$$
\% \mathrm{CR}=\left[\left(\mathrm{PB}_{0}-\mathrm{PB}_{\mathrm{I}}\right) / \mathrm{PB}_{0}\right] \times 100
$$

where PB is a red/blue ratio of absorbance (A) defined as $A_{\text {blue }} /\left(A_{\text {blue }}+A_{\text {red }}\right)$. $A_{\text {blue }}$ is the absorbance at the "blue" component in the UV-vis spectrum $(\approx 640 \mathrm{~nm}) ; \mathrm{A}_{\text {red }}$ is absorbance at the "red" component $(\approx 500 \mathrm{~nm}), \mathrm{PB}_{0}$ is the red/blue ratio of the control sample (before induction of color change), and $\mathrm{PB}_{\mathrm{I}}$ is the value obtained for the vesicle solution after addition of peptides.

The TBLE/PDA vesicles were exposed to $20 \mu \mathrm{M}$ htt-exon1(51Q) or httexon1(51Q) that had been acetylated by NHSA (1x, 2x, or 3x), and \%CR was measured for 16 hours (Fig. 5a). Upon exposure to htt-exon1(51Q), there was an initial steady increase in $\% \mathrm{CR}$ as TBLE/PDA vesicles peptide bound and aggregated on the vesicle. Eventually, \%CR leveled off to a quasi-steady state value. There was not a significant change in the \%CR comparing exposures of unaltered htt-exon1(51Q) to that which had been exposed to 1x NHSA. After $\sim 14 \mathrm{~h}$, there was a small decrease in the $\% \mathrm{CR}$ between these two samples that was significant. However, as the amount of NHSA was increased to $2 \mathrm{x}$ or $3 \mathrm{x}$, there was a clear dose dependent reduction in the $\% \mathrm{CR}$ observed, indicating that acetylation reduced the interaction between htt-exon1 and the lipid membrane. Although, there is still an appreciable interaction of htt-exon1(51Q) with the lipid vesicles under all conditions.

To further investigate the impact of acetylation on the interaction of htt-exon1 with lipid bilayers, supported model lipid membranes through fusion of TBLE vesicles were prepared (Fig. 5b). Supported lipid bilayers preserve many important properties (i.e., lateral fluidity) of their free membrane counterparts [73, 74], and the interaction of 


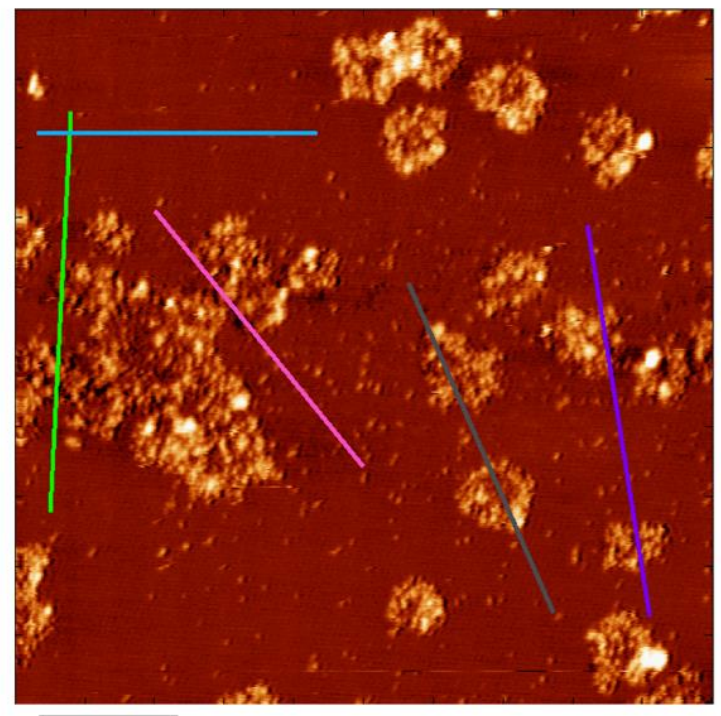

$1 \mu \mathrm{m}$

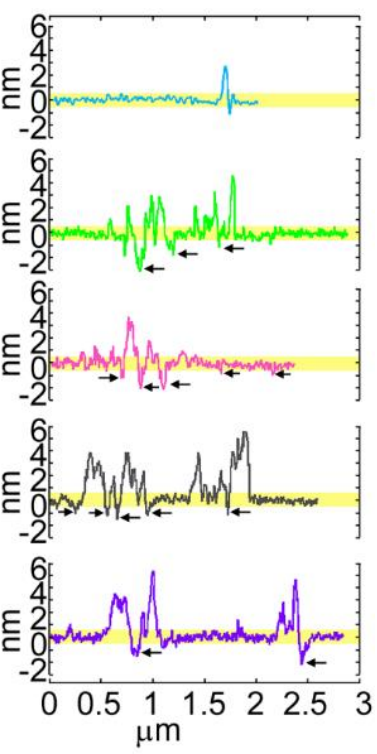

Figure 6. Exposure to htt-exon1(51Q) disrupts TBLE bilayers morphologically. A representative AFM image of a TBLE lipid bilayer exposed to htt-exon1(51Q) is presented. The colored lines in the image correspond to the profiles of the same color. The yellow shading on each profile represents the maximum roughness of an unperturbed TBLE bilayer. Arrows point to regions of the profiles that represent areas where partial holes or gaps have appeared in the bilayer.

amyloid-forming proteins with these bilayers can be continuously tracked using in solution AFM. Smooth bilayers (as determined by AFM) were exposed to unmodified htt-exon1(51Q) proteins or htt-exon1(51Q) that had been acetylated with varying amounts of NHSA $(1 \mathrm{x}, 2 \mathrm{x}$, or 3x NHSA). To cleave the GST moiety, Factor Xa was incubated with the GST- exon 1 fusion protein on ice for 1 hour prior to exposure to the supported bilayer. The resulting htt-exon 1 preparations predominately contain monomers and small oligomers $[4,63]$. Upon the initial exposure of the TBLE bilayer to $20 \mu \mathrm{M}$ freshly prepared htt-exon1(51Q), there was noticeable deposition of globular protein aggregates on the bilayer and regions of perturbed bilayer morphology (evidenced by an increase in surface roughness), consistent with previous reports (Fig. 5c) [49, 75]. The 
globular aggregates were stable on the bilayer surface as they were identifiable in consecutive images. The extent (or area) of bilayer perturbed by htt-exon1(51Q) increased with time. While most of the increased roughness appeared as the accumulation of protein above the initial bilayer surface, height profiles across these perturbed regions demonstrates that gaps and cavities within the bilayer also form (Fig. 6), verifying that bilayer structure was disrupted. This is consistent with previous reports that demonstrated by calcein dye leakage assays [19] and mechanical changes in bilayers [23, 49, 75] that htt-exon 1 can damage membranes. When TBLE bilayers were exposed to $20 \mu \mathrm{M}$ aliquots of htt-exon1(51Q) that had been chemically acetylated, similar morphological changes and globular aggregates compared with those associated with non-modified htt were observed within the bilayer (Fig. 5 d-f). However, the extent of bilayer perturbation appeared to be reduced as a function of increasing exposure to NHSA. Interestingly, fibril aggregates of any of the modified and nonmodified htt-exon1(51Q) proteins were not observed on the bilayer surface.

In an effort to quantify the extent of interaction between the TBLE bilayer and the different acetylated or non-acetylated htt-exon1(51Q) proteins, analysis of the AFM images was performed. First, the root mean squared (RMS) roughness of the perturbed regions of the bilayer was measured (Fig. 5g). A freshly formed TBLE bilayer has a RMS surface roughness of $0.3 \pm 0.05 \mathrm{~nm}$. To prevent error associated with the varying extent of perturbation of bilayer structure, RMS roughness measurements of areas that had been destabilized by modified or non-modified htt-exon1(51Q) were restricted only to portions of the images containing altered morphology. This allows for direct comparison of the extent of altered bilayer morphology contained within the perturbed regions. Within the 
first hour of exposure to the different acetylated or non-acetylated htt-exon1(51Q) constructs, regions of the bilayer that were significantly rougher $(\mathrm{p}<0.01)$ than unperturbed bilayer were observed; however, the roughness decreased with increasing acetylation. The roughness associated with perturbed bilayer regions remained relatively constant for $4 \mathrm{~h}$; however, there was a pronounced increase in roughness after $5 \mathrm{~h}$ of exposure to non-acetylated htt-exon1(51Q) or htt-exon1(51Q) with 1x NHSA.

As the previous analysis was confined just to regions of the bilayer that exhibited increased roughness, we next wanted to determine the extent of bilayer perturbation as a function of protein acetylation. To accomplish this, the percentage of the surface that displayed any increased roughness was determined (Fig. 5g). While the portion of the bilayer perturbed by acetylated or non-acetylated htt-exon1(51Q) steadily increased with time, the percent of the bilayer that was morphologically altered decreased as a function of increased protein acetylation. The trend corresponded well with the results from the first $5 \mathrm{~h}$ of the TBLE/PDA lipid binding assays. Furthermore, it cannot be determined if these globular aggregates are comprised of solely peptide or if they contain lipid components. The mode height of the oligomers observed on the bilayer was 2-3 nm for all experimental conditions and all time-points; although, larger aggregates increased in population over $5 \mathrm{~h}$.

Due to all of the potential acetylation sites in htt-exon1 being contained within the $\mathrm{Nt}^{17}$ and the known lipid-binding properties of $\mathrm{Nt}^{17}$, acetylation may change the affinity of this domain for lipid membranes. To address this, a series of $\mathrm{Nt}^{17}$ peptides were made that contained lysine to glutamine mutations to mimic acetylation. In this series of peptides, there are single, double, and triple mutations. TBLE/PDA lipid binding assays 


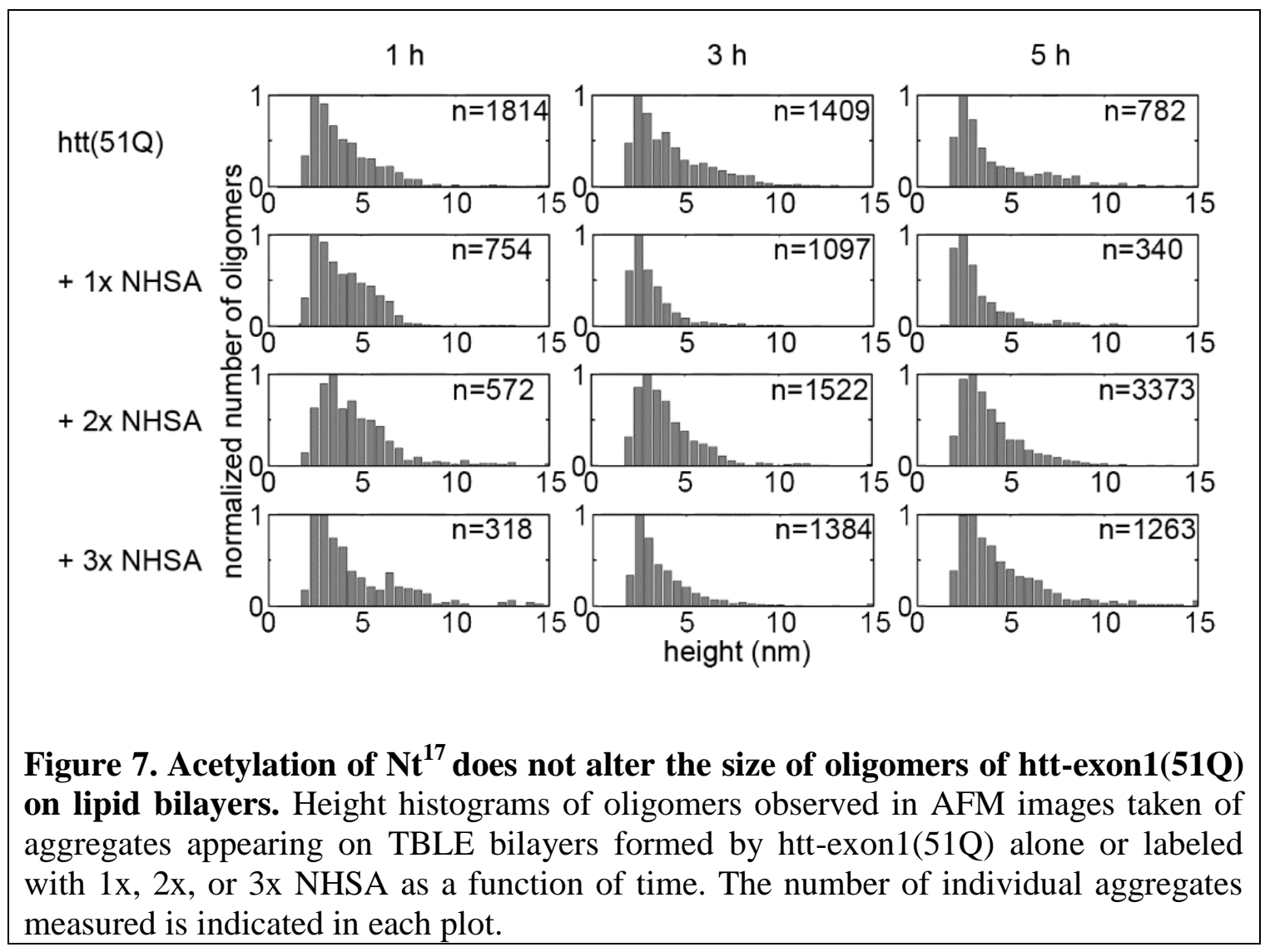

were performed to assess the relative affinity of these $\mathrm{Nt}^{17}$ peptides for lipid membranes (Fig. 8). By excluding the polyQ domain, the change in the \%CR in the PDA assay associated with aggregation can be removed, and the ability of these peptides to bind can be directly assessed. While htt-exon1 and synthetic peptides containing the polyQ domains have been shown to disrupt and aggregate on lipid membranes [19, 23, 49, 75], pure Nt17 peptides do not permeabilize lipid membranes [27]. All of the peptides containing a single mutation reduced the affinity of $\mathrm{Nt}^{17}$ for the vesicles, with the K9Q and K15Q mutants having a significantly lower affinity for the membrane compared with the K6Q mutant. This suggests that K9 and K15 may play a greater role in the interaction of $\mathrm{Nt}^{17}$ with lipid membranes. The double and triple mutants also reduced the affinity of $\mathrm{Nt}^{17}$ for the lipid membrane; however, only to a similar extent as the K6Q mutation alone. 

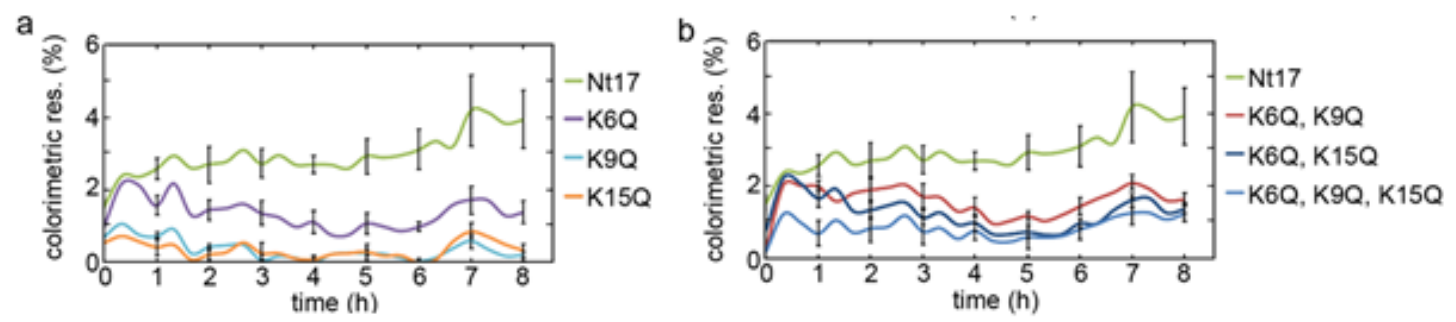

Figure 8. Acetylation-mimicking mutations reduce the affinity of $\mathrm{Nt}^{17}$ for lipid vesicles. TBLE/PDA vesicle binding assay performed with $\mathrm{Nt}^{17}$ peptides containing either (a) single mutation or (b) multiple mutations of lysine to glutamine to mimic acetylation.

As the overall \%CR is considerably smaller for the $\mathrm{Nt}^{17}$ peptides compared with httexon1(51Q) (Fig. 5a), aggregation on the vesicle surface appears to be the major contributor to the \%CR associated with exposure to the full htt-exon1.

\subsection{Discussion}

Although it is clear that polyQ expansion plays a key role in htt aggregation and toxicity, flanking sequences adjacent to the polyQ domain heavily influence aggregation, aggregate stability, important biochemical and functional properties of the protein, and ultimately htt's role in pathogenesis. In particular, the $\mathrm{Nt}^{17}$ domain of htt influences localization, aggregation, and degradation of htt in a cellular context $[21,22,43,57,76-$ 79]. Due to its ability to form an amphipathic $\alpha$-helix, $\mathrm{Nt}^{17}$ functions in regulating the binding of htt to a variety of membranous surfaces [21]. In this study, the impact of acetylation of Lysine residues within the $\mathrm{Nt}^{17}$ domain of htt on its aggregation and interactions with lipid membranes was investigated. Acetylation of $\mathrm{Nt}^{17}$ impeded the formation of htt fibrils and promoted the formation of larger, globular oligomers. 
Furthermore, acetylation lowered the affinity of $\mathrm{Nt}^{17}$ for lipid membranes, reducing membrane damage associated with exposure to htt. These include altered hydrogen bonding (both intrapeptide and peptide/phospholipid) and electrostatic interactions. As aberrant htt/lipid interactions have been implicated in a variety of toxic mechanisms, understanding how acetylation of $\mathrm{Nt}^{17}$ impact htt aggregation and its interaction with membranous surfaces could lead to therapeutic intervention with the potential implications to HD. For example, as acetylation in Nt17 is reduced in htt containing expanded polyQ domains in comparison with wild-type htt [80] and our data indicates that acetylation reduces the affinity of htt for lipid membranes, acetylating Nt17 may have a protective effect against htt/lipid interactions.

Computational studies performed in the lab of Dr. Silvina Matysiak at the University of Maryland suggest that the reduced affinity of $\mathrm{Nt}^{17}$ for lipid membranes caused by acetylation may be due to a number of factors associated with removal of the positive charge on lysine. The conformational dynamics of individual $\mathrm{Nt}^{17}$ domains with differing acetylated lysines in the presence of DOPC lipid bilayers were characterized via a computational approach using atomistic molecular dynamics. The aim of these simulations was to examine early events in the surface binding of $\mathrm{Nt}^{17}$ domain. Along with the wild type $\mathrm{Nt}^{17}$ sequence, three acetylation states were studied: $\mathrm{Nt}^{17}$ with $\mathrm{K} 9$ acetylated $\left(\mathrm{Nt}^{17}\right.$-ACK9); $\mathrm{Nt}^{17}$ with $\mathrm{K} 6$ and $\mathrm{K} 9$ acetylated $\left(\mathrm{Nt}^{17}\right.$-ACK6/9); $\mathrm{Nt}^{17}$ with $\mathrm{K} 6$, $\mathrm{K}$, and $\mathrm{K} 15$ acetylated $\left(\mathrm{Nt}^{17}-\mathrm{ACK} 6 / 9 / 15\right)$. Simulations of each peptide interacting with DOPC bilayers were run for $200 \mathrm{~ns}$. With unmodified $\mathrm{Nt}^{17}$, phenylalanine17 (F17) tended to insert into the membrane first, and this occurred within the first $50 \mathrm{~ns}$ of the simulation. Once F17 inserted into the membrane, hydrogen bonds with the membrane 
were then formed by nearby K15 and serine16 (S16). For the peptides containing various amounts of acetylation, F17 sometimes associated with the membrane (replicate 1 for $\mathrm{Nt}^{17}$-ACK9 and replicate 1 and 2 for $\mathrm{Nt}^{17}$-ACK6/9/15); however, this association tended to be transient compared to the non-acetylated peptide. With acetylation, interaction between the peptides and membrane would sometimes be initiated by residues 1-3, and this interaction was accompanied by hydrogen bond formation between threonine3 (T3) and the membrane. The stability of this interaction mediated by residues 1-3 was variable, as sometimes the peptide would remain bound to the membrane and other times the peptide would detach. Due to the transient nature observed in simulations of the interaction of different $\mathrm{Nt}^{17}$ peptides and the DOPC bilayer, the energetics of these interactions was determined. Attractive electrostatic interactions of significant magnitude (-300 to $-600+\mathrm{kJ} / \mathrm{mol})$ were often observed, but these were usually insufficient to cause permanent peptide association with the membrane. For permanent peptide association with the membrane, the magnitude of van der Waals interactions typically needed to exceed the magnitude of electrostatic interactions between the peptide and membrane. The largest contribution to van der Waals interactions between the peptide and membrane comes from hydrophobic residues.

$\mathrm{Nt}^{17}$ directly influences the abnormal aggregation of mutant htt. Specifically, $\mathrm{Nt}^{17}$ has been implicated in the initial stages of htt-exon1 aggregate formation [21, 26, 30, 36, 81]. As such, a variety of aggregation mechanisms have been proposed that are mediated by $\mathrm{Nt}^{17}$, with most supporting $\mathrm{Nt}^{17}$ self-association playing a key role in the formation of small, $\alpha$-helix rich oligomers $[25,28,82]$, suggesting that $\mathrm{Nt}^{17}$ promotes fibril formation through an oligomer mediated pathway [36, 51, 83]. With oligomers being widely 
considered as important toxic species in $\mathrm{HD}, \mathrm{Nt}^{17}$ directed oligomerization could play a vital role in htt-related toxicity. Indeed, self-association of $\mathrm{Nt}^{17}$ represents the ratedetermining step in the formation of htt-exon1 oligomers in cellular models [24, 84]. Here, it was demonstrated that acetylation of $\mathrm{Nt}^{17}$ slows htt aggregation and promotes the formation of larger oligomeric species. There are several plausible mechanisms that could explain this observation. One scenario is that acetylation promotes the formation of distinct htt oligomers that are off pathway to fibril formation. Alternatively, it is possible that acetylation stabilizes the $\alpha$-helix rich structure of the oligomers, delaying the transition to fibrils structures and providing time for the oligomers to increase in size by accumulating more monomers into the aggregate [24, 26, 85-92]. Other PTMs within $\mathrm{Nt}^{17}$ have already been shown to alter htt aggregation. For example, there are three phosphorylatable sites in $\mathrm{Nt}^{17}$ : $\mathrm{T} 3, \mathrm{~S} 13$, and $\mathrm{S} 16$, and several studies have found that htt phosphorylation is associated with reduced levels of mutant htt toxicity $[39,57,77,78$, 93-95]. Phosphorylation mimicking mutations of T3 enhanced htt aggregation in cultured cells and reduced lethality and neurodegeneration in Drosophila models of HD [57]. Phosphorylation-mimicking mutations of S13 and S16 significantly slowed htt aggregation and reduced fibril stability; however, fibril structure was not significantly changed [26, 77]. Interestingly, phosphorylation can regulate other PTMs, such as acetylation, and serve as a mechanism to target proteins for degradation [96]. Phosphorylation of S13 has been shown to influence acetylation at K9 [78]. It was also demonstrate here, that chemically acetylating lysines within $\mathrm{Nt}^{17}$ also can slow aggregation into fibrils without significantly altering the fibril structure. This finding further support the notion that $\mathrm{Nt}^{17}$ plays an important role in htt aggregation and, more 
specifically, that targeting the charged lysines within $\mathrm{Nt}^{17}$ may be a viable strategy for modulating aggregation for therapeutic purposes.

Full-length htt is a multi-domain protein that has been implicated with numerous, distinct cellular functions. With regards to the potential specificity of $\mathrm{Nt}^{17}$ for membranes of different composition, many membrane-associated functions are attributable to htt, such as cellular adhesion [97], motility [98, 99], cholesterol and energy homeostasis [100], molecular scaffolding for coordination of membrane and cytoskeletal communication [47], and facilitating microtubule-dependent vesicle transport [48]. Acetylation of $\mathrm{Nt}^{17}$ may play a role in regulating these activities. The trafficking of httexon1 between membranes associated with the ER, autophagic vacuoles, mitochondria, and Golgi appear to be facilitated by $\mathrm{Nt}^{17}[21,22]$. Trafficking of htt to specific subcellular compartments, for example the nucleus [101, 102], can have a strong influence in htt-related toxicity. The $\mathrm{Nt}^{17}$ domain can form an amphipathic $\alpha$-helix (AH) $[26,35,36]$, and number of biophysical functions are associated with AHs [103], including the ability to detect and bind highly curved lipid membranes [104-106]. As AHs tend to bend membranes weakly, their interaction with lipids can be easily regulated. With the large number of potential sites for post-translational modification, including acetylation, contained within $\mathrm{Nt}^{17}$, regulation of htt/lipid interactions via $\mathrm{Nt}^{17}$ may play a role in the trafficking of htt within the cellular environment. As such, the altered affinity upon acetylation of $\mathrm{Nt}^{17}$ for lipid membranes could play a role in trafficking htt within cells, with potential implications for both htt's normal function and toxicity. Such a scenario has been demonstrated for phosphorylation of $\mathrm{Nt}^{17}$, which can affect the nuclear export of htt $[21,107]$. It was also shown that acetylation of K9 plays a critical role clearance of htt 
via the proteasome and lysosome [78]. As a result, the acetylation of $\mathrm{Nt}^{17}$ and its modulating effect on membrane affinity may play a role in a variety of normal functions of htt. [27, 108, 109]

In a theoretical $\mathrm{AH}$, lysines 6 and 15 would be positioned at the interface between the hydrophilic and hydrophobic faces. It would appear that residues located in this interface may play a critical role in the ability of $\mathrm{Nt}^{17}$ to bind membranes. Simulations of htt peptide fragments associated with lipid membranes indicate that K6 and K15 form many hydrogen bond with bilayer components [34]. All atom simulations demonstrated that K6 and K15 often form salt-bridges with phospholipids; however, K9 was predominately involved in intramolecular salt-bridges with glutamic acid residues within $\mathrm{Nt}^{17}$ [50], suggesting that perturbing these residues would likely impact $\mathrm{Nt}^{17}$, s ability to bind membranes. A combination of experiments and simulations have indicated that the structure and orientation of $\mathrm{Nt}^{17}$ on membranes is mainly influenced by the sequestration of nonpolar residues into the hydrophobic core of a membrane; however, membrane composition influences the affinity of $\mathrm{Nt}^{17}$ for phospholipid bilayers via specific electrostatic interactions $[34,50]$. Collectively, this suggests a potential role for charged lysines in $\mathrm{Nt}^{17}$ selectively targeting membranes of specific phospholipid compositions, and removing their charge via acetylation could represent a mechanism to regulate this specificity. In this regard, mutating charged residues in $\mathrm{Nt}^{17}$, including the lysines, to alanines alters the localization of huntingtin within cells [21]. Furthermore, acetylation of a lysine (K444) beyond htt-exon1 has been implicated in autophagic removal of mutant htt [110]. Acetylation within $\mathrm{Nt}^{17}$ may play a similar role by regulating htt's interaction with lipid membranes [111]. 
Beyond acetylation, SUMOylation and ubiquitination can also occur at lysine residues within $\mathrm{Nt}^{17}$ and may also play a role in regulating the interaction of htt with membranes. SUMOylation is implicated to play a role in HD pathogenesis in both cell and animal models $[43,55]$. Specifically, SUMOylation at K6 and K9 reduce aggregation in cultured cells and exacerbate toxicity in Drosophila HD models [43]. Ubiquitination competes for the same lysine residues as SUMOylation, but it is associated with reducing mutant htt toxicity by tagging it for degradation by the ubiquitin proteasome system (UPS) [112]. As these other PTMs, are known to be involved in htt translocation within cells [21, 113]. Interestingly, mutating the lysine residues in $\mathrm{Nt}^{17}$ to arginine reduces cytotoxicity of Drosophila models of HD [43]. This reduction in cytotoxicity was attributed to the lack of lysine residues available for SUMOylation. Acetylation of lysine residues is a known mechanism to regulate the SUMOylation of proteins [114]. Furthermore, proteomic mapping by MS verified that K9 is appreciably acetylated in mammalian cell lysates [44]. The role of K9 acetylation has not yet been fully evaluated; however, acetylation of $\mathrm{Nt}^{17}$ may function in to regulate SUMOylation, modulating toxicity associated with SUMOylation of htt [110].

\subsection{Conclusion}

As the function, localization, and aggregation of htt have all been linked to $\mathrm{Nt}^{17}$, there is a need to identify at a mechanistic level how $\mathrm{Nt}^{17}$ influences the formation of toxic aggregates and its interaction with membranes. Here, it was demonstrated that lysine residues play an important role in both aggregation and membrane binding, and this role can be affected by removal of lysine's positive charge via acetylation. As 
acetylation in $\mathrm{Nt}^{17}$ is known to occur naturally [44, 110], the biophysical impact of this PTM could be important in htt's normal function, as well as have a modulating effect on toxicity related to mutant htt. Such knowledge is important because it could lead to better defined therapeutic targets (in addition to the polyQ tract) and strategies to treat HD. From the ongoing discussion, the importance of htt aggregation in HD has long been well articulated, and lipid bilayers have been shown to influence the aggregation of a variety of N-terminal htt fragments However, specific lipid components, such as cholesterol [115], sphingomyelin and gangliosides (GM1) can modulate htt aggregation on lipid bilayers as well.

\subsection{References}

1. Group, T.H.S.D.C.R., A Novel Gene Containing a Trinucleotide Repeat That Is Expanded and Unstable on Huntington's Disease Chromosomes Cell, 1993. 72: p. 971-983.

2. Snell, R.G., et al., Relationship between trinucleotide repeat expansion and phenotypic variation in huntingtons-disease. Nature Genetics, 1993. 4(4): p. 393397.

3. Tobin, A.J. and E.R. Signer, Huntington's disease: the challenge for cell biologists. Trends in Cell Biology, 2000. 10(12): p. 531-536.

4. Legleiter, J., et al., Mutant Huntingtin Fragments Form Oligomers in a Polyglutamine Length-dependent Manner in Vitro and in Vivo. Journal of Biological Chemistry, 2010. 285(19): p. 14777-14790. 
5. Vonsattel, J.P., et al., Neuropathological classification of huntingtons-disease. Journal of Neuropathology and Experimental Neurology, 1985. 44(6): p. 559-577.

6. Poirier, M.A., et al., Huntingtin spheroids and protofibrils as precursors in polyglutamine fibrilization. Journal of Biological Chemistry, 2002. 277(43): p. $41032-41037$.

7. Kim, Y.J., et al., Caspase 3-cleaved N-terminal fragments of wild-type and mutant huntingtin are present in normal and Huntington's disease brains, associate with membranes, and undergo calpain-dependent proteolysis. Proceedings of the National Academy of Sciences of the United States of America, 2001. 98(22): p. 12784-12789.

8. Cooper, J.K., et al., Truncated N-Terminal Fragments of Huntingtin with Expanded Glutamine Repeats form Nuclear and Cytoplasmic Aggregates in Cell Culture. Human Molecular Genetics, 1998. 7(5): p. 783-790.

9. Hoffner, G., M.-L. Island, and P. Djian, Purification of neuronal inclusions of patients with Huntington's disease reveals a broad range of $\mathrm{N}$-terminal fragments of expanded huntingtin and insoluble polymers. Journal of Neurochemistry, 2005. 95(1): p. 125-136.

10. Davies, S.W., et al., Formation of neuronal intranuclear inclusions underlies the neurological dysfunction in mice transgenic for the HD mutation. Cell, 1997. 90(3): p. 537-548.

11. Landles, C., et al., Proteolysis of Mutant Huntingtin Produces an Exon 1 Fragment That Accumulates as an Aggregated Protein in Neuronal Nuclei in Huntington Disease. J. Biol. Chem., 2010. 285(12): p. 8808-8823. 
12. Sathasivam, K., et al., Aberrant splicing of HTT generates the pathogenic exon 1 protein in Huntington disease. Proceedings of the National Academy of Sciences, 2013. 110(6): p. 2366-2370.

13. DiFiglia, M., et al., Aggregation of huntingtin in neuronal intranuclear inclusions and dystrophic neurites in brain. Science, 1997. 277(5334): p. 1990-1993.

14. Gutekunst, C.A., et al., Identification and localization of huntingtin in brain and human lymphoblastoid cell lines with anti-fusion protein antibodies. Proc Natl Acad Sci U S A, 1995. 92(19): p. 8710-4.

15. Gutekunst, C.A., et al., Nuclear and neuropil aggregates in Huntington's disease: Relationship to neuropathology. Journal of Neuroscience, 1999. 19(7): p. 25222534.

16. Kodali, R., et al., A beta(1-40) Forms Five Distinct Amyloid Structures whose beta-Sheet Contents and Fibril Stabilities Are Correlated. Journal of Molecular Biology, 2010. 401(3): p. 503-517.

17. Kegel, K.B., et al., Huntingtin expression stimulates endosomal-lysosomal activity, endosome tubulation, and autophagy. Journal of Neuroscience, 2000. 20(19): p. $7268-7278$.

18. Qin, Z.H., et al., Huntingtin bodies sequester vesicle-associated proteins by a polyproline-dependent interaction. Journal of Neuroscience, 2004. 24(1): p. 269281.

19. Suopanki, J., et al., Interaction of huntingtin fragments with brain membranes clues to early dysfunction in Huntington's disease. Journal of Neurochemistry, 2006. 96(3): p. 870-884. 
20. Valencia, A., et al., Mutant Huntingtin and Glycogen Synthase Kinase 3-beta Accumulate in Neuronal Lipid Rafts of a Presymptomatic Knock-In Mouse Model of Huntington's Disease. Journal of Neuroscience Research, 2010. 88(1): p. 179190.

21. Atwal, R.S., et al., Huntingtin has a membrane association signal that can modulate huntingtin aggregation, nuclear entry and toxicity. Human Molecular Genetics, 2007. 16(21): p. 2600-2615.

22. Rockabrand, E., et al., The first 17 amino acids of Huntingtin modulate its subcellular localization, aggregation and effects on calcium homeostasis. Human Molecular Genetics, 2007. 16(1): p. 61-77.

23. Burke, K.A., et al., The Interaction of Polyglutamine Peptides With Lipid Membranes is Regulated by Flanking Sequences Associated with Huntingtin. Journal of Biological Chemistry, 2013.

24. Jayaraman, M., et al., Slow Amyloid Nucleation via alpha-Helix-Rich Oligomeric Intermediates in Short Polyglutamine-Containing Huntingtin Fragments. Journal of Molecular Biology, 2012. 415(5): p. 881-899.

25. Thakur, A.K., et al., Polyglutamine disruption of the huntingtin exon $1 \mathrm{~N}$ terminus triggers a complex aggregation mechanism. Nature Structural \& Molecular Biology, 2009. 16(4): p. 380-389.

26. Mishra, R., et al., Inhibiting the Nucleation of Amyloid Structure in a Huntingtin Fragment by Targeting alpha-Helix-Rich Oligomeric Intermediates. Journal of Molecular Biology, 2012. 415(5): p. 900-917. 
27. Michalek, M., et al., Membrane Interactions of the Amphipathic Amino Terminus of Huntingtin. Biochemistry, 2013. 52(5): p. 847-858.

28. Williamson, T.E., et al., Modulation of Polyglutamine Conformations and Dimer Formation by the N-Terminus of Huntingtin. Journal of Molecular Biology, 2010. 396(5): p. 1295-1309.

29. Dlugosz, M. and J. Trylska, Secondary Structures of Native and Pathogenic Huntingtin N-Terminal Fragments. Journal of Physical Chemistry B, 2011. 115(40): p. 11597-11608.

30. Kelley, N.W., et al., The Predicted Structure of the Headpiece of the Huntingtin Protein and Its Implications on Huntingtin Aggregation. Journal of Molecular Biology, 2009. 388(5): p. 919-927.

31. Côté, S., G. Wei, and N. Mousseau, All-Atom Stability and Oligomerization Simulations of Polyglutamine Nanotubes with and without the 17-Amino-Acid NTerminal Fragment of the Huntingtin Protein. The Journal of Physical Chemistry B, 2012. 116(40): p. 12168-12179.

32. Côté, S., G. Wei, and N. Mousseau, Atomistic mechanisms of huntingtin Nterminal fragment insertion on a phospholipid bilayer revealed by molecular dynamics simulations. Proteins: Structure, Function, and Bioinformatics, 2014. 82(7): p. 1409-1427.

33. Lakhani, V.V., F. Ding, and N.V. Dokholyan, Polyglutamine Induced Misfolding of Huntingtin Exon1 is Modulated by the Flanking Sequences. Plos Computational Biology, 2010. 6(4): p. e1000772. 
34. Nagarajan, A., S. Jawahery, and S. Matysiak, The Effects of Flanking Sequences in the Interaction of Polyglutamine Peptides with a Membrane Bilayer. The Journal of Physical Chemistry B, 2013. 118(24): p. 6368-6379.

35. Sahoo, B., et al., Aggregation Behavior of Chemically Synthesized, Full-Length Huntingtin Exon1. Biochemistry, 2014. 53(24): p. 3897-3907.

36. Sivanandam, V.N., et al., The Aggregation-Enhancing Huntingtin N-Terminus Is Helical in Amyloid Fibrils. Journal of the American Chemical Society, 2011. 133(12): p. 4558-4566.

37. Michalek, M., Evgeniy S. Salnikov, and B. Bechinger, Structure and Topology of the Huntingtin 1-17 Membrane Anchor by a Combined Solution and Solid-State NMR Approach. Biophysical Journal, 2013. 105(3): p. 699-710.

38. Arndt, J.R., et al., Lysine residues in the N-terminal huntingtin amphipathic $\alpha$ helix play a key role in peptide aggregation. Journal of Mass Spectrometry, 2015. 50(1): p. 117-126.

39. Ehrnhoefer, D.E., L. Sutton, and M.R. Hayden, Small changes, big impact: posttranslational modifications and function of huntingtin in Huntington disease. Neuroscientist, 2011. 17(5): p. 475-92.

40. Wang, J., et al., Potential application of grape derived polyphenols in huntington's disease. Translational Neuroscience, 2010. 1(2): p. 95-100.

41. Young, F.B., et al., Putting proteins in their place: palmitoylation in Huntington disease and other neuropsychiatric diseases. Prog Neurobiol, 2012. 97(2): p. 22038. 
42. Pennuto, M., I. Palazzolo, and A. Poletti, Post-translational modifications of expanded polyglutamine proteins: impact on neurotoxicity. Human Molecular Genetics, 2009. 18(R1): p. R40-R47.

43. Steffan, J.S., et al., SUMO modification of Huntingtin and Huntington's disease pathology. Science, 2004. 304(5667): p. 100-104.

44. Cong, X., et al., Mass spectrometric identification of novel lysine acetylation sites in huntingtin. Mol Cell Proteomics, 2011. 10(10): p. M111 009829.

45. Kegel-Gleason, K.B., Huntingtin Interactions with Membrane Phospholipids: Strategic Targets for Therapeutic Intervention? Journal of Huntington's Disease, 2013. 2(3): p. 239-250.

46. Leoni, V. and C. Caccia, The impairment of cholesterol metabolism in Huntington disease. Biochimica et Biophysica Acta (BBA) - Molecular and Cell Biology of Lipids, 2015(0).

47. Kegel, K.B., et al., Huntingtin associates with acidic phospholipids at the plasma membrane. Journal of Biological Chemistry, 2005. 280(43): p. 36464-36473.

48. Caviston, J.P. and E.L.F. Holzbaur, Huntingtin as an essential integrator of intracellular vesicular trafficking. Trends in Cell Biology, 2009. 19(4): p. 147155.

49. Burke, K.A., et al., Huntingtin disrupts lipid bilayers in a polyQ-length dependent manner. Biochimica Et Biophysica Acta-Biomembranes, 2013. 1828(8): p. 19531961. 
50. Côté, S., et al., Probing the Huntingtin 1-17 Membrane Anchor on a Phospholipid Bilayer by Using All-Atom Simulations. Biophysical Journal, 2015. 108(5): p. $1187-1198$.

51. Arndt, J.R., M. Chaibva, and J. Legleiter, The emerging role of the first 17 amino acids of huntingtin in Huntington's disease, in Biomolecular Concepts2015. p. 33.

52. Dorval, V. and P.E. Fraser, SUMO on the road to neurodegeneration. Biochimica et Biophysica Acta (BBA)-Molecular Cell Research, 2007. 1773(6): p. 694-706.

53. Díaz-Hernández, M., et al., Inhibition of $26 S$ proteasome activity by huntingtin filaments but not inclusion bodies isolated from mouse and human brain. Journal of neurochemistry, 2006. 98(5): p. 1585-1596.

54. Borrell-Pages, M., et al., Huntington's disease: from huntingtin function and dysfunction to therapeutic strategies. Cellular and Molecular Life Sciences, 2006. 63(22): p. 2642-2660.

55. O'Rourke, J.G., et al., SUMO-2 and PIAS1 modulate insoluble mutant huntingtin protein accumulation. Cell Rep, 2013. 4(2): p. 362-75.

56. Hannoun, Z., et al., Post-translational modification by SUMO. Toxicology, 2010. 278(3): p. 288-93.

57. Aiken, C.T., et al., Phosphorylation of Threonine 3: Implications for huntingtin aggregation and neurotoxicity. Journal of Biological Chemistry, 2009. 284(43): p. 29427-29436.

58. Thakur, A.K., et al., Polyglutamine disruption of the huntingtin exon $1 \mathrm{~N}$ terminus triggers a complex aggregation mechanism. Nature Structural \& Molecular Biology, 2009. 16(4): p. 380-389. 
59. Chen, S.M. and R. Wetzel, Solubilization and disaggregation of polyglutamine peptides. Protein Science, 2001. 10(4): p. 887-891.

60. Muchowski, P.J., et al., Hsp70 and Hsp40 chaperones can inhibit self-assembly of polyglutamine proteins into amyloid-like fibrils. Proceedings of the National Academy of Sciences of the United States of America, 2000. 97(14): p. 78417846.

61. Mendoza, V.L., et al., Structural Insights into the Pre-Amyloid Tetramer of $\beta$-2Microglobulin from Covalent Labeling and Mass Spectrometry. Biochemistry, 2011. 50(31): p. 6711-6722.

62. Yip, C.M., et al., Cholesterol, a modulator of membrane-associated A betafibrillogenesis and neurotoxicity. Journal of Molecular Biology, 2001. 311(4): p. 723-734.

63. Burke, K.A., J. Godbey, and J. Legleiter, Assessing mutant huntingtin fragment and polyglutamine aggregation by atomic force microscopy. Methods, 2011. 53(3): p. 275-284.

64. Jelinek, R. and T. Sheynis, Amyloid - Membrane Interactions: Experimental Approaches and Techniques. Current Protein and Peptide Science, 2010. 11(5): p. 372-84.

65. Kolusheva, S., L. Boyer, and R. Jelinek, A colorimetric assay for rapid screening of antimicrobial peptides. Nat Biotech, 2000. 18(2): p. 225-227.

66. Porat, Y., et al., The Human Islet Amyloid Polypeptide Forms Transient Membrane-Active Prefibrillar Assembliest. Biochemistry, 2003. 42(37): p. 10971-10977. 
67. Sokolovski, M., et al., Membrane interactions and lipid binding of casein oligomers and early aggregates. Biochim Biophys Acta, 2008. 1778(10): p. 23419.

68. Markoutsa, S., et al., Sulfo-NHS-SS-biotin derivatization: A versatile tool for MALDI mass analysis of PTMs in lysine-rich proteins. Proteomics, 2014. 14(6): p. 659-667.

69. Mendoza, V.L., et al., Structural insights into the pre-amyloid tetramer of beta-2microglobulin from covalent labeling and mass spectrometry. Biochemistry, 2011. 50(31): p. 6711-22.

70. Scherzinger, E., et al., Self-assembly of polyglutamine-containing huntingtin fragments into amyloid-like fibrils: Implications for Huntington's disease pathology. Proceedings of the National Academy of Sciences of the United States of America, 1999. 96(8): p. 4604-4609.

71. Yates, E.A., et al., Specific domains of $A \beta$ facilitate aggregation on and association with lipid bilayers. Journal of Molecular Biology, (0).

72. Zheng, F., Z. Wu, and Y. Chen, A quantitative method for the measurement of membrane affinity by polydiacetylene-based colorimetric assay. Anal Biochem, 2012. 420(2): p. 171-6.

73. Groves, J.T., N. Ulman, and S.G. Boxer, Micropatterning fluid lipid bilayers on solid supports. Science, 1997. 275(5300): p. 651-653.

74. Jass, J., T. Tjarnhage, and G. Puu, From liposomes to supported, planar bilayer structures on hydrophilic and hydrophobic surfaces: An atomic force microscopy study. Biophysical Journal, 2000. 79(6): p. 3153-3163. 
75. Burke, K.A., E.A. Yates, and J. Legleiter, Amyloid-Forming Proteins Alter the Local Mechanical Properties of Lipid Membranes. Biochemistry, 2013. 52(5): p. 808-817.

76. Atwal, R.S., et al., Kinase inhibitors modulate huntingtin cell localization and toxicity. Nature Chemical Biology, 2011. 7(7): p. 453-460.

77. Gu, X., et al., Serines 13 and 16 Are Critical Determinants of Full-Length Human Mutant Huntingtin Induced Disease Pathogenesis in HD Mice. Neuron, 2009. 64(6): p. 828-840.

78. Thompson, L.M., et al., IKK phosphorylates Huntingtin and targets it for degradation by the proteasome and lysosome. Journal of Cell Biology, 2009. 187(7): p. 1083-1099.

79. Zheng, Z., et al., An N-terminal Nuclear Export Signal Regulates Trafficking and Aggregation of Huntingtin (Htt) Protein Exon 1. Journal of Biological Chemistry, 2013. 288(9): p. 6063-6071.

80. Cong, X., et al., Mass spectrometric identification of novel lysine acetylation sites in huntingtin. Molecular \& Cellular Proteomics, 2011. 10(10): p. M111. 009829.

81. Crick, S.L., et al., Unmasking the roles of $\mathrm{N}$ - and $\mathrm{C}$-terminal flanking sequences from exon 1 of huntingtin as modulators of polyglutamine aggregation. Proceedings of the National Academy of Sciences, 2013. 110(50): p. 2007520080.

82. Vitalis, A. and R.V. Pappu, Assessing the contribution of heterogeneous distributions of oligomers to aggregation mechanisms of polyglutamine peptides. Biophysical Chemistry, 2011. 159(1): p. 14-23. 
83. Bugg, C.W., et al., Structural features and domain organization of huntingtin fibrils. The Journal of biological chemistry, 2012. 287(38): p. 31739-46.

84. Olshina, M.A., et al., Tracking Mutant Huntingtin Aggregation Kinetics in Cells Reveals Three Major Populations That Include an Invariant Oligomer Pool. Journal of Biological Chemistry, 2010. 285(28): p. 21807-21816.

85. Kar, K., et al., Critical nucleus size for disease-related polyglutamine aggregation is repeat-length dependent. Nature Structural \& Molecular Biology, 2011. 18(3): p. 328-+.

86. Colby, D.W., et al., Potent inhibition of huntingtin and cytotoxicity by a disulfide bond-free single-domain intracellular antibody. Proceedings of the National Academy of Sciences of the United States of America, 2004. 101(51): p. 1761617621.

87. Colby, D.W., et al., Development of a human light chain variable domain (V-L) intracellular antibody specific for the amino terminus of huntingtin via yeast surface display. Journal of Molecular Biology, 2004. 342(3): p. 901-912.

88. Tam, S., et al., The chaperonin TRiC controls polyglutamine aggregation and toxicity through subunit-specific interactions. Nature cell biology, 2006. 8(10): p. 1155-1162.

89. Tam, S., et al., The chaperonin TRiC blocks a huntingtin sequence element that promotes the conformational switch to aggregation. Nature Structural \& Molecular Biology, 2009. 16(12): p. 1279-U98. 
90. McLear, J.A., et al., Combinational approach of intrabody with enhanced Hsp70 expression addresses multiple pathologies in a fly model of Huntington's disease. Faseb Journal, 2008. 22(6): p. 2003-2011.

91. Wolfgang, W.J., et al., Suppression of Huntington's disease pathology in Drosophila by human single-chain Fv antibodies. Proceedings of the National Academy of Sciences of the United States of America, 2005. 102(32): p. 1156311568.

92. De Genst, E., et al., Structure of a Single-Chain Fv Bound to the $17 \mathrm{~N}$-Terminal Residues of Huntingtin Provides Insights into Pathogenic Amyloid Formation and Suppression. Journal of Molecular Biology, 2015. 427(12): p. 2166-2178.

93. Warby, S.C., et al., Huntingtin phosphorylation on serine 421 is significantly reduced in the striatum and by polyglutamine expansion in vivo. Journal of Neurology Neurosurgery and Psychiatry, 2005. 76.

94. Humbert, S. and F. Saudou, Huntingtin phosphorylation and signaling pathways that regulate toxicity in Huntington's disease. Clinical Neuroscience Research, 2003. 3(3): p. 149-155.

95. Watkin, E.E., et al., Phosphorylation of Mutant Huntingtin at Serine 116 Modulates Neuronal Toxicity. Plos One, 2014. 9(2).

96. Hunter, T., The Age of Crosstalk: Phosphorylation, Ubiquitination, and Beyond. Molecular Cell, 2007. 28(5): p. 730-738.

97. Strehlow, A.N.T., J.Z. Li, and R.M. Myers, Wild-type huntingtin participates in protein trafficking between the Golgi and the extracellular space. Human Molecular Genetics, 2007. 16(4): p. 391-409. 
98. Myre, M.A., et al., Deficiency of Huntingtin Has Pleiotropic Effects in the Social Amoeba <italic>Dictyostelium discoideum</italic>. PLoS Genet, 2011. 7(4): p. e1002052.

99. Ritch, J.J., et al., Multiple phenotypes in Huntington disease mouse neural stem cells. Molecular and Cellular Neuroscience, 2012. 50(1): p. 70-81.

100. Jacobsen, J.C., et al., HD CAG-correlated gene expression changes support a simple dominant gain of function. Human Molecular Genetics, 2011. 20(14): p. 2846-2860.

101. Saudou, F., et al., Huntingtin acts in the nucleus to induce apoptosis but death does not correlate with the formation of intranuclear inclusions. Cell, 1998. 95(1): p. 55-66.

102. Schilling, G., et al., Nuclear-targeting of mutant huntingtin fragments produces Huntington's disease-like phenotypes in transgenic mice. Human Molecular Genetics, 2004. 13(15): p. 1599-1610.

103. Drin, G. and B. Antonny, Amphipathic helices and membrane curvature. FEBS Letters, 2010. 584(9): p. 1840-1847.

104. Brass, V., et al., An Amino-terminal Amphipathic $\alpha$-Helix Mediates Membrane Association of the Hepatitis C Virus Nonstructural Protein 5A. Journal of Biological Chemistry, 2002. 277(10): p. 8130-8139.

105. Georgieva, Elka R., et al., Tau Binds to Lipid Membrane Surfaces via Short Amphipathic Helices Located in Its Microtubule-Binding Repeats. Biophysical Journal, 2014. 107(6): p. 1441-1452. 
106. Cui, H., E. Lyman, and G.A. Voth, Mechanism of Membrane Curvature Sensing by Amphipathic Helix Containing Proteins. Biophysical Journal, 2011. 100(5): p. 1271-1279.

107. Maiuri, T., et al., The huntingtin N17 domain is a multifunctional CRM1 and Randependent nuclear and cilial export signal. Human Molecular Genetics, 2013. 22(7): p. 1383-1394.

108. Michalek, M., C. Aisenbrey, and B. Bechinger, Investigation of membrane penetration depth and interactions of the amino-terminal domain of huntingtin: refined analysis by tryptophan fluorescence measurement. European Biophysics Journal, 2014. 43(8-9): p. 347-360.

109. Chaibva, M., K.A. Burke, and J. Legleiter, Curvature Enhances Binding and Aggregation of Huntingtin at Lipid Membranes. Biochemistry, 2014. 53(14): p. 2355-2365.

110. Jeong, H., et al., Acetylation Targets Mutant Huntingtin to Autophagosomes for Degradation. Cell, 2009. 137(1): p. 60-72.

111. Thompson, L.M., et al., IKK phosphorylates Huntingtin and targets it for degradation by the proteasome and lysosome. Journal of Cell Biology, 2009. 187(7): p. 1083-1099.

112. Jana, N.R., et al., Co-chaperone CHIP associates with expanded polyglutamine protein and promotes their degradation by proteasomes. Journal of Biological Chemistry, 2005. 280(12): p. 11635-11640.

113. Xia, J.R., et al., Huntingtin contains a highly conserved nuclear export signal. Human Molecular Genetics, 2003. 12(12): p. 1393-1403. 
114. Ullmann, R., et al., An Acetylation Switch Regulates SUMO-Dependent Protein Interaction Networks. Molecular Cell, 2012. 46(6): p. 759-770.

115. Gao, X., et al., Cholesterol Modifies Huntingtin Binding to, Disruption of, and Aggregation on Lipid Membranes. Biochemistry, 2015. 


\section{Outlook: Other relevant post translational modifications and small molecules as potential modulators of huntingtin aggregation.}

The work presented in this dissertation was aimed at elucidating interactions of htt with lipid membranes from both a physical and biochemical perspective. It starts with development and validation of supported lipid bilayers with curvature, followed by investigating the impact of membrane curvature on synthetic truncated htt exon 1 mimicking peptides $\left(\mathrm{Nt}^{17} \mathrm{Q}_{35} \mathrm{P}_{10} \mathrm{KK}\right)$ on aggregation. This was followed by expounding the role of acetylation on huntingtin aggregation, both kinetically and morphologically in the presence of lipid membranes. The major goal of these studies was to understand physicochemical aspects and molecular mechanisms associated with aggregation in the presence of cellular and subcellular surfaces, particularly lipid membranes. The ultimate goal was to understand potential ways to prevent or slow down mutant huntingtin aggregation, and eventually leading to the development of therapies since there is no widely effective preventative or therapeutic treatments for HD. A broad range of research tools and spectrophotometric assays such ThT and PDA were employed in this study. However, owing to its robust ability to provide particularly useful insights related to neurodegenerative diseases, AFM was utilized as the primary tool in my studies. AFM has the unique ability to be operated in air (ex situ) and in solution (in situ), allowing direct probing of biological samples at solid-liquid interfaces, under nearly physiological conditions.

In future, it is important to investigate how the changing the composition of lipid bilayers by exogenously adding monosialotetrahexosylganglioside known as GM1,

5. Outlook: Other relevant post translational modifications and small molecules as potential modulators of huntingtin aggregation. 
influences htt binding and aggregation on lipid membranes. Several other factors that can potentially regulate the aggregation of htt at lipid need further investigation. One of such factors is the role of several other important post translational modifications (PTMs) on htt aggregation on lipid bilayers. Phosphorylation and SUMOylation are some of the modifications that have been implicated in HD pathogenesis; however the impact of these modifications on htt aggregation on lipid membranes has not yet been evaluated. Finally, apart from PTMs, there is increasing evidence that small molecules, heat shock proteins (HSPs) included, could counteract common pathological mechanisms that take place in neurodegenerative diseases such as Alzheimer's disease (AD), Parkinson's disease (PD) and HD. It is important to understand how such molecules would interfere with htt's potential to form toxic oligomeric assemblies on lipid membranes.

\subsection{Work in progress: Lipid components modulates huntingtin aggregation on lipid membranes}

The brain and membrane of neurons are enriched in different kinds of fats and/or lipids. GM1 which is a major component of lipid rafts is a notable example. In the brain, GM1 have a plethora of functions , ranging from cell signaling [1] , to neurotransmission [2], suggesting that even a small reduction in GM1 levels will likely have a huge physiological impact on HD neurons. In 2010, Sipione and coworkers from the University of Alberta, Canada, observed a reduction in the synthesis of GM1 in a variety of HD animal models as well as in fibroblasts in HD patients [3]. They further showed that neuronal cells with decreased GM1 are more susceptible to programmed cell death leading to neurodegeneration. What was even more intriguing about these studies is that

5. Outlook: Other relevant post translational modifications and small molecules as potential modulators of huntingtin aggregation. 
administration of GM1 by continuous infusion into YAC 128 mice, not only restores the GM1 levels but reverses cell apoptosis, leading to improved motor function and complete reversal of the impairment in YAC 128 mouse HD models [3, 4]. There could be several mechanisms to explain this restoration in motor function in symptomatic mice. GM1 induces serine S13 and S16 phosphorylation, which could explain the mechanism of this restoration [3-5]. However, since neurons are primarily composed of lipid membranes, another mechanism might lie in the role of lipid membranes in HD. Considering the importance of these finding, together with the role of GM1 in brain neurons, and how lipid membranes are important in HD pathogenesis, it is therefore of paramount importance to understand how exogenous added GM1 affects htt aggregation on lipid bilayers.

\subsubsection{Cholesterol and GM1 alters the extent of htt-exon1 binding to lipid vesicles as determined by TBLE/PDA binding assay.}

Prior to the GM1 experiment, the impact of added cholesterol on TBLE lipids was evaluated as preliminary work. To verify that altering the lipid composition of TBLE would modulate the interaction of htt-exon1 with lipids, a TBLE/PDA vesicle lipid binding assay performed. As described in chapter 4, PDA is a colorimetric assay in which mixed vesicles composed of lipids and polymerized chromatic PDA lipids exhibit rapid visible color changes from blue to red prompted by interactions between biomolecular species and the phospholipid components within the vesicles. The colorimetric response can be used to quantify protein-membrane interactions. The TBLE/PDA vesicles were

5. Outlook: Other relevant post translational modifications and small molecules as potential modulators of huntingtin aggregation. 


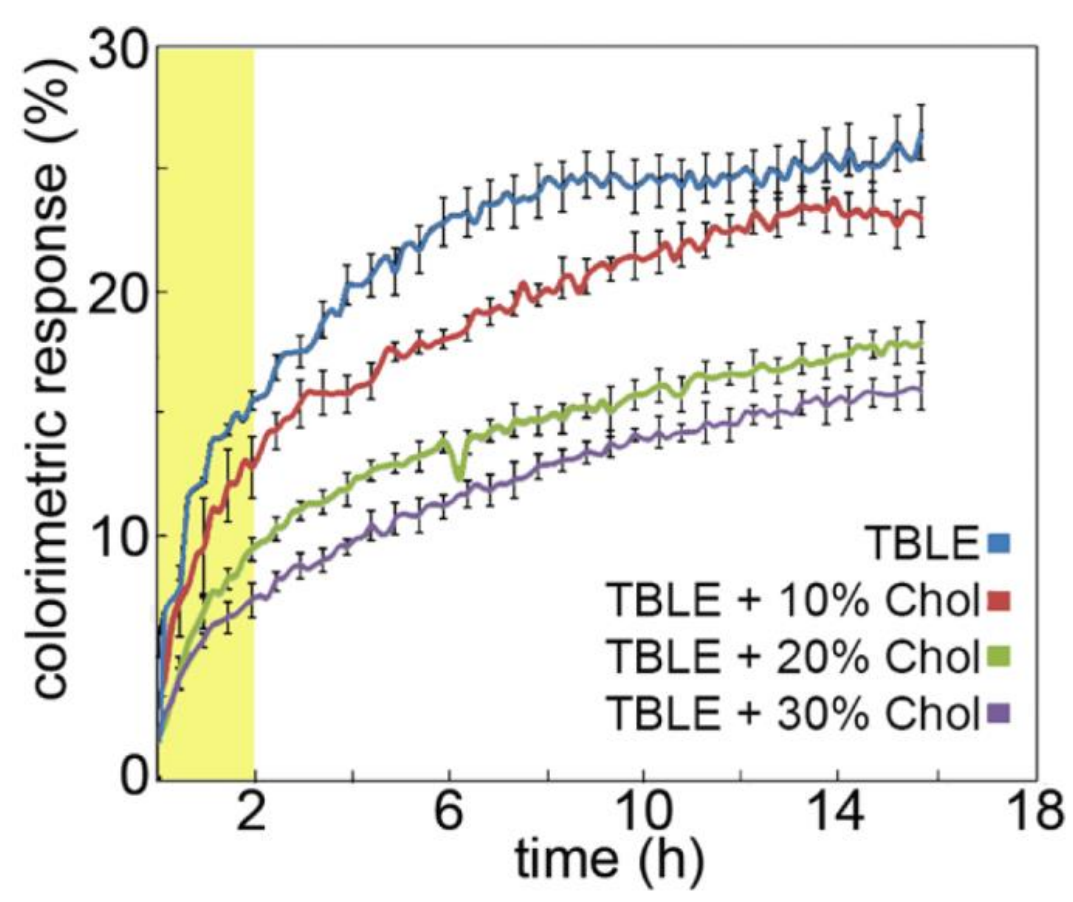

Figure 1. Percent colorimetric response (\%CR) of TBLE/PDA vesicles containing various amounts of cholesterol upon exposure to htt-exon1 (51Q) plotted as a function of time. Error bars indicate one standard deviation $(n=3)$. The highlighted area indicates the time frame accessible with AFM experiments

systematically enriched with $0 \%$ (pure TBLE), $10 \%, 20 \%$, or $30 \%$ cholesterol or $0 \%$ (pure TBLE), $5 \%, 10 \%$, or $15 \%$ GM1 by mass. These vesicles were exposed to htt exon1 protein and colorimetric response (\%CR) was measured for $16 \mathrm{~h}$.Characteristically, there is an initial steady increase in \%CR as TBLE/ PDA vesicles are exposed to htt-exon1. The \%CR tends to level off with time to a steady state. As the exogenous cholesterol or GM1 content was enhanced from $0 \%$ to $30 \%$ and $0 \%$ to $15 \%$ respectively, the binding of htt-exon1 (51Q) to the vesicles was reduced, highlight that lipid composition modulates htt aggregation on membranes (Fig.1 and Fig.2 respectively). The quasi-steady state $\% \mathrm{CR}$ is also significantly smaller in a cholesterol or GM1 dose-dependent manner,

5. Outlook: Other relevant post translational modifications and small molecules as potential modulators of huntingtin aggregation. 


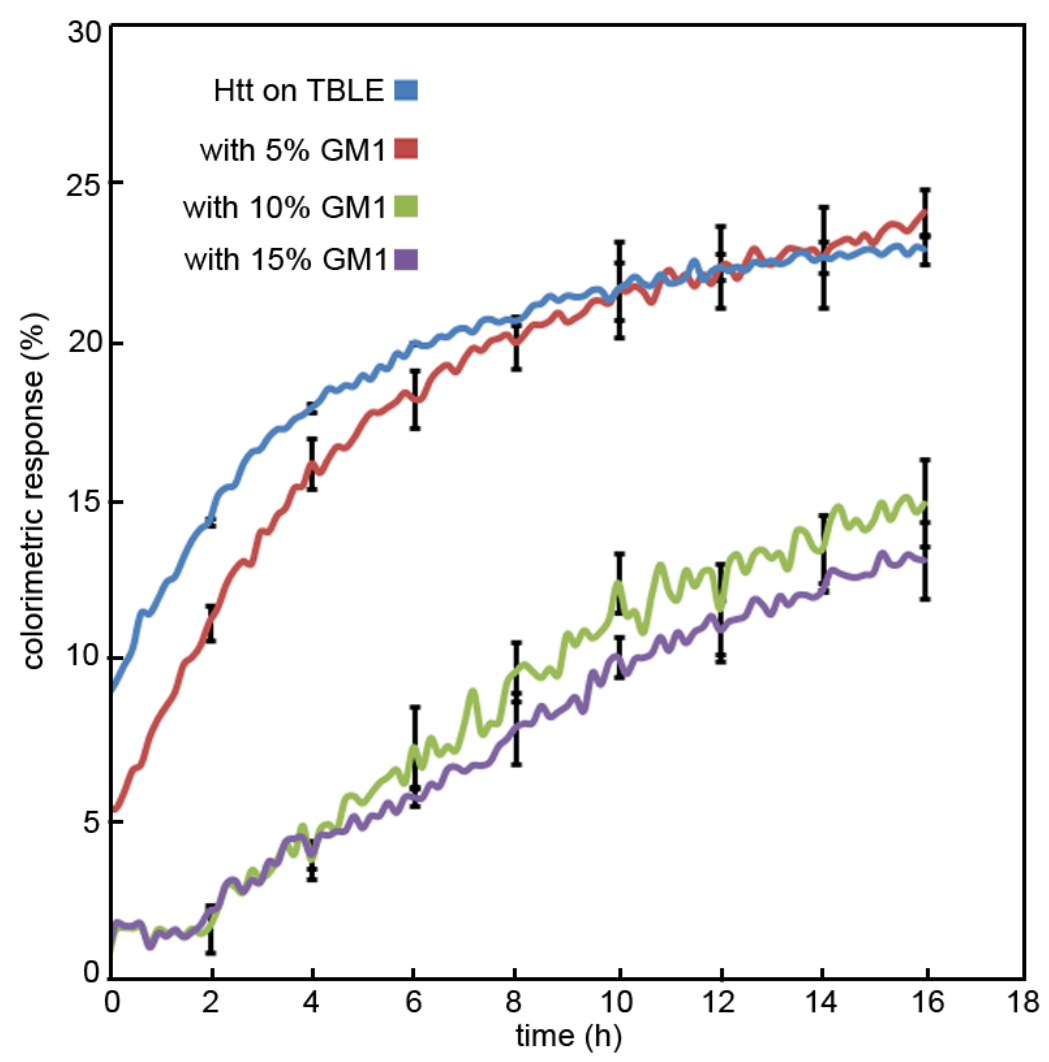

Figure 2. Percent colorimetric response (\%CR) of TBLE/PDA vesicles containing various amounts of GM1 upon exposure to htt-exon1(51Q) plotted as a function of time. Error bars indicate one standard deviation $(n=3)$.

indicating that cholesterol and GM1 reduces htt-exon1 (51Q) overall binding to and insertion into lipid membranes. The complete influence of cholesterol on the aggregation of htt on lipid membranes supported TBLE bilayers containing various amounts of cholesterol were further studied using AFM, and the findings have already been published.

For GM1 studies, as in cholesterol, further studies to explore the impact of GM1 on the aggregation of htt on supported TBLE bilayers containing various amounts of GM1 were conducted. Lipid bilayers were exposed to htt-exon1 and continuously imaged potential modulators of huntingtin aggregation. 


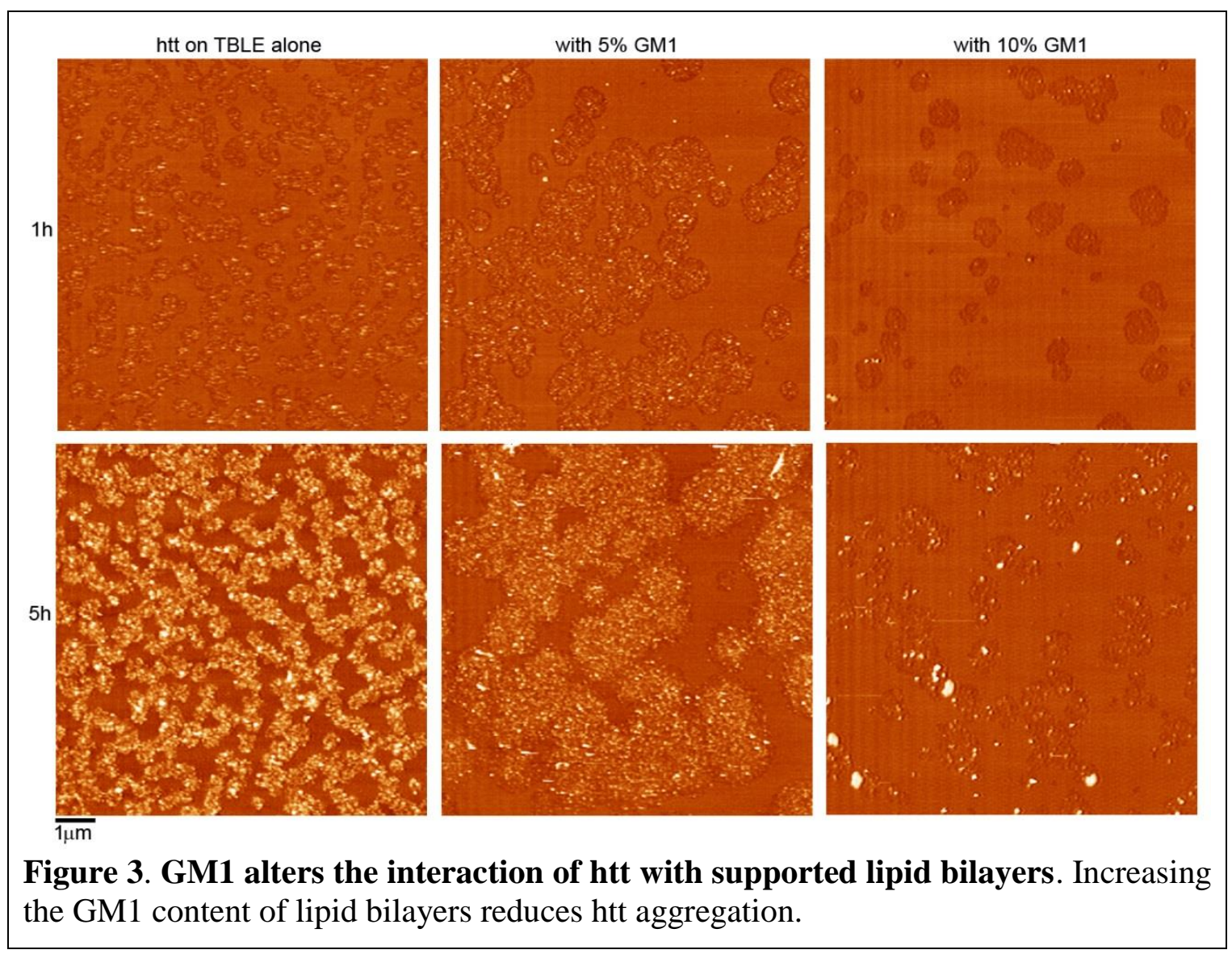

using in situ AFM, allowing for the direct tracking of protein aggregation on the bilayer. Bilayers comprised of pure TBLE, TBLE + 5\% GM1, and TBLE + 10\% GM1 were used. As shown in Fig.3, as GM1 is enriched, the bilayers slow down htt aggregation, suggesting that GM1 could one potential way to control aggregation of htt on lipid bilayers. This supports other studies, which reported that restoring GM1 levels in HD mice, prevents the striatal cells from apoptosis.

5. Outlook: Other relevant post translational modifications and small molecules as potential modulators of huntingtin aggregation. 


\subsection{Future studies:}

\subsubsection{Post translational modifications (PTMs) on huntingtin aggregation.}

There are other important PTMs on $\mathrm{Nt}^{17}$ that could be crucial modulators of htt aggregation and toxicity. The commonly studied ones are SUMOylation and phosphorylation [6-8]. SUMOylation is the enzymatic covalent attachment of SUMO (small ubiquitin-like modifier proteins) to the lysine residues of a target protein. SUMO are $11 \mathrm{kDa}$ proteins that are highly conserved in a variety of species and can be enzymatically reversibly attached to lysine residue of htt $[9,10]$. The SUMO conjugation pathway is a three step process involving the use of the heterodimer E1 activating enzyme (Ubc2/ Aos1), an E2 conjugating enzyme (Ubc9) and the E3 ligating enzymes $[11,12]$. The $\mathrm{Nt}^{17}$ domain of htt exon 1 contains three potential lysine SUMOylation sites, (K6, K9 and K15), and this domain also functions in lipid binding. The SUMOylation of lysines 6 and $9(\mathrm{~K} 6, \mathrm{~K} 9)$ in the drosophila HD model expressing htt was found to enhance neurodegeneration [13] ,while in cultured striatal neurons, SUMOylation stabilizes htt exon 1 and decreases its aggregation. It is evident that SUMOylation has been linked to HD pathogenesis [13-16], but the mechanistic details is often still poorly understood $[13,15]$, and the question still remains, what is the mechanism of pathology? Htt is found in association with brain lipids [17, 18] and membranes in cells [19], and PTMs are thought to regulate the lipid/htt interaction [20]. It has also been reported that various cellular components as well as lipids modulates htt aggregation and toxicity [21-23]. The studying of SUMOylation on htt's interaction with lipid membranes has not yet been explored. SUMOylation has been involved in the trafficking of htt, and this involves the interaction of htt with lipid vesicles. In subsequent

5. Outlook: Other relevant post translational modifications and small molecules as potential modulators of huntingtin aggregation. 
projects, it therefore important to investigate the impact of SUMOylation on htt aggregation at lipid interfaces in order to get mechanistic details of how SUMOylation influences htt aggregation and how SUMOylated htt interacts with lipid membranes with potential implications to HD.

Phosphorylation is another extensively studied PTM in which a phosphate group or a phosphate mimics is added to the threonine or serine residues of a protein. In context of htt exon 1, the $\mathrm{Nt}^{17}$ has three phosphorylation sites, threonine 3 (T3) and serines 13 and 16 (S13 and S16). It was shown that phosphomimetic mutation of S13 and S16 by aspartic acid substitution, resulted in decreased toxicity of mutant htt in different animal models of HD possibly by changing protein conformation and function [5, 24, 25]. Since surfaces, particularly lipid membranes, play important roles in neurodegenerative diseases, it is imperative to understand how htt phosphorylation modulates the protein aggregation at lipid interfaces.

\subsubsection{Targeting huntingtin $\mathrm{Nt}^{17}$ with small molecules}

The $\mathrm{Nt}^{17}$ domain inn htt exon 1 , both in free solution and in the presence of lipids, has been found to influence the formation of various aggregate species including the small, highly diffuse, and more toxic oligomers [26, 27]. One of the several ways to slow

down protein aggregation or change aggregation pathway is by blocking $\mathrm{Nt}^{17}$ or making itin unavailable for self-association [26, 28-30]. From previous studies, polyproline (polyP) PPII type helix can have a regulatory effect on aggregation $\mathrm{Nt}^{17}$ [31]. Collaboratively I carried out preliminary studies (unpublished) on how polyP peptides interact with $\mathrm{Nt}^{17}$ of htt exon 1 . Htt exon 1 was co-incubated with polpP peptides at

5. Outlook: Other relevant post translational modifications and small molecules as potential modulators of huntingtin aggregation. 


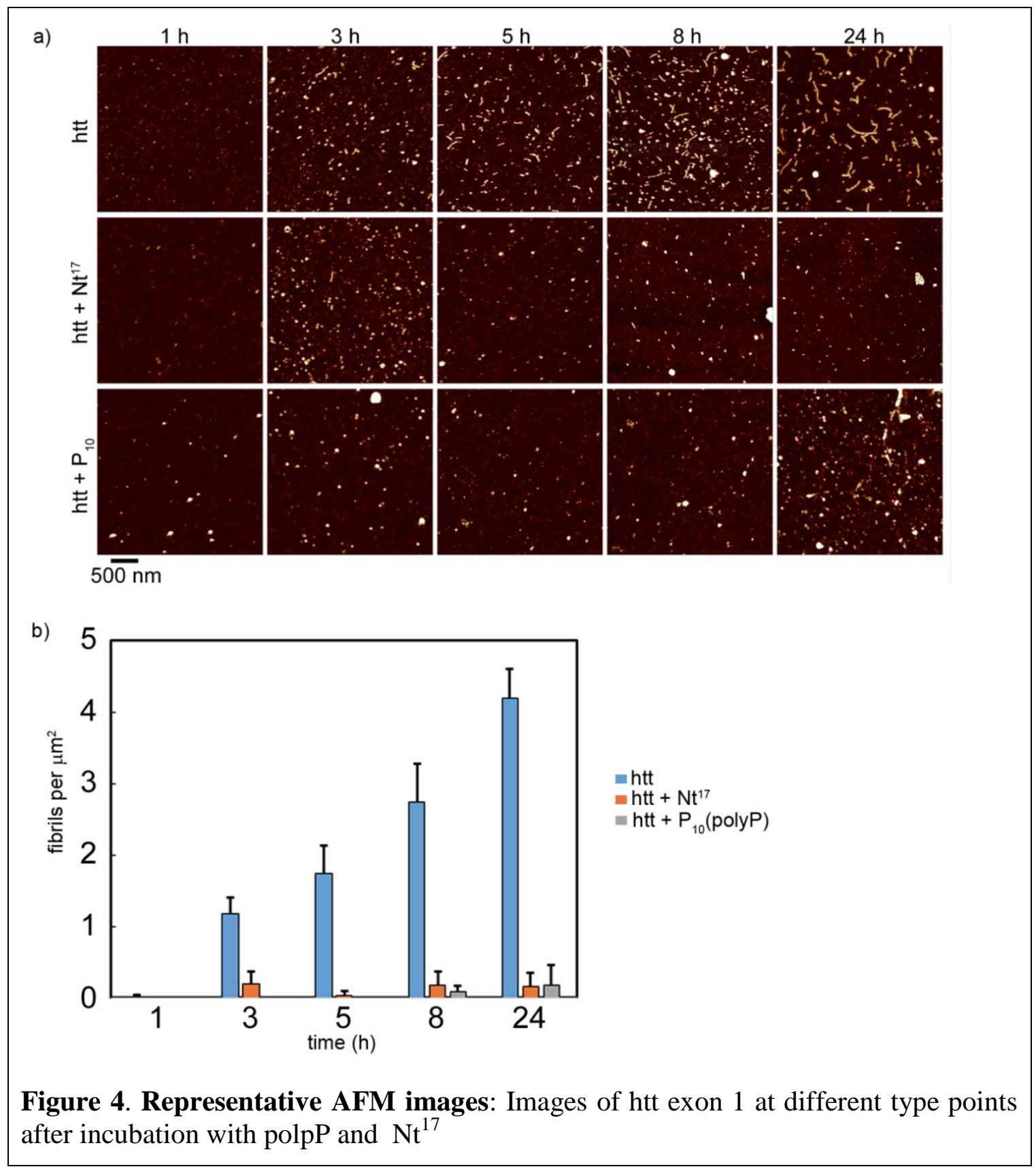

$37^{\circ} \mathrm{C}$ and incubated for 24 hours to allow aggregate formation. As shown Fig.4, htt exon

1 forms well defined fibrils in a time-dependent manner. After incubation with $\mathrm{Nt}^{17}$ and polyP, the fibril formation is greatly reduced. This is in agreement with previous

5. Outlook: Other relevant post translational modifications and small molecules as potential modulators of huntingtin aggregation. 
observations in which oligomers are observed when htt is incubated with polyP, suggesting that polyP peptide has inhibitory effect on amyloid formation [5]. This could be due to the polyP peptide interacting with the polyQ domain, stabilizing it and prevents the transition to $\beta$-sheet structure.

It has further been reported that under bulk conditions, several amyloid inhibitors prevent the formation of aggregate forming species in neurodegenerative [32]. It has also become evident that amyloidal species such as oligomers and fibrils exert their toxic effects at cellular membranes than in bulk solution. Studying how small molecules such heat shock proteins (Hsc70), EGCG, or curcumin interact with htt at the phospholipid interface could provide critical information on mechanisms by which such molecules inhibit amyloid formation associated with neurodegenerative. In a separate study, fatty acid metabolism and its compartmentalization appeared disordered in individuals with HD [33]. Fatty acids composition has also been implicated in the fluidity of lipid membranes and HD patients have altered membrane fluidity. The precise mechanism on how omega-3-fatty acids contribute to HD pathology especially on sub-cellular surfaces, is not well understood, and needs to be further attention.

\subsection{Concluding remarks and Perspectives}

Huntington's disease is a fatal, neurodegenerative disorder caused by an expansion of the polyQ tract in first exon 1 of htt. It is slightly more than two decades after the discovery of the IT15 gene, and several strides in HD have been yielding very promising results but no effective treatment has been identified to date, and much more remains to be understood about HD pathogenesis. The results presented in this

5. Outlook: Other relevant post translational modifications and small molecules as potential modulators of huntingtin aggregation. 
dissertation provide some invaluable insights into some of the physicochemical properties of htt aggregation both in bulk solution and at lipid interface with potential implication for HD.

In the first and second part of my $\mathrm{PhD}$ work, supported lipid bilayers comprised with curved and flat regions to demonstrate that membrane curvature plays an important role in htt aggregation were developed and validated. AFM in solution is a widely valuable technique to study morphological and mechanical properties of supported lipid bilayers, but one of the limitations associated with comparing these results to physiologically relevant membranes is the lack of membrane curvature associated with such systems. When exposed this system of bilayer to an N-terminal htt fragment, the peptide preferentially accumulated on curved regions of the bilayer. This accumulation and disruption may represent a mechanistic detail of disruption of the normal lipid associated functions of htt may play a role in toxicity associated with expanded polyQ domains. Apart from physical properties of membranes, several chemical properties can potentially play a role in regulating the interaction of htt exon1 fragments facilitated by $\mathrm{Nt}^{17}$ with lipid membranes. This took me to my third project on how acetylation modulates the binding of htt to lipid membranes. It was demonstrated that lysine residues play an important role in both aggregation and membrane binding, and this role can be affected by removal of lysine's positive charge via acetylation. The biophysical impact of this PTM could be important in htt's normal function, as well as have a modulating effect on toxicity related to mutant htt, and could lead to better defined therapeutic targets. Finally, adding small amounts of exogenous Cholesterol and GM1 to TBLE bilayers was found to be another way to slows down htt aggregation.

5. Outlook: Other relevant post translational modifications and small molecules as potential modulators of huntingtin aggregation. 
Overall, the findings presented here further underpinned the groundwork of HD research, with the eventual goal of providing insights for the development of therapeutic targets and treatment of this devastating disease. Considering the emerging impact of small molecules in neurodegenerative diseases, further studies will be required to understand the mechanistic of roles of these molecules (EGCG, curcumin, Hsc70) on htt aggregation at lipid interfaces other than bulky solution as this could have an enormous importance in HD therapeutic development.

\subsection{References}

1. Prinetti, A., et al., Glycosphingolipid behaviour in complex membranes. Biochimica et Biophysica Acta (BBA)-Biomembranes, 2009. 1788(1): p. 184193.

2. Furuse, H., et al., Effect of the mono-and tetra-sialogangliosides, GM1 and GQ1b, on long-term potentiation in the CAl hippocampal neurons of the guinea pig. Experimental brain research, 1998. 123(3): p. 307-314.

3. Maglione, V., et al., Impaired ganglioside metabolism in Huntington's disease and neuroprotective role of GM1. The Journal of Neuroscience, 2010. 30(11): p. 4072-4080.

4. Di Pardo, A., et al., Ganglioside GM1 induces phosphorylation of mutant huntingtin and restores normal motor behavior in Huntington disease mice. Proceedings of the National Academy of Sciences of the United States of America, 2012. 109(9): p. 3528-3533. potential modulators of huntingtin aggregation. 
5. Gu, X., et al., Serines 13 and 16 Are Critical Determinants of Full-Length Human Mutant Huntingtin Induced Disease Pathogenesis in HD Mice. Neuron, 2009. 64(6): p. 828-840.

6. Ehrnhoefer, D.E., L. Sutton, and M.R. Hayden, Small changes, big impact: posttranslational modifications and function of huntingtin in Huntington disease. Neuroscientist, 2011. 17(5): p. 475-92.

7. Wang, J., et al., Potential application of grape derived polyphenols in huntington's disease. Translational Neuroscience, 2010. 1(2): p. 95-100.

8. Young, F.B., et al., Putting proteins in their place: Palmitoylation in Huntington disease and other neuropsychiatric diseases. Progress in Neurobiology, 2012. 97(2): p. 220-238.

9. Hannoun, Z., et al., Post-translational modification by SUMO. Toxicology, 2010. 278(3): p. 288-293.

10. Müller, S., et al., SUMO, ubiquitin's mysterious cousin. Nature reviews Molecular cell biology, 2001. 2(3): p. 202-213.

11. Ulrich, H.D., The SUMO system: an overview, in SUMO Protocols2009, Springer. p. 3-16.

12. Kroetz, M.B., SUMO: a ubiquitin-like protein modifier. The Yale journal of biology and medicine, 2005. 78(4): p. 197.

13. Steffan, J.S., et al., SUMO modification of Huntingtin and Huntington's disease pathology. Science, 2004. 304(5667): p. 100-104.

5. Outlook: Other relevant post translational modifications and small molecules as potential modulators of huntingtin aggregation. 
14. Mukherjee, S., et al., Small ubiquitin-like modifier (SUMO) modification of the androgen receptor attenuates polyglutamine-mediated aggregation. Journal of Biological Chemistry, 2009. 284(32): p. 21296-21306.

15. O'Rourke, Jacqueline G., et al., SUMO-2 and PIAS1 Modulate Insoluble Mutant Huntingtin Protein Accumulation. Cell Reports, 2013. 4(2): p. 362-375.

16. Subramaniam, S., et al., Rhes, a Striatal Specific Protein, Mediates MutantHuntingtin Cytotoxicity. Science, 2009. 324(5932): p. 1327-1330.

17. DiFiglia, M., et al., Huntingtin is a cytoplasmic protein associated with vesicles in human and rat brain neurons. Neuron, 1995. 14(5): p. 1075-1081.

18. Sharp, A.H., et al., Widespread expression of Huntington's disease gene (IT15) protein product. Neuron, 1995. 14(5): p. 1065-1074.

19. Waelter, S., et al., The huntingtin interacting protein HIP1 is a clathrin and $\alpha$ adaptin-binding protein involved in receptor-mediated endocytosis. Human Molecular Genetics, 2001. 10(17): p. 1807-1817.

20. Suopanki, J., et al., Interaction of huntingtin fragments with brain membranes clues to early dysfunction in Huntington's disease. Journal of Neurochemistry, 2006. 96(3): p. 870-884.

21. Kegel, K.B., et al., Polyglutamine expansion in huntingtin alters its interaction with phospholipids. Journal of Neurochemistry, 2009. 110(5): p. 1585-1597.

22. Kegel, K.B., et al., Huntingtin associates with acidic phospholipids at the plasma membrane. Journal of Biological Chemistry, 2005. 280(43): p. 36464-36473.

5. Outlook: Other relevant post translational modifications and small molecules as potential modulators of huntingtin aggregation. 
23. Kegel, K.B., et al., Polyglutamine expansion in huntingtin increases its insertion into lipid bilayers. Biochemical and Biophysical Research Communications, 2009. 387(3): p. 472-475.

24. Thompson, L.M., et al., IKK phosphorylates Huntingtin and targets it for degradation by the proteasome and lysosome. Journal of Cell Biology, 2009. 187(7): p. 1083-1099.

25. Atwal, R.S., et al., Kinase inhibitors modulate huntingtin cell localization and toxicity. Nature Chemical Biology, 2011. 7(7): p. 453-460.

26. Burke, K.A., et al., The Interaction 288(21), pp.14993-15005 of Polyglutamine Peptides With Lipid Membranes is Regulated by Flanking Sequences Associated with Huntingtin. Journal of Biological Chemistry, 2013. 288(21): p.14993-15005

27. Jayaraman, M., et al., Kinetically Competing Huntingtin Aggregation Pathways Control Amyloid Polymorphism and Properties. Biochemistry, 2012. 51(13): p. 2706-2716.

28. O'Rourke, J.G., et al., SUMO-2 and PIAS1 Modulate Insoluble Mutant Huntingtin Protein Accumulation. Cell Reports, 2013. 4(2): p. 362-375.

29. Mishra, R., et al., Serine Phosphorylation Suppresses Huntingtin Amyloid Accumulation by Altering Protein Aggregation Properties. Journal of Molecular Biology, 2012. 424(1-2): p. 1-14.

30. Mishra, R., et al., Inhibiting the Nucleation of Amyloid Structure in a Huntingtin Fragment by Targeting alpha-Helix-Rich Oligomeric Intermediates. Journal of Molecular Biology, 2012. 415(5): p. 900-917.

5. Outlook: Other relevant post translational modifications and small molecules as potential modulators of huntingtin aggregation. 
31. Caron, N.S., et al., Polyglutamine domain flexibility mediates the proximity between flanking sequences in huntingtin. Proceedings of the National Academy of Sciences, 2013. 110(36): p. 14610-14615.

32. Engel, M.F.M., et al., The Polyphenol EGCG Inhibits Amyloid Formation Less Efficiently at Phospholipid Interfaces than in Bulk Solution. Journal of the American Chemical Society, 2012. 134(36): p. 14781-14788.

33. Block, R.C., et al., Altered cholesterol and fatty acid metabolism in Huntington disease. Journal of Clinical Lipidology. 4(1): p. 17-23.

5. Outlook: Other relevant post translational modifications and small molecules as potential modulators of huntingtin aggregation. 
West Virginia University Electronic

Thesis and Dissertation Signature

Form

Student Name: CHAIBVA MAXMORE

(Last) (First) (Middle)

Student ID \#: $\quad 701172422$

Non-WvU Email Account: chaibvexmaxGgmail reor

Degree:

Master's

$X$ Doctorate

Document Type:

Thesis

$X$ Dissertation

Document Title: Physicochemical modulation of huntingtin aggregation on lipid membranes: implications for Huntington's disease.

\section{Student Agreement:}

I hereby certify that, if appropriate, I have obtained and attached hereto a written permission statement from the owners of each third party copyrighted matter to be included in my thesis, dissertation, project report, or other research material, allowing distribution as specified upon deposit.

I hereby grant to West Virginia University and its agents the non-exclusive license to archive and make accessible, under the conditions selected upon deposit, my above mentioned document in whole or in part in all forms of media, now or hereafter known. I retain ownership rights as specified in the WVU copyright policy to the copyright of the abovementioned document. I also retain the right to use in future works (such as articles or books) all or part of this abovementioned document.

\section{Review and Acceptance:}

The above mentioned document has been reviewed and accepted by the student's advisory committee. The undersigned agree to abide by the statements above, and agree that this Signature Form updates any and all previous Signature Forms submitted heretofore.

Signed:

Nuadva (Student)

Committee:

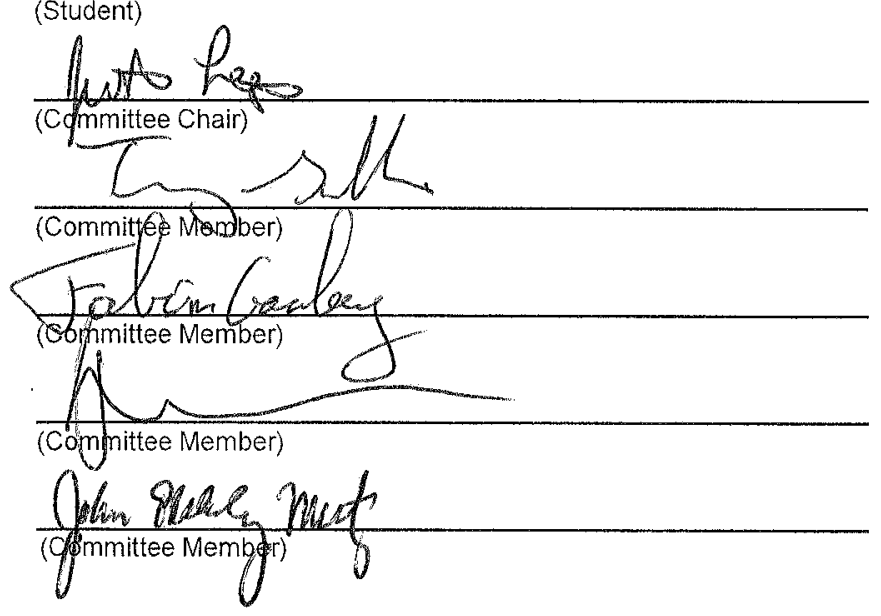

(Committee Member)

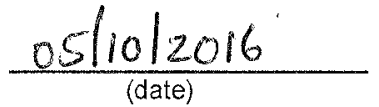

$\frac{8 / 10 / 1(\mathrm{C}}{\text { (date) }}$

$\frac{5 / 10 / 16}{\text { (date) }}$
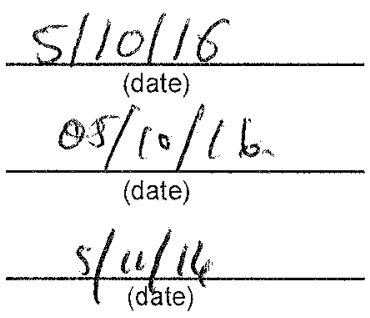

(date) 